\title{
TIP47 is recruited to lipid droplets and important for the organelle biogenesis and function
}

\author{
Dissertation \\ zur Erlangung des Doktorgrades \\ der Mathematisch-Naturwissenschaftlichen Fakultäten \\ der Georg-August-Universität zu Göttingen
}

\author{
vorgelegt von \\ Anna Valentinovna Bulankina \\ aus Rjazan/Rußland
}

Göttingen 2003 
D7

Referent:

Prof. Dr. Kurt von Figura

Korreferent:

Prof. Dr. Gerhard Braus

Tag der mündlichen Prüfung: $\quad$ 22.01.2004 
To my grandfather. 


\section{Content}

\section{Abbreviations}

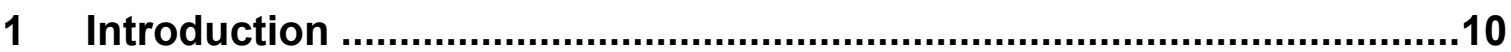

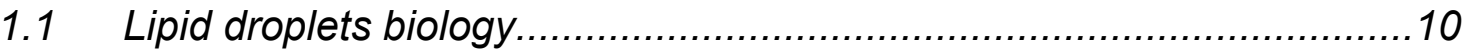

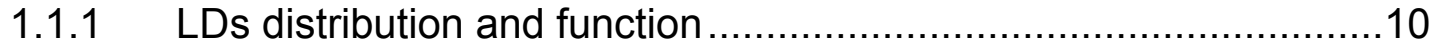

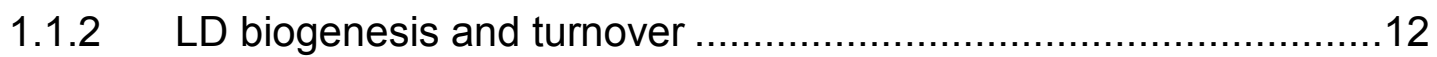

1.1.3 Lipid droplet associated proteins..............................................15

1.1.3.1 Proteins of PAT family......................................................16

1.1.3.2 Neutral lipid biosynthetic enzymes and fatty acid binding ........... proteins.................................................... 18

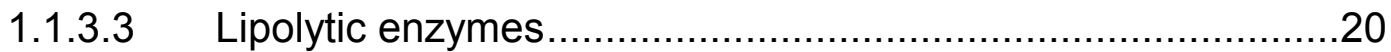

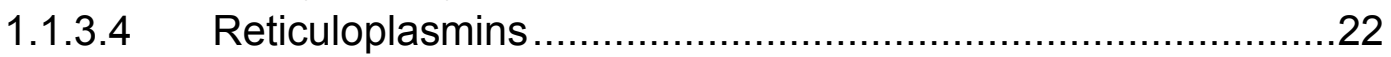

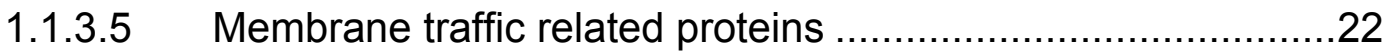

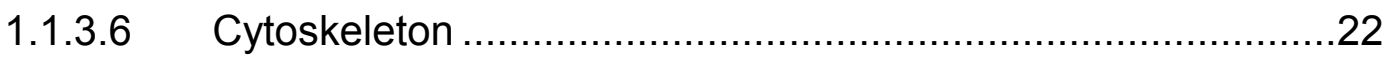

1.1.4 LDs are complex metabolically active organelles ..........................23

1.2 Transport of mannose 6-phosphate receptors and TIP47 ...................24

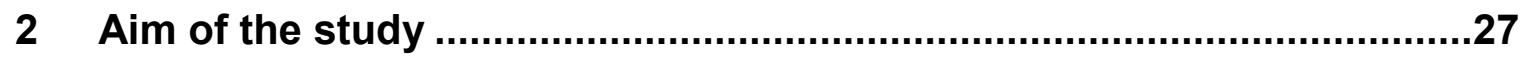

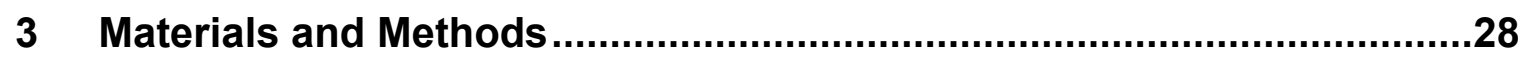

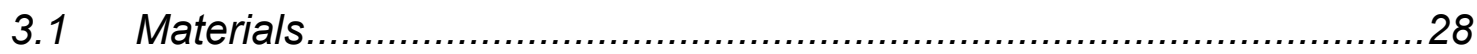

3.1.1 Equipment used for molecular biology and biochemical work ........28

3.1.2 Equipment used for microscopy ................................................

3.1.3 Materials for molecular biology and biochemical experiments .......32

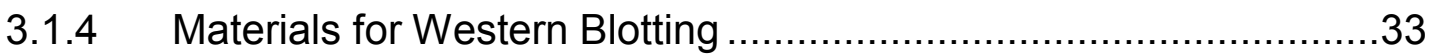

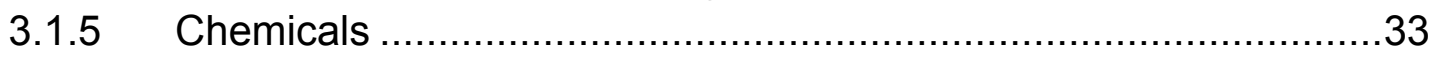

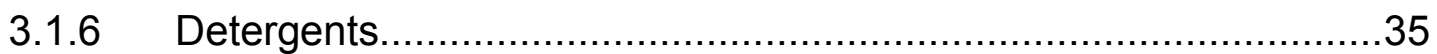

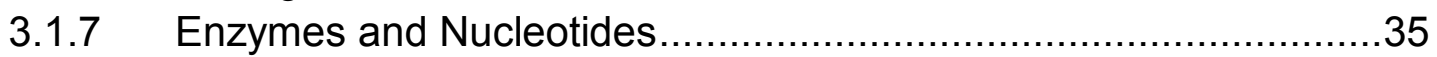

3.1.8 Proteins, Protease inhibitors and Protein standards .....................36

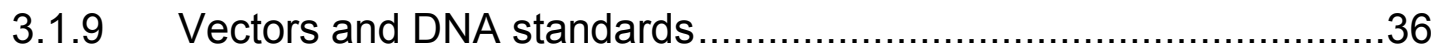

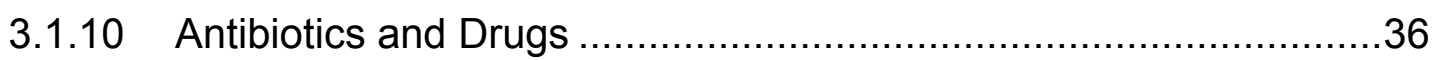

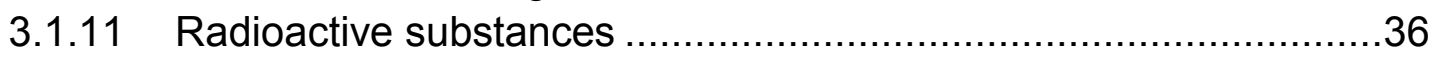

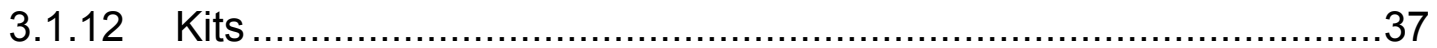

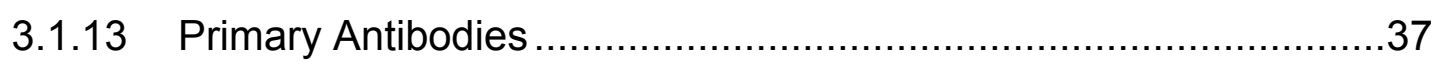

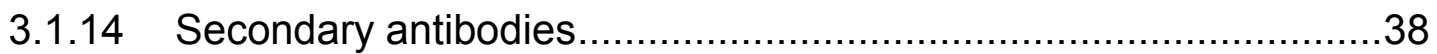

3.1.15 Bacterial strains and mammalian cell lines .................................39

3.1.16 Media and Solutions for Cell culture..........................................39

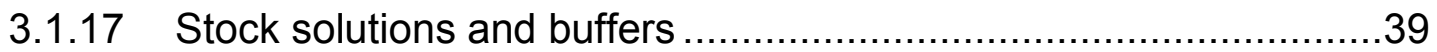

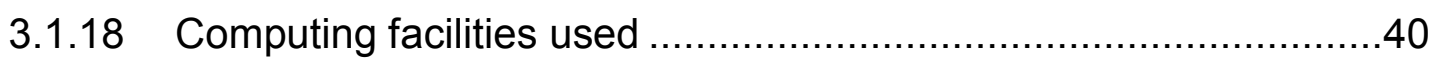


Contents

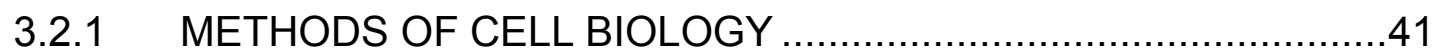

3.2.1.1 Cell Culture ................................................................. 41

3.2.1.2 Passaging of cells by trypsinization ..................................4

3.2.1.3 Freezing cells for stock maintenance ...............................42

3.2.1.4 Thawing cells from liquid nitrogen ....................................42

3.2.1.5 Transfection with Effectene transfection reagent (Quiagen) ...42

3.2.2 MOLECULAR BIOLOGY METHODS ......................................43

3.2.2.1 Cultivation of E.coli .......................................................43

3.2.2.2 Preparation of competent $E$. coli cells ...............................43

3.2.2.3 Transformation of E.coli competent cells .............................44

3.2.2.4 Preparation of electrocompetent cells ................................44

3.2.2.5 Transformation of the electrocompetent cells .......................45

3.2.2.6 Glycerol stocks of bacterial strains ...................................45

3.2.2.7 Mini preparation of plasmid DNA .......................................45

3.2.2.8 Midi preparation of plasmid DNA .....................................46

3.2.2.9 Determining the concentration of DNA ..........................47

3.2.2.10 Amplification of DNA by polymerase chain reaction (PCR) ....47

3.2.2.11 Purification of PCR products ..........................................48

3.2.2.12 Restriction endonuclease digestion of DNA .......................48

3.2.2.13 Agarose gel electrophoresis of DNA ...............................49

3.2.2.14 Extraction of DNA fragments from agarose gels ..................50

3.2.2.15 Ligation of DNA fragments ...........................................50

3.2.2.16 Cloning of full length and truncated TIP47 cDNA ..................51

3.2.2.17 Cloning of TIP47 RNAi constructs and establishing of RNAi ...... HeLa cell line .............................................................53

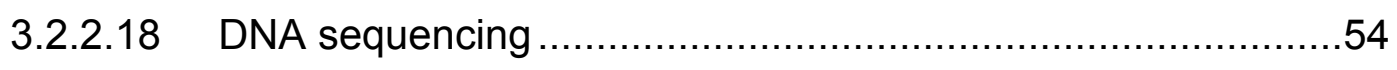

3.2.3 BIOCHEMICAL METHODS ............................................... 56

3.2.3.1 Analysis of proteins ..............................................56

3.2.3.1.1 Protein determination...............................................56

3.2.3.1.2 Solubilisation of proteins ...........................................56

3.2.3.1.3 TCA precipitation of proteins....................................56

3.2.3.1.4 SDS Poly-Acrylamide Gel Electrophoresis (SDS-PAGE).....57

3.2.3.1.5 Coomassie blue staining of Polyacrylamide gels ................58

3.2.3.1.6 Silver staining of polyacrylamide gels modified for MS ........59

3.2.3.1.7 Western blot analysis (wet method) .............................60

3.2.3.1.8 Detection technique. Chemiluminescent Substrate (PIERCE).

3.2.3.1.9 Stripping the antibody from the membrane ......................61

3.2.3.2 Metabolic labeling of cells with [35S]-Methionine/Cysteine .....61

3.2.3.3 Phosphorylation of TIP47 in vivo..................................62

3.2.3.4 Immunoprecipitation of TIP47 ….....................................63

3.2.3.5 Immunoprecipitation of MPR46 .................................64 
3.2.3.6 Isolation of lipid droplets by subcellular fractionation .65

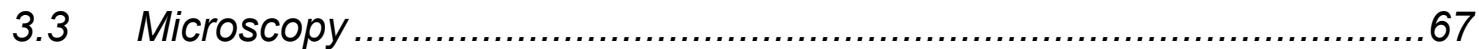

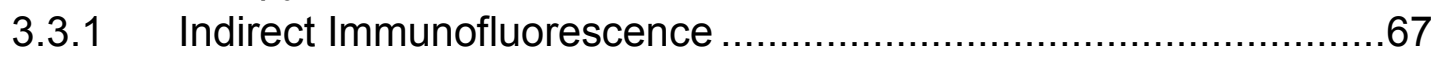

3.3.1.1.1 Fixing cells by Methanol..................................................67

3.3.1.1.2 Fixing cells by paraformaldehyde (PFA) ……………........68

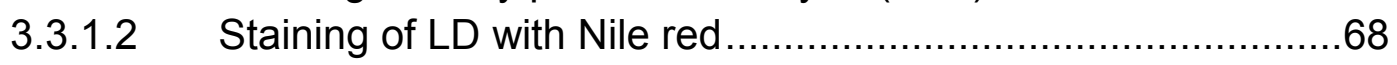

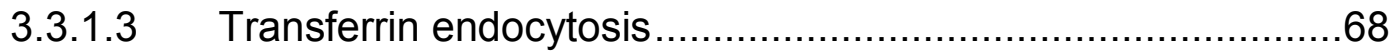

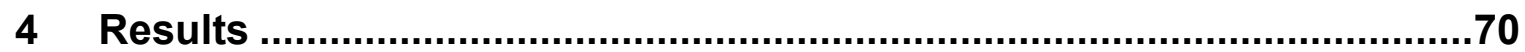

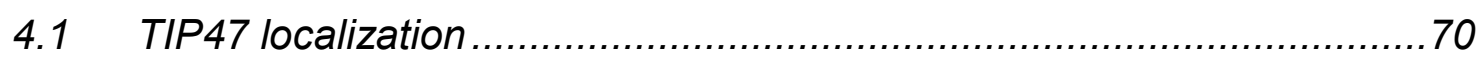

4.1.1 TIP47 detection in HeLa cells.............................................

4.1.2 Localisation of endogenous TIP47 …………........................

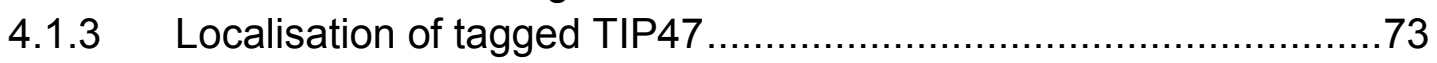

4.1.4 TIP47 cofractionates with isolated LDs ...................................75

4.1.5 TIP47 is not uniformly distributed on the LD surface......................76

4.1.6 Time course of TIP47 recruitment to the LD ..............................77

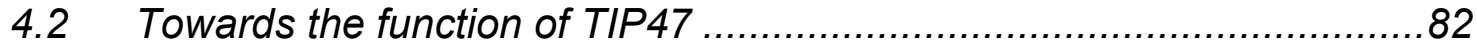

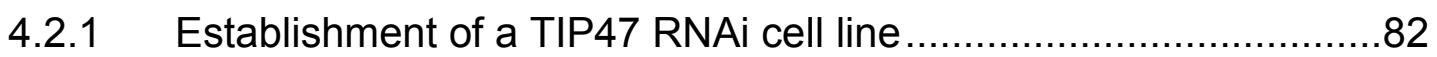

4.2.2 Characterization of TIP47 RNAi cell line ....................................84

4.2.2.1 MPRs transport is not affected by TIP47 RNAi ......................84

4.2.2.2 The effect of TIP47 RNAi on LDs........................................86

4.2.2.2.1 LD morphology is altered by TIP47 RNAi ..........................86

4.2.2.2.2 Fatty acid uptake and incorporation into LDs......................87

4.2.2.2.3 Phospholipid and protein composition of lipid droplets ........89

4.3 TIP47 secondary structure and modifications …..............................92

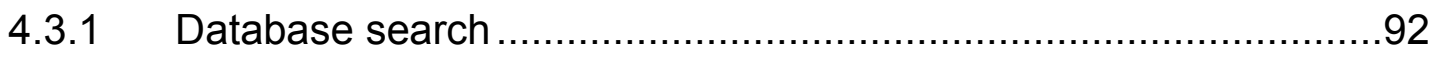

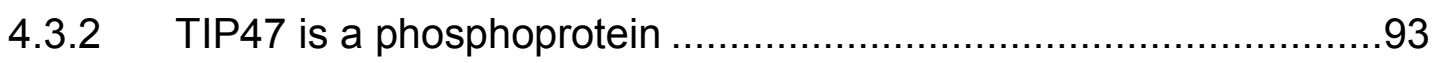

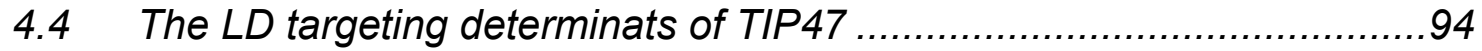

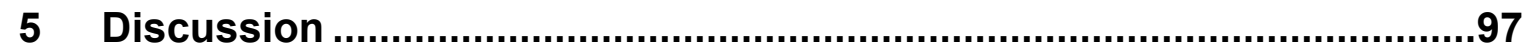

5.1 TIP47 does not participate in MPRs trafficking .................................97

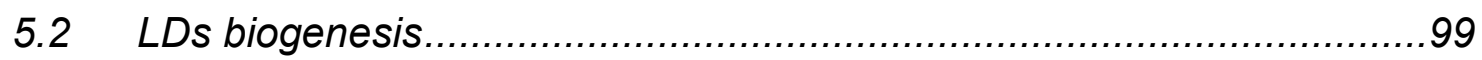

5.3 The function of TIP47 in LD biogenesis..........................................101

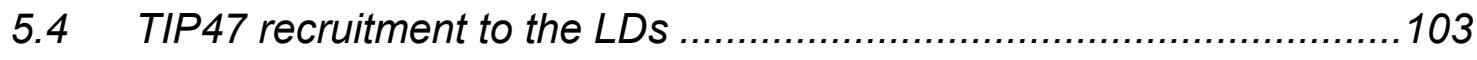

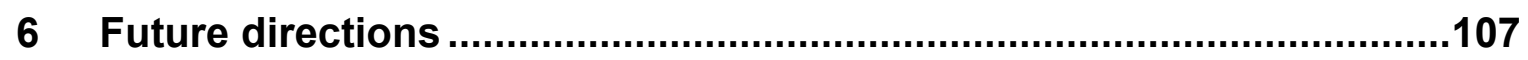

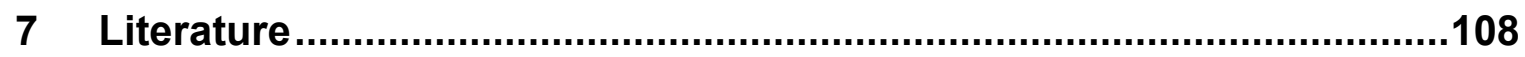




\section{Abbreviations}

ACS acyl-CoA synthetase

ADRP Adipose differentiation related protein

Amp Ampicillin

AP Adaptor Protein

APS Ammoniumperoxodisulfate

ARF Adenosine 5'-diphosphate Ribosylation Factor

BSA Bovine Serum Albumin

cAMP Adenosine 3',5'-cyclic monophosphate

cDNA complementary DNA

$\mathrm{CHO} \quad$ Chinese Hamster Ovary

$\mathrm{ddH}_{2} \mathrm{O}$ double distilled water

ddNTPs di-desoxynucleotides

DGAT Acyl-CoA-diacylglycerol acyltransferase CoA

DMEM Dulbecco's Modified Eagles Medium

DMS Dimethylsulfoxide

DNA Deoxyribonucleicacid

dNTPs Deoxynucleosidetriphosphate (dATP, dGTP, dCTP, dTTP)

dsRNA Double-stranded RNA

DTT Dithiothreitol

E. coli Escherichia coli

EDTA Ethylenediamintetraacetate-Disodium salt

ER Endoplasmic Reticulum

$\mathrm{EtOH} \quad$ Ethanol

FACL Fatty acid (long chain) CoA ligase

FABP Fatty acid binding protein

FATP Fatty acid transport protein

FCS Fetal Calf Serum

GFP Green fluorescent protein

GGA Golgi-localized Gamma ear domain-containing ARF binding proteins 


\begin{tabular}{ll}
\hline GST & Glutathione-S-Transferase \\
GTP & Guanosine 5'-triphosphate \\
IIF & Indirect immunofluorescence \\
h & Hour \\
HeLa & Henrietta Lacks \\
HEPES & N-2-Hydroxyethylpiperazin-N'-2-ethanesulfonic acid \\
HRP & Horseradish-Peroxidase \\
HSL & Hormone sensitive lipase \\
HSP & Heat shock protein \\
kb & Kilobase \\
kD & Kilodalton \\
I & Liter \\
LB & Luria Bertani \\
LD & Lipid droplet \\
M & Molar \\
mA & Milliampere \\
mg & Milligram \\
min & Minute \\
ml & Milliliter \\
mM & Millimolar \\
MPR & Mannose 6-phosphate receptor \\
MS & Mass spectrometry \\
NLSD & Neutral lipid storage disease \\
NSF & N-ethylmaleimide Sensitive Factor \\
OA & Oleic acid \\
OD & Optical density \\
PAGE & Poly-acrylamide gel electrophoresis \\
PAT family & Perilipin, ADRP and TIP47 family \\
PBS & Phosphate buffer solution \\
PCR & Polymerase chain reaction \\
PDI & Protein disulfide isomerase \\
PFA & Paraformaldehyde \\
\end{tabular}


$\mathrm{pH} \quad$ Negative logarithm of $\mathrm{H}^{+}$concentration

PKA Protein Kinase A

PM Plasma Membrane

PMSF Phenylmethylsulfonylfluoride

RNA Ribonucleicacid

RNAi RNA interference

$S \quad$ Seconds

SDS Sodium Dodecyl Sulfate

siRNA Small interfering RNA

SNARE Soluble N-ethylmaleimide Attachment protein Receptor

TAE Tris-Acetate-EDTA-Buffer

TAG Triacylglycerol

TCA Trichloroacetic acid

TE Tris-EDTA-Buffer

TEMED N,N,N',N'-Tetramethylethylenediamine

TIP47 Tail interacting protein of $47 \mathrm{kD}$

TGN trans-Golgi network

Tris Tris-(hydroxymethyl)-aminomethane

U Unit

UV Ultraviolet

V Volt

VLDL Very low density lipoprotein

$\mathrm{v} / \mathrm{v} \quad$ (volume/volume)

Vti1a Vps10p interacting

$\mathrm{w} / \mathrm{v} \quad$ (weight/volume)

wt Wild type

YFP Yellow fluorescent protein

$\alpha$-SNAP $\alpha$-soluble NSF attachment protein

$\mu \quad$ Micro 


\section{Introduction}

\subsection{Lipid droplets biology}

Lipid droplet (LD) biogenesis and turnover take place in cells of most, if not all, mammalian tissues and are an integral part of lipid metabolism. LDs are organelles of $0.05-200 \mu m$ diameter (Murphy, 2001) which accumulate lipids for both, long- and short-term storage. LDs are found in different types of organisms, ranging from yeast to humans, they share many features and consist of a core of neutral lipids, mainly triacylglycerol and cholesteryl esters as well as some other components, depending on species and cell type (reviewed in (Zweytick et al., 2000)). In contrast to other organelles, LDs are surrounded by a phospholipid monolayer (Leber et al., 1994; Tauchi-Sato et al., 2002). LDs of mammalian cells are characterized by a set of associated proteins, among which the perilipins and the adipose differentiation related protein (ADRP) or adipophilin are the best characterized.

\subsubsection{LDs distribution and function}

Adipose tissue is the biggest body store of triacylglycerols, which is an important energy source. There are two types of adipose tissue, white and brown, which have been generally regarded as having different physiological functions (triacylglycerol storage and termogenesis, respectively) (Cousin et al., 1993). However, adipose tissue is not the only place where neutral lipids are accumulated. Among other neutral lipids stores of the body are steroidogenic tissues, where lipids accumulate in LDs serve as precursors for synthesis of steroid hormones. LDs can also be found in the heart (Christiansen and Jensen, 1972) and skeletal muscles (Di Mauro et al., 1980; Hulbert et al., 1979), in hepatocytes (Chao et al., 1986), in enterocytes, in epithelial cells of the mammary gland (Ghosal et al., 1994), in several types of blood cells, such as macrophages (Dvorak et al., 1983), eosinophils and neutrophils where LDs play a key role in the arachidonic acid metabolism important for the inflammatory response (Bozza et al., 1997; Dvorak et al., 1994; Weller et al., 1999).

LDs are classically recognised as storage organelles, but their function and significance for the homeostasis of a single cell and the whole body are more complex than originally thought. In addition to the deposition of energy and precursors for the biosynthesis of membrane phospholipids, hormones and 
secondary messengers, they are also important for the maintenance of the level of free unesterified fatty acids and cholesterol to avoid their toxic effect on the physiological and intracellular levels. Indeed, aberrations in LD turnover, such as upregulation of lipolysis can lead to increased levels of circulating fatty acid and result in the development of insulin resistance associated with insulin-dependent diabetes (Saltiel and Kahn, 2001).

Other pathological processes taking place in atherosclerosis and associated with cholesterol homeostasis result first in accumulation of LDs and lipid loaded lysosomes in macrophages, in other words, in transformation of the macrophages to foam cells (Goldstein et al., 1979; Ross, 1995; Small, 1988), and secondly in their apoptotic death (Feng and Tabas, 2002; Yao and Tabas, 2001). Interestingly, intracellular unesterified arachidonic acid also may induce apoptosis (Cao et al., 2000). Furthermore, deposition of unusual fatty acid in LDs and their exclusion from membrane formation might protect the cell from their toxic effect (Dodds, 1995; Lehner and Kuksis, 1996).

These facts raise the question whether the maintenance of intracellular levels of free fatty acids and cholesterol (or other metabolites) is a housekeeping function of LDs, and, as a consequence, whether the basic machinery required for LDs biogenesis is present in all cells of the human body, tightly regulated and used under certain physiological conditions. Several studies provide evidence in favor of this idea. Firstly, all mammalian cells in culture form a number of LDs then grown in medium supplemented with sufficient amounts of fatty acids ((Murphy, 2001) and personal observation). In addition to these in vitro observations, supporting evidence may result from the analysis of lipid metabolism aberrations such as human neutral lipid storage disease (NLSD). It is known that NLSD is caused by a breakdown in the regulation of the membrane phospholipid metabolism (Igal and Coleman, 1996; Igal and Coleman, 1998) resulting in accumulation of large numbers of LDs in cells of virtually all tissues examined (Chanarin et al., 1975).

In this context it is worth to note that adipophilin, one of the two most extensively studied LD associated proteins is expressed ubiquitously (Brasaemle et al., 1997b; Heid et al., 1998), however the minimum set of proteins required for LDs biogenesis remains to be identified. 


\subsubsection{LD biogenesis and turnover}

The current model of LD biogenesis in mammalian cells was described in detail by Murphy (2001). The author proposed a constitutive cycle of LD formation and turnover, which may operate in all cells and proceeds as follows:

1) nutrients such as fatty acids are imported into the cell and transported to the ER;

2) in specific regions of the ER where triacylglycerol (or other neutral lipids) biosynthetic enzymes may be clustered, neutral lipids are synthesized and accumulate between the ER membrane leaflets;

3) Nascent LDs are released into the cytoplasm;

4) LD maturation or, in other words, their increase in size, occurs via coalescence of preexisting LDs; if remodeling of the LD content takes place, it occurs via fusion this the ER membrane;

5) Storage of the neutral lipids: in case of adipocytes, LD turnover is slowed down or blocked in favor of the storage mode by perilipins;

6) Recycling/utilization: in case of adipocytes involves hormonal stimulation.

To explain the model in more detail we will focus on processes leading to accumulation of triacylglycerol.

Unesterified long chain fatty acids used for triacylglycerol synthesis are mainly imported into the cells and derive from plasma lipoproteins, i.e. chylomicrones and very low density lipoproteins (VLDL), or fatty acids bound to serum albumin (Murphy, 2001). The mechanism of fatty acid uptake is not entirely clear yet, however, it exhibits many of the kinetic properties of a facilitated process and is assumed to be predominantly transporter mediated (Gao and Serrero, 1999; Stahl et al., 2002). At least three membrane-associated fatty acid-binding proteins were proposed to play a role in fatty acid uptake: plasma membrane fatty acid binding protein (FABPpm), fatty acid translocase (FAT) and fatty acid transport protein (FATP) (Luiken et al., 1999). According to the model proposed by Stremmel and coworkers (2001), the fist step in the fatty acid uptake is their dissociation from serum albumin, involving FABPpm or FAT. Then, fatty acids can flip-flop from the outer membrane leaflet across the bilayer and associate with caveolin-1 or the small molecular weight cytosolic FABPs, a family of tissue specific proteins involved in shuttling fatty acids to cellular 
compartments, modulating intracellular lipid metabolism, and regulating gene expression (Boord et al., 2002).

Cellular uptake of long chain fatty acids can be a subject for hormonal regulation, since it was shown that translocation of the fatty acid transport proteins 1,4 to the plasma membrane and the fatty acid uptake can be induced by insulin or inhibited by TNF- $\alpha$ in adipocytes (Stahl et al., 2002).

Metabolisation of fatty acids requires the conversion to their CoA esters, a reaction that can be performed by FATP1-5/6 (Stremmel et al., 2001) or fatty acid (long chain) CoA ligase (FACL) also known as acyl-CoA synthetase (ACS) and precedes both triacylglycerol synthesis or $\beta$-oxidation (Fujino et al., 1996).

Triacylglycerols, which are the major components of LDs in most cell types, are synthesized from acyl-CoA and a product of glucose catabolism, which can be either glycerol 3-phosphate (predominant pathway) or dihydroxyacetone phosphate (minor pathway). The minimal set of enzymes for triacylglycerol synthesis from glycerol-3phosphate would include:

1) acyl-CoA synthetase to convert fatty acids into fatty acyl-CoA esters;

2) Glycerol-3-phosphate acyltransferase, which esterifies the sn-1 position of glycerol-3-phosphate using acyl-CoA to gain lysophosphatidic acid;

3) Lysophosphatidic acid acyltransferase or 1-acyl-glycerol-3-phosphate acyltransferase to synthesize phosphatidic acid;

4) Phosphatidic acid phosphatase, which hydrolyse phosphatidic acid to yield diacylglycerol;

5) Acyl-CoA-diacylglycerol acyltransferase catalyzing the last steps in the triacylglycerol synthesis (Sorger and Daum, 2003; Vance, 1998).

The localisation of most neutral lipid synthesizing enzymes in mammalian cells remains uncertain (Buhman et al., 2001), however, usually it is assumed that triglyceride synthesis takes place in the ER (Cases et al., 1998) and it has been speculated that the enzymes may be clustered in special regions of the ER that are sites of LDs formation and release (Gibbons et al., 2000). This issue has been much better studied in yeast and resulted in a different model for LDs biogenesis (see discussion).

According to the current model for LD formation in mammals, described above, newly synthesized triacylglycerols accumulate between the leaflets of the ER membrane 
(Ostermeyer et al., 2001) and eventually bud off or stay in intimate connection with the ER. Perhaps, the origination of LDs from the ER was already visualized in live cells transfected with GFP-tagged mutant caveolin-3. Caveolins are proteins known to be associated with LDs under natural (Fujimoto et al., 2001; Liu et al., 2003) or experimental conditions, when wild type or munant forms of caveolin can be artificially enriched in the ER and redirected to the LD surface (Ostermeyer et al., 2001; Pol et al., 2001). Pol and coworkers (2001) documented the formation of structures containing a caveolin-3 mutant emerging from special regions of the ER, however, the presence of neutral lipids in the observed newly formed structures was not shown.

Indeed, LDs might originate from the ER and stay in intimate connection with it while the areas of continuity between the surface layer of LDs and the membrane leaflets of the ER were revealed by electron microscopy (Blanchette-Mackie et al., 1995) and while most of YFP- or GFP-adipophilin containing structures were found in association with the ER in live HuH-7 and Vero cells (Targett-Adams et al., 2003). In case of budding of nascent LDs from the ER, further maturation of the organelles proceeds via multiple cycles of LD fusion and substitution of their coat proteins. Events such as fusion and fission of membrane bound structures imply the presence on LD surface of proteins facilitating recognition, docking and other accompanying phenomena. In fact, during maturation of LDs in adipocytes both adipophilin and S312 covering early LDs are substituted for perilipin A (Brasaemle et al., 1997b; Wolins et al., 2003), which is known to affect the distribution of LDs and promotes their clustering required for formation of one giant LD in adipocytes (Brasaemle et al., $2000 \mathrm{~b}$ ). Beside our knowledge about ability of the perilipin A to promote LD aggregation, a first step was made to identify proteins promoting LD fusion and fission. Very recently, Liu et al., (2003) could identify multiple rab proteins (e.g. rab18), the mammalian ortholog of Sec22 and $\alpha$-SNAP on LDs isolated from $\mathrm{CHO}$ cells (Liu et al., 2003).

Fusions of LDs should result in generation of excess membrane relative to volume, which could be corrected via phospholipid hydrolysis by a phospholipase A2 (van Meer, 2001) found in association with the LD in animal (Yu et al., 1998) and plant cells (May et al., 1998).

Upon accumulation in LDs, neutral lipids are constitutively lipolysed and resynthesized what show the experiments on hepatocytes, with the estimated rate of 
turnover >1.4 pools/24h (Wiggins and Gibbons, 1992) and even in adipocytes, however, in the last the turnover cycle was slowed down (Gibbons and Wiggins, 1995) by perilipin A (see 1.1.3.1). The best studied mechanism of LD turnover is hormone induced lipolysis in adipocytes and adrenocortical cells, where treatment with adrenalin (Gibbons et al., 2000) or adrenocorticotrophic hormone (Fong and Wang, 1997), respectively, results in PKA-mediated phosphorylation of perilipin A and hormone sensitive lipase $(\mathrm{HSL})$, translocation of HSL to the LD surface, decapsulation of LDs (LD-specific capsule in more detail in 1.1.3.6) and translocation of the storage organelles to the vicinity of mitochondria (the last two steps were demonstrated in adrenocortical cells) (Fong and Wang, 1997).

In contrast to adipocytes and steroidogenic cells, in hepatocytes the overall rate of lipolysis of intracellular triacylglycerol appears to be independent of hormones (insulin and glucagon) (Wiggins and Gibbons, 1992) and involves other lipase(s), probably localised in the ER (Gibbons et al., 2000). Indeed, other lipase(s) might be involved in LD turnover, while HSL-knockout mice retain $40 \%$ of wild type levels of triacylglycerol lipase activity even in their white adipose tissue (Osuga et al., 2000; Saltiel, 2000).

In summary, the current model of LDs biogenesis in mammals implies a constitutive cycle of neutral lipid storage and turnover which may operate in all cells, their regulation on both organism and cellular levels, import of the bulk fatty acids from the extracellular environment and their primary incorporation into triacylglycerol by the ER localized enzymes.

\subsubsection{Lipid droplet associated proteins}

Numerous proteins can be found on the surface of LDs. Recently, several attempts aimed at the proteomic analysis of LD derived from yeasts (Athenstaedt et al., 1999) and mammals (Liu et al., 2003; Wu et al., 2000). Unlike most enzymes, the various structural LD associated proteins in different organisms share no obvious homology with each other. Therefore, and for clarity the following text includes the knowledge about neutral lipid biosynthetic enzymes localised on LDs from various organisms, but focus on structural LD proteins in mammals. The three major mammalian LD associated proteins are Perilipin, ADRP and IIP47 constitute the PAT family. In addition to the PAT proteins, the capsular protein $\mathrm{P}_{200}$, as well as lipotransin were 
also characterized as LD associated proteins (reviewed by (Londos et al., 1999)). However, as already mentioned above, besides structural proteins and cytoskeletal elements, various enzymes and signaling molecules can be constituents of LDs.

\subsubsection{Proteins of PAT family}

The most abundant proteins associated with LDs in mammalian cells are perilipin, ADRP and TIP47 as well as more distantly related protein S3-12.

\section{Perilipins}

Perilipins are structural LD associated proteins unique for adipose and steroidogenic cells (Blanchette-Mackie et al., 1995; Greenberg et al., 1991; Servetnick et al., 1995). Alternative splicing and polyadenilation of the perilipin mRNA yield four protein isoforms: perilipin A (57 kDa), B (46 kDa), C (38 kDa) and D (26 kDa) containing common N-terminal but distinct C-terminal regions (Lu et al., 2001). The perilipins differ in their tissue distribution, with perilipin $A$ and $B$ being expressed in both adipocytes and steroidogenic cells, while perilipin C and D are found in steroidogenic cells only.

The levels of perilipin A and $C$ are regulated post-translationally and are exclusively associated with LDs (Brasaemle et al., 1997a; Greenberg et al., 1993) where they substitute adipophilin (Brasaemle et al., 1997b) and S3-12 (Wolins et al., 2003) in the process of organelle maturation.

The function of Perilipin A as a lipolytic barrier is controlled by hormonal stimulation (Tansey et al., 2003). It is known that adipocytes and steroidogenic cells share common mechanism for lipolysis of stored neutral lipids that is mediated by protein kinase $A(P K A)$ and HSL. In the mentioned cell types, perilipins are associated with the periphery of the LDs (Blanchette-Mackie et al., 1995; Greenberg et al., 1991). Upon hormonal stimulation perilipin A is hyper-phosphorylated (Clifford et al., 2000; Egan et al., 1990; Greenberg et al., 1991) and coincides with fission of the preexisting large LDs into multiple smaller ones (Clifford et al., 2000; Souza et al., 1998). At the same time HSL translocates to the LD surface from the cytosol (Brasaemle et al., 2000a; Clifford et al., 2000; Egan et al., 1992).

The view of the perilipins as a barrier for lypolisis is further supported by experiments using ectopic expression of Perilipin A or B in fibroblasts which leads to an increase of TAG storage that is resistant to hydrolysis (Brasaemle et al., 2000b). The absence 
of the barrier in mice lacking perilipin results in a decrease in adipose mass and elevated basal lipolysis (Martinez-Botas et al., 2000; Tansey et al., 2001).

\section{Adipophilin}

Adipose differentiation related protein (ADRP or adipophilin) was first identified in mouse as a protein which mRNA level increases 50-100 fold soon after onset of adipose differentiation (Jiang et al., 1992). The murine ortholog of adipophilin is a 426 amino acid protein, localized to the LD fraction after subcellular fractionation (Wolins et al., 2001). In contrast to perilipins, adipophilin appears to be expressed ubiquitously (Brasaemle et al., 1997b; Heid et al., 1998) and its expression is transcriptionally stimulated by long chain fatty acids (Gao et al., 2000).

The function of ADRP is less well understood than that of perilipin A. First, it was proposed that ADRP may be involved in the import of fatty acids, since overexpression of ADRP in Cos-7 cells leads to an increased uptake of long chain fatty acids either saturated or unsaturated (Gao and Serrero, 1999; Gao et al., 2000). In addition, ADRP interacts with long chain fatty acids in vitro (Serrero et al., 2000). However, ADRP was found exclusively on the surface of LDs (Brasaemle et al., 1997b; Wolins et al., 2001) and does not rapidly diffuse in live cells assuming only a little turnover of ADRP at the surface of LDs after their formation (Targett-Adams et al., 2003). The last two findings suggest that the effect on the fatty acid uptake is indirect and is a kind of positive feedback.

Secondly, the ability of adipophilin to bind a cholesterol analog in vitro was interpreted as a probable participation of the protein in specific targeting of unesterified cholesterol to LDs (Frolov et al., 2000).

Alternatively, it was suggested that ADRP also may act as a lipolytic barrier around lipid droplets (Wolins et al., 2003), since overexpression of ADRP in cultured cells leads to an increase in LD size and number (Imamura et al., 2002) and to elevated fatty acid uptake (Gao and Serrero, 1999).

\section{TIP47}

The Tail interacting protein of $47 \mathrm{kD}$ (TIP47), also known as a variant of placental protein pp17b, is ubiquitously expressed (Than et al., 1998) and shares $43 \%$ of identity with adipophilin. TIP47 was mainly studied as a cargo selection device for mannose-6-phosphate receptors (Barbero et al., 2002; Carroll et al., 2001; Diaz and 
Pfeffer, 1998; Hanna et al., 2002; Krise et al., 2000; Orsel et al., 2000; Sincock et al., 2003) (described in detail in 1.2.). Nevertheless, in apparent conflict with the set of data, TIP47 was found in association with LDs (Miura et al., 2002; Than et al., 2003; Wolins et al., 2001), but was not extensively studied. In the very first article on TIP47 as a LD associated protein it was shown that TIP47 localizes to the surface of LDs by indirect immunofluorescence and subcellular fractionation (Wolins et al., 2001). The authors also demonstrated that TIP47 subcellular distribution depends on the nutritional status of cells and that the protein is redistributed to the LD concurrently with triacylglycerol accumulation (Wolins et al., 2001). However, their work was immediately opposed by the group of S.R.Pfeffer (Barbero et al., 2001) who originally characterized TIP47 as a cargo selection device. The authors attributed the discrepancy in the data obtained to cross-reactivity of the used antiserum against TIP47 and insisted that the protein is not a constituent of LDs and localizes to endosomes. Nevertheless, in the next publication on the topic it was shown that GFP-tagged TIP47 concentrates around isolated LDs (Miura et al., 2002) and, later, that endogenous TIP47 surrounds LDs using a different batch of extensively tested antibodies (Than et al., 2003).

\section{S3-12}

The protein S3-12 is also related to the PAT proteins and was first identified as a protein induced during adipocyte differentiation (Scherer et al., 1998). In fact, expression of S3-12 is restricted to adipose tissue with higher protein levels in white than in brown fat (Wolins et al., 2003). Interestingly, LD association of S3-12 depends on the nutritional status of the cell, is fully reversible and may require the incorporation of fatty acids into triacylglycerol (Wolins et al., 2003).

\subsubsection{Neutral lipid biosynthetic enzymes and fatty acid binding proteins}

Most of the proteins, found in association with LDs during proteomics studies, are, indeed, various enzymes of lipid metabolism (Athenstaedt et al., 1999; Liu et al., 2003).

In mammals, the first discovered enzymes in LDs belong to the pathway of arachidonate oxidation to eicosanoids and other oxylipid mediators. The enzymes were found on LDs mainly in cells involved in inflammatory response, such as eosinophils and neutrophils. Among the proteins are prostaglandin endoperoxide 
synthase (PGH synthase), which is the initial ratelimiting enzyme involved in oxidation of arachidonic acid to produce prostaglandins (Dvorak et al., 1994) and others, including 5-lipoxygenase (5-LOX), 15-LOX and leukotrien $\mathrm{C}_{4}$ synthase (Bozza et al., 1997; Weller et al., 1999).

Despite the identification of all the principal enzymes involved in arachidonate oxidation, most enzymes of neutral lipids, in particular triacylglycerol, biosynthetic enzymes, which could act on LDs in mammalian cells are still unknown. However, prerequisites for independent of the ER accretion of LDs exist, since, firstly, Wu et al. (2000) demonstrated that LDs isolated from both liver and mammary gland contain fatty acid binding protein (FABP), with could mediate delivery of fatty acids to the LDs (Mather, 2000). Secondly, Liu et al. (2003) found first enzymes required for triacylglycerol synthesis, namely: fatty acid (long chain) CoA ligase 3 and 4, together with 11 other enzymes of the lipid metabolism on the LDs. Our poor knowledge about LD associated triacylglycerol biosynthetic enzymes of mammals can be illustrated by the example of Acyl-CoA-diacylglycerol acyltransferase (DGAT). DGAT, which catalyzes the last reaction in triacylglycerol synthesis, has been purified from the LDs of fungi (Kamisaka et al., 1997). A DGAT, specific to LDs, was characterized in yeast (Sorger and Daum, 2002), however, the enzyme still was not found on LDs derived from mammalian cells (see Table 1.1.).

Interestingly, LDs of an oleaginous fungus, studied with respect of activities of key triacylglycerol biosynthetic enzymes, contained activities of them all (Pillai et al., 1998). Similarly, most of the enzymes were identified in yeast LDs (reviewed in (Sorger and Daum, 2003)) (see Table 1.1.).

\begin{tabular}{|l|c|c|c|}
\hline $\begin{array}{l}\text { Fatty acid transport proteins/ } \\
\text { TAG biosynthetic enzymes }\end{array}$ & $\begin{array}{c}\text { Mammalian } \\
\text { LDs }\end{array}$ & $\begin{array}{c}\text { Fungus LDs } \\
\text { enzymatic } \\
\text { activity }\end{array}$ & Yeast LDs \\
\hline Fatty acid transport protein & cFABP & & FAT1 \\
\hline Fatty acid-CoA ligase & ACS/FACL3,4 & + & FAA1,4 \\
\hline $\begin{array}{l}\text { Glycerol-3-phosphate } \\
\text { acyltransferase }\end{array}$ & - & + & SLC1 $^{\text {c }}$ \\
\hline $\begin{array}{l}\text { 1-acyl-glycerol-3-phosphate } \\
\text { acyltransferase }\end{array}$ & - & & \\
\hline
\end{tabular}




\begin{tabular}{|l|c|c|c|}
\hline Phosphatidic acid phosphatase & - & + & - \\
\hline $\begin{array}{l}\text { Acyl-CoA-diacylglycerol } \\
\text { acyltransferase }\end{array}$ & - & + & Dga1p \\
\hline
\end{tabular}

Table 1.1. Fatty acid transport proteins and enzyme of triacylglycerol biosynthesis of glycerol-3phosphate pathway found on LD in yeast and mammals.

Numerous enzymes were identified as constituents of LDs, however, some of the key enzymes of various biosynthetic pathways are still missing, others are inactive when localize to LDs, i.e. squalene epoxidase (Leber et al., 1998) and NAD(P)H steroid dehydrogenase-like protein (Ohashi et al., 2003). Earlier the presence of the biosynthetic enzymes on LDs was called to question (Murphy, 2001), however, the localization was demonstrated already in several organisms and by multiple techniques, and what is, probably, more important, LD specific triacylglycerol and cholesterol biosynthetic enzymes were identified. The enzymes, which almost exclusively localize to LD in yeast, are oxidosqualene cyclase (Milla et al., 2002) and DGAT - Dga1p (Sorger and Daum, 2002). The last makes the LDs one of the two major sites of triaclyglycerol synthesis in yeast (Sorger and Daum, 2003).

\subsubsection{Lipolytic enzymes}

As many other aspects of LD biology the exact mechanisms of LD turnover are not entirely understood yet. However, the number of publications describing LD associated lipolytic enzymes is growing. The best studied among the enzymes is a hormone sensitive lipase (HSL). HSL mediates hormone stimulated mobilization of neutral lipids stored in adipocytes and steroidogenic cells. HSL is a cytoplasmic protein, which transiently associates with LD surface after phosphorylation by PKA (Clifford et al., 2000; Egan et al., 1992). The lipase can be bound to the organelle membrane via a docking protein - lipotransin identified as structural components of adipocytes LDs (Syu and Saltiel, 1999). The HSL is responsible for at least $60 \%$ of triacylglycerol lipolysis in the white adipocytes and for all neutral cholesterol ester hydrolase activities in the testes, brown and white adipose tissue (Osuga et al., 2000). At the same time, HSL may participate in LD turnover in cardiomyocytes and pancreatic $\beta$-cells (Gibbons et al., 2000).

In addition to the well known HSL, another tissue specific enzyme was found on LDs is a neutral triacylglycerol hydrolase (TGH) present as well in microsomal fractions from liver, kidney and intestine (Lehner et al., 1999). Nevertheless, findings of the 
enzymes can not explain recycling of LDs in other cell types. The candidates for ubiquitous lipolytic enzymes associated with LD are CGI-58 and phospholipase A2. The first of them, CGI-58, which belongs to the esterase/lipase/thioesterase family of proteins, was identified as a constituent of LDs in CHO K2 cells (Liu et al., 2003). Defects in the CGI-58 gene lead to abnormal accumulation of LDs in cells of multiple tissues, that results in a neutral lipid storage disease (NLSD) (Lefevre et al., 2001). The second potentially ubiquitous enzyme detected on LDs of both plants (May et al., 1998) and animals (Yu et al., 1998) is phospholipase A2. Noll et al. (Noll et al., 2000) hypothesized that the enzyme may partially degrade the phospholipid monolayer at the onset of triacylglycerol mobilization, thus providing access for other lipases to the neutral lipid core of LD. On the other hand, phospholipase A2 may be responsible for removal of the excess phospholipids from LD surface resulting from the organelle fusions (van Meer, 2001).

In addition, a putative lipase, related to both, plant seed phospholipase A2, and yeast LD associated triacylglycerol lipiase YMR313c, was recently identified as a constituent of LDs isolated from CHO K2 cells (Liu et al., 2003). Notably, on LDs isolated from yeast three more putative lipases are present (Athenstaedt et al., 1999)

Five mammalian lipolytic enzymes mentioned above can at least transiently associate with LDs in various tissues and promote metabolisation of the stored lipids, however, it is not clear yet whether at list some of the lipolytic enzymes are always present on the surface of LDs to mediate the continuous recycling of the neutral lipids, since even $\mathrm{CGI}-58$, that is the candidate for recycling of triacylglycerol in multiple tissue cells, translocates to LD upon induction with oleic acid (Liu et al., 2003).

It is possible, that partial degradation of the phospholipid monolayer by a phospholipase as it was proposed for phospholipase A2 (Noll et al., 2000) may precede hydrolysis of neutral lipids by the other lipolytic enzymes.

The growing knowledge about constituents of LDs suggests that turnover of the organelles, in particular, hydrolysis of the stored lipids, may not require association with other metabolic organelles, e.g. the ER. 


\subsubsection{Reticuloplasmins}

Beside the various enzymes of lipid metabolism, LDs were reported to contain several ER proteins (some of them are found in the cytoplasm as well) important for proper protein folding, such as the molecular chaperones immunoglobulin binding protein (BiP) (Ghosal et al., 1994; Prattes et al., 2000), calreticulin (Ghosal et al., 1994) and calnexin (Prattes et al., 2000) as well as protein disulfide isomerase (PDI) (Ghosal et al., 1994). In a recent publication, the data were confirmed using biochemical methods on LDs isolated from $\mathrm{CHO}$ cells, which contain BiP, PDI, sec61 and heat shock protein 71 (HSP71). However, the last two proteins were regarded as contaminants (Liu et al., 2003) and it still remains to be clarified to which extend ER proteins localise to LDs or whether they are constituents of LDs at all.

\subsubsection{Membrane traffic related proteins}

LDs are membrane bound organelles, which undergo fusion and fission, however, the underlying mechanisms and proteins facilitating the processes remain obscure. It was proposed that the cytoskeleton could control LD dynamic (Murphy, 2001), but the idea was not entirely developed yet. In the most recent publication, Liu et al. (2003) could identify several proteins known to be involved in membrane traffic associated with LD, in particular, nine rab proteins (e.g. rab18), which are the small GTP-ases regulating docking and fusion of membrane-bound organelles (Soldati et al., 1995), the mammalian ortholog of Sec22, one of the ER resident SNARE proteins (soluble NSF attachment protein receptor) (Hay et al., 1996), a-SNAP (a-soluble NSF attachment protein), which functions in dissociation of the SNARE complexes (Marz et al., 2003), as well as RalA, a small GTPase regulating, among other processes, membrane traffic in polarized cells (Moskalenko et al., 2002).

In case these findings will be confirmed, that would support the idea that membrane trafficking of organelles bound with a single or double leaflet membrane share common features.

\subsubsection{Cytoskeleton}

The association of LDs with the cytoskeleton was demonstrated by means of light and electron microscopy (Franke et al., 1987; Targett-Adams et al., 2003; Wang et al., 1997) and was also partially biochemically characterized. Up to now the most remarkable LD-associated cytoskeletal structures are regular vimentin cages formed during adipose conversion of 3T3-L1 cells (Franke et al., 1987; Lieber and Evans, 
1996; Wang et al., 1997). In contrast to adipose, in steroidogenic cells LDs are randomly attached to vimentin filaments (Almahbobi and Hall, 1990; Almahbobi et al., 1992). Despite the different organisation of vimentin cytoskeleton, in both cell types LDs are associated with a capsular protein $\mathrm{P}_{200}$ (Fong and Wang, 1997; Fong et al., 1996; Wang and Fong, 1995; Wang et al., 1997), which was supposed to function as a linker between the LD membrane and vimentin filaments. Indeed, $P_{200}$ could promote rearrangement of vimentin, since $P_{200}$ translocation to the nascent LDs follows the adipose conversion of preadipocytes and precedes the vimentin cage formation (Lieber and Evans, 1996; Wang et al., 1997). Moreover, $P_{200}$ may serve as a regulator of the association with cytoskeletal elements, since it detaches from LDs and translocates to the cytosol upon hormonal stimulation of steroid secretion (Fong et al., 2002; Wang and Fong, 1995).

LDs in other cells types are not surrounded with vimentin cages or $\mathrm{P}_{200}$, but may interact with microtubules and/or actin cytoskeleton. The transport of LDs from the basal region to the apical membrane of mammary epithelial cells is supposed to be necessary for milk lipid globule secretion (Wu et al., 2000). Attempts to elucidate the components of the transport machinery associated with LDs also revealed the presence of at least four potentially important proteins, including dynein intermediate chain (facilitating interaction of cargo with microtubules), gelsolin (implicated in actin remodeling), gephyrin (involved in membrane protein-cytoskeleton interaction) and motor protein (of unknown function) (Wu et al., 2000).

The vectorial transport (most likely along microtubules) of a minor fraction of LDs was visualized in HuH-7 cells (Targett-Adams et al., 2003).

Thus, LDs could be integrated into the intracellular environment by means of various cytoskeletal elements: vimentin, actin and microtubules, in a tissue specific manner.

\subsubsection{LDs are complex metabolically active organelles}

There is growing evidence in favour of the idea that LDs are complex organelles directly participating in both lipid metabolism and intracellular signalling since they possess multiple proteins which could be potentially involved in the processes. Moreover, the most recent publications (Athenstaedt et al., 1999; Liu et al., 2003; Sorger and Daum, 2003), describing LD associated triacylglycerol biosynthetic enzymes and lipases, support the hypothesis that LDs have the potential of independent from other organelles neutral lipids biosynthesis and turnover. 


\subsection{Transport of mannose 6-phosphate receptors and TIP47}

Vesicle-mediated transport is a process required to establish and to maintain the unique composition of eukaryotic membranous organelles. Organelles of the secretory and endocytic pathways such as the endoplasmic reticulum (ER), the Golgi apparatus (GA), the trans-Golgi network (TGN), as well as endosomes and lysosomes continuously exchange material with each other. The process includes formation of a transport intermediate or a vesicle and its fusion with an acceptor compartment. Selection of cargo proteins into a newly forming vesicle requires a sorting event during which a specific interaction between the cargo proteins and cargo selection devices or adaptor molecules which are part of the transport machinery occurs. This machinery includes scaffolding proteins, such as clathrin, multiple GTPases, regulating docking and fusion of membrane-bound organelles, motor proteins, such as dynamin, SNAREs and other proteins promoting docking and fusion with an acceptor membrane.

Two of the model cargo proteins whose transport was intensively studied so far are the mannose 6-phosphate receptors (MPRs). The MPRs are transmembrane proteins continuously recycling in the secretory and endocytic pathways (see Figure 1.1.). The function of the receptors is binding and delivery of soluble lysosomal enzymes to late endosomes. There are two MPRs different in molecular weight and calcium dependency called MPR46 (calcium-dependent) and MPR300 (calciumindependent). Both receptors have similar transport routes in the cell. 


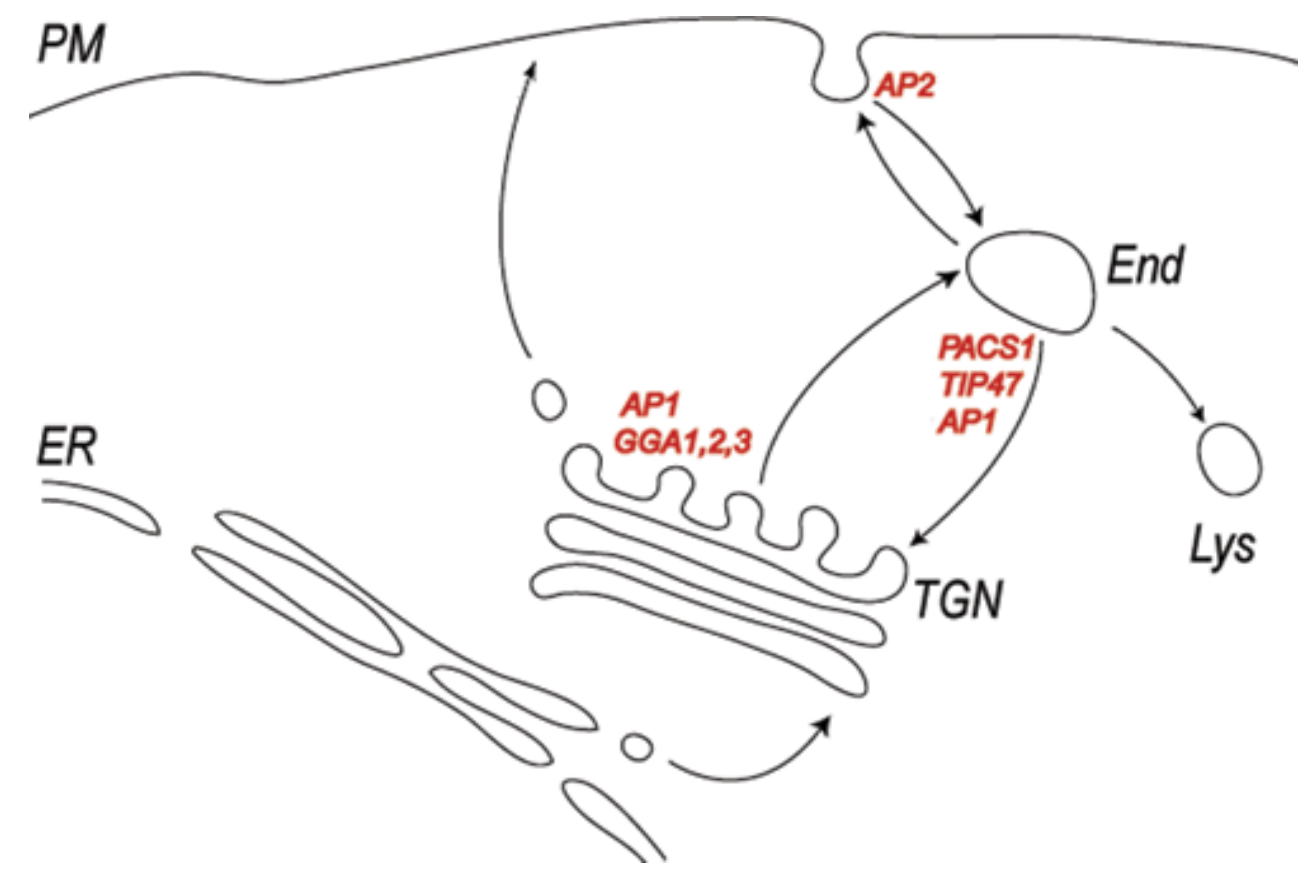

Figure 1.1. Transport route of MPRs in the cells. Newly synthesized MPRs are transported from the ER through the Golgi stack to the TGN where they are recruited into clathrin coated pits by means of adaptor complex AP1 and GGA1, 2, 3 proteins and delivered to an endosomal compartment (End). Alternatively, MPRs escape to the plasma membrane (PM) and are internalized from the cells surface by means of adaptor complex AP2 (clathrin coated vesicles). From the endosomes MPRs are either retrieved to the TGN (candidate cargo selection devices are AP1,3, PACS1 or TIP47) or finally degrade in the lysosomes (Lys).

Adaptors involved in MPRs transport from various organelles are AP1 and GGAs (Golgi-localizing, gamma-adaptin ear homology domain, ARF-binding proteins) at the TGN and AP2 at the plasma membrane. AP1 (Mallard et al., 1998; Meyer et al., 2000), AP3 (Medigeshi and Schu, 2003; Storch and Braulke, 2001), PACS1 (Wan et al., 1998) and TIP47 (Diaz and Pfeffer, 1998) are other candidates which could determine the retrieval of MPRs from an endosomal compartment to the TGN.

The view on TIP47 as a cargo selection device was based on a row of evidences, which seems to be clear. TIP47 was initially identified in a yeast two-hybrid system as a protein interacting with MPRs cytoplasmic domains (Diaz and Pfeffer, 1998). Further analysis of the protein revealed that it binds MPRs cytoplasmic domains expressed and purified as GST fusion proteins and colocalizes with MPR300 in endosomes (Diaz and Pfeffer, 1998). Moreover, the authors claimed that TIP47 association with membranous organelles requires the presence of MPRs and is GTP. ase stimulated. In the same work TIP47 was found to be important for recycling of MPRs from the endosomes to the TGN in an in vitro transport assay and in live cells, where cytosolic amounts of TIP47 were decreased by antisense oligonucleotides. Later, the interactions of TIP47, MPR46, MPR300 and the small GTP-ase, which 
turned out to be rab9, were studied in detail (Carroll et al., 2001; Diaz and Pfeffer, 1998; Hanna et al., 2002; Krise et al., 2000; Orsel et al., 2000; Sincock et al., 2003). Summarizing, S.R.Pfeffer and colleagues postulated that TIP47 localizes on endosomes and is required for MPRs recycling in vivo and in vitro and that the sorting event is facilitated by a small GTP-ase rab9. 


\section{Aim of the study}

The trafficking of the mannose 6-phosphate receptors (MPRs) has been studied for many years, but still some important details remain controversial. For example, it is still an ongoing debate how MPRs are sorted for recycling from endosomes to the TGN. Several adaptor molecules have been postulated to play an important role in the process including AP1 and TIP47. Mainly due to the work of Suzanne Pfeffer and colleagues, TIP47 was characterized as a cargo selection device for MPRs, determining their transport from endosomes back to the TGN. However, several other groups recently found TIP47 associated with the surface of lipid droplets and suggested that TIP47 functions in lipid metabolism. Nevertheless, a direct functional proof for this concept is still missing. Lipid droplets are peculiar storage organelles and supposed not to have any relationship with MPR trafficking pathways. Due to these uncertainties about the functional role of TIP47, the aim of this study was:

1. to revise the intracellular TIP47 localization and its recruitment to membranes using endogenous protein as well as cells expressing recombinant TIP47.

2. to establish a cell line not expressing TIP47 by a plasmid based RNA interference technique.

3. to study MPR46 distribution and recycling in such TIP47 deficient cells.

4. to analyze the putative function of TIP47 in lipid metabolism/lipid droplet formation. 


\section{$3 \quad$ Materials and Methods}

\subsection{Materials}

\subsubsection{Equipment used for molecular biology and biochemical work}

\begin{tabular}{|c|c|}
\hline $\begin{array}{l}\text { Analytical weighing balance } \\
\text { Type CP225D \& M5P }\end{array}$ & Sartorius, Göttingen \\
\hline Autoclave Type Tecnoclav 50 & $\begin{array}{l}\text { Tecnorama, Zürich, } \\
\text { Switzerland }\end{array}$ \\
\hline $\begin{array}{l}\text { Balances } \\
\text { Type BP3100P \& CP3202P }\end{array}$ & Sartorius, Göttingen \\
\hline $\begin{array}{l}\text { Chamber for PAGE and wet } \\
\text { Western blotting Mini-V } 8 \times 10\end{array}$ & Gibco BRL Life Technologies, Inc \\
\hline ELISA reader, TECAN SPECTRA & SLT Instruments, Germany \\
\hline $\begin{array}{l}\text { Electrophoresis Power Supplies: } \\
\text { Biometra P25 \& PP4000 } \\
\text { St } 305\end{array}$ & $\begin{array}{l}\text { Schüt Labortechnik, Göttingen } \\
\text { Gibco BRL Life Technologies, Inc }\end{array}$ \\
\hline Electroporator 1000 Stratagene $\AA$ & Cortland NY, USA \\
\hline Refrigeretor $+4^{\circ} \mathrm{C}$ & Liebherr \\
\hline Freezer $-20^{\circ} \mathrm{C}$ & Liebherr \\
\hline$-85^{\circ} \mathrm{C}$ Ultra Low Freezer & $\begin{array}{l}\text { New Brunswick Scientific Co, Inc., } \\
\text { Edison, USA }\end{array}$ \\
\hline Gel documentation system & Hitachi, Japan \\
\hline GelAir Dryer & Bio-Rad, USA \\
\hline Heating block 5320 \& 5436 & Eppendorf, Hamburg \\
\hline Ice Machine & Ziegra, Isernhagen \\
\hline $\begin{array}{l}\text { Incubator for bacterial liquid } \\
\text { cultures }\end{array}$ & $\begin{array}{l}\text { New Brunswick Scientific Co, Inc., } \\
\text { Edison, USA }\end{array}$ \\
\hline Incubator for bacterial plates & Heraeus, Osterode \\
\hline $\begin{array}{l}\text { Incubators for mammalian cells: } \\
\text { Infrared } \mathrm{CO}_{2} \text { incubator Labotec }\end{array}$ & Labotect GmbH, Göttingen \\
\hline
\end{tabular}




\begin{tabular}{|c|c|}
\hline STERI:CULT incubator & Forma Scientific, Ohio \\
\hline $\begin{array}{l}\text { Liquid Nitrogen tanks } \\
\text { Biosafe Chronos }\end{array}$ & $\begin{array}{l}\text { Messer Griesheim, } \\
\text { Frankfurt/M }\end{array}$ \\
\hline $\begin{array}{l}\text { Liquid Scintillation Counter } \\
\text { LS } 6500\end{array}$ & Beckman, USA \\
\hline $\begin{array}{l}\text { Luminescent Image Analyzer } \\
\text { Fujifilm LAS-1000 plus }\end{array}$ & Fuji photo film Co. Ltd., Japan \\
\hline $\begin{array}{l}\text { Magnetic stirrers: } \\
\text { Ikamag Ret } \\
\text { MR3000 }\end{array}$ & $\begin{array}{l}\text { Janke \& Kunkel, Staufen } \\
\text { Heidolph, Germany }\end{array}$ \\
\hline Microflow Biological Safety Cabinet & Nunc, Wiesbaden \\
\hline SterilGARD Hood & $\begin{array}{l}\text { the BAKER COMPANY, inc } \\
\text { Sanford, Maine }\end{array}$ \\
\hline Microwave Oven & Siemens, Germany \\
\hline $\begin{array}{l}\text { pH-Meters: } \\
\text { Knick } \\
\text { ino Lab pH Level } 1\end{array}$ & $\begin{array}{l}\text { Schütt, Göttingen } \\
\text { WTW, Weilheim }\end{array}$ \\
\hline Phosphoimager Fujix BAS1000 & Fuji, Tokyo, Japan \\
\hline Pipet-aid & Drummond Scientific Corporation, USA \\
\hline $\begin{array}{l}\text { RNA/DNA Calculator } \\
\text { Genequant II }\end{array}$ & $\begin{array}{l}\text { Pharmacia Biotech } \\
\text { Cambridge, England }\end{array}$ \\
\hline Rocker & Institute workshop \\
\hline Sonicators: & \\
\hline Sonicator $^{\mathrm{TM}} \mathrm{W}-200 \mathrm{~F}$ & $\begin{array}{l}\text { Heat systems-Ultrasonics, INC } \\
\text { New York, USA }\end{array}$ \\
\hline Sonifier 450 & $\begin{array}{l}\text { Branson Ultrasonic SA, } \\
\text { Carouge Geneva/ USA }\end{array}$ \\
\hline Spectrophotometer, Uvikon 932 & Kontron Instruments, Italy \\
\hline Speed Vac Concentrator & BACHOFER, Germany \\
\hline Spinning Wheel & Institute workshop \\
\hline
\end{tabular}




\begin{tabular}{|l|l|}
\hline $\begin{array}{l}\text { Thermocycler } \\
\text { Mastercycler Gradient }\end{array}$ & Eppendorf, Hamburg \\
\hline Transilluminator IL-400-M & Bachofer, Reutlingen \\
\hline UV-hand lamp & Bachofer, Reutlingen \\
$(254 \mathrm{~nm}$ and 312nm) & \\
\hline Vortex Genie 2 & Bender \& Hobein AG \\
Vortex Genie 2 & Zurich, Switzerland \\
\hline Water bath Julabo U3 & Julabo Labortechnik GMBL \\
\hline Water bath shaker Köttermann & Ernst Schütt Jr, Göttingen \\
\hline
\end{tabular}

\section{Centrifuges}

\begin{tabular}{|l|l|}
\hline $\begin{array}{l}\text { Labofuge GL } \\
\text { Megafuge 1.0 }\end{array}$ & Heraeus Sepatech, Osterode/ Harz \\
\hline $\begin{array}{l}\text { Eppendorf centrifuges: } \\
\text { Microprep centrifuge } \\
\text { StartSpin } \mu \text { Prep }\end{array}$ & Eppendorf, Hamburg \\
\hline $\begin{array}{l}\text { Refrigerated centrifuge J2-MC } \\
\text { Ultracentrifuge L-80 } \\
\text { Ultracentrifuge L8-70M }\end{array}$ & Schütt Labortechnik, Göttingen \\
\hline $\begin{array}{l}\text { Rotors for cooling centrifuges and } \\
\text { ultracentrifuge: } \\
\text { (g values are for } r_{\text {max }} \text { ) }\end{array}$ & \\
\hline JA-10 rotor, upto 17,680 g & Beckman, München \\
\hline JA-20 rotor, upto 48,300 g & Beckman, München \\
\hline SW41Ti rotor, upto 210,053g & Beckman, München \\
\hline
\end{tabular}




\subsubsection{Equipment used for microscopy}

\begin{tabular}{|c|c|}
\hline $\begin{array}{l}\text { Flourescence Microscope } \\
\text { Axiovert } 100\end{array}$ & \multirow{3}{*}{ Zeiss; Oberkochen } \\
\hline $\begin{array}{l}\text { Phase-contrast microscope } \\
\text { Model ID } 03\end{array}$ & \\
\hline $\begin{array}{l}\text { Laser Scanning Microscope } \\
\text { LSM2 with software } 3.95\end{array}$ & \\
\hline $\begin{array}{l}\text { Objectives for the LSM and } \\
\text { Axiovert } 100 \\
\text { Plan-Neofluar } 25 \times / 0,81 \mathrm{~mm} \\
\text { Plan-Neofluar } 40 \times / 1,3 \mathrm{~mm} \\
\text { Plan-Neofluar } 63 \times / 1,25 \mathrm{~mm} \\
\text { Plan-Neofluar } 100 \times / 1,3 \mathrm{~mm} \\
\text { Plan Apochromat } 63 \times / 1,4 \mathrm{~mm}\end{array}$ & Zeiss; Oberkochen \\
\hline $\begin{array}{l}\text { Leica TCS SP2 AOBS } \\
\text { Filter-free microscope }\end{array}$ & $\begin{array}{l}\text { Leica Microsystems Heidelberg } \mathrm{GmbH} \text {, } \\
\text { Germany }\end{array}$ \\
\hline $\begin{array}{l}\text { Objectives for Leica DM IRE2 } \\
\text { HC PL APO 20x/0.70 } \\
\text { HCX PL APO 40x/1.25 } \\
\text { HCX PL APO63x/1.40-0.60 }\end{array}$ & $\begin{array}{l}\text { Leica Microsystems Heidelberg GmbH, } \\
\text { Germany }\end{array}$ \\
\hline $\begin{array}{l}\text { Olympus IX50 } \\
\text { Fluorescence microscope }\end{array}$ & Olympus Optical Co., LTD Japan \\
\hline $\begin{array}{l}\text { Objectives for the Olympus IX50 } \\
\text { UPlan Apo 40x/1,00mm } \\
\text { Plan Apo } 60 x / 1,40 \mathrm{~mm} \\
\text { UPlan Apo } 100 x / 1,35 \mathrm{~mm}\end{array}$ & Olympus Optical Co., LTD \\
\hline Olympus TH4-200 & Olympus Optical Co., LTD \\
\hline $\begin{array}{l}\text { CCD Camera Imago } 1 / 2 \text { " chip with } \\
640 \times 480 \text { pixels }\end{array}$ & TILL Photonics GmbH \\
\hline Monochromator Polychrome II & TILL Photonics GmbH \\
\hline
\end{tabular}




\subsubsection{Materials for molecular biology and biochemical experiments}

Autoclave bags

Bottle top filters

Cell Culture single use pipettes $5 \mathrm{ml} \& 10 \mathrm{ml}$

Cell Culture plates:

4 well NUNCLON

6 well, 24 well \& 96well CELLSTAR

Centrifuge tubes:

JA-10 Polypropylene tube

JA-20 Polypropylene tube

14x89mm Polyallomer tube

Culture dishes for mammalian cells

3; $5 ; 6 ; 10 \& 15 \mathrm{~cm} \mathrm{\varnothing}$

Culture flasks for bacteria

Culture flasks for mammalian cells

25 and $75 \mathrm{~cm}^{2}$

Dialysis bags (12,000 Da cut-off)

Delta $\mathrm{T}$ dishes $0.15 \mathrm{~mm}$

Glass pipettes

Immersion oil 518C

Microscope slides

Needles for syringes

Nunc cryotube ${ }^{T M}$ vials

Parafilm

Pasteur pipettes

Pipette tips

Plastic tubes 10, 15 and $50 \mathrm{ml}$

Polycarbonate ultracentrifuge tubes $1,5 \mathrm{ml}$
Sarstedt, Nümbrecht

Sarstedt Inc., Newton, USA

Greiner, Nürtingen

Nalge Nunc International

Denmark

Greiner bio-one, Frickenhausen

Nalgene, München

Nalgene, München

Beckman Instruments, Inc., USA

Greiner bio-one, Frickenhausen

Schott, Mainz

Greiner bio-one, Frickenhausen

Biomol, Hamburg

Bioptechs, Inc., USA

Schütt, Göttingen

Zeiss, Oberkochen

Menzel-Glaser

B.Braun Melsungen AG,

Melsungen

Nalge Nunc International

Denmark

American National Can ${ }^{\mathrm{TM}}$

Chicago

Schütt, Göttingen

Sarstedt, Nümbrecht

Sarstedt, Nümbrecht

Beckman, München 
Reaction tubes:

$0.2,0.5,1.5 \& 2 \mathrm{ml}$

Sarstedt, Nümbrecht

Scintillation Cocktail LUMASAFE PLUS

Lumac LSC B.v., The Netherlands

Scintillation Vials MINIS 2000

Zinsser Analytic, Germany

Sterile filters $0.2 \mu \mathrm{M}$

Heinemann Labortechnik GmbH

Syringes:

$1 \mathrm{ml}$ and $2 \mathrm{ml}$

B.Braun Melsungen AG, Melsungen

$5,10 \& 20 \mathrm{ml}$ Ecoject

Dispomed Witt OHG, Gelnhausen

\subsubsection{Materials for Western Blotting}

Nitrocellulose membrane $0.2 \mu \mathrm{M}$

Schleicher and Schüll, Dassel

Whatman GB002 paper

Schleicher and Schüll, Dassel

Whatman GB003 paper extra thick

Schleicher and Schüll, Dassel

Supersignal Chemiluminiscent Substrtate

Pierce, Illinois/ USA

\subsubsection{Chemicals}

Acetic acid

Roth

Acetone

Merck

Acrylamide/ Bisacrylamide (30\%/ 0.8\%)

Roth

Agar

Roth

Agarose

Roth

Ammonium chloride

Sigma

Ammonium peroxide disulphate (APS)

Merck

Bacto tryptone

Roth

Bio-Gel P-6 DG (Desalting gel)

Bio-rad

Bromophenol blue

Merck

Calcium chloride

Merck

Calcium acetate

Sigma

Chloroform

Merck

Coomassie, Serva Blue $R$

Serva

DAKO (Fluorescent mounting medium)

Dako Corporation 
Dimethylsulfoxide (DMSO)

Merck

Disodiumhydrogenphosphate

Merck

Dipotassiumhydrogenphosphate

Merck

Dithiothreitol (DTT)

Diagnostic Chemicals Ltd

Ethanol

Roth

Ethidium bromide

Serva

Ethylenediamine tetra acetic acid

-sodium salt (EDTA)

Merck

Glucose

Merck

Glutathione

Amersham Pharmacia

Glycerol

Roth

Glycine

Roth

HEPES (N-2-Hydroxyethylpiperazin-N'-

2-ethanesulfonic acid)

Roth

Isobutanol

Merck

Isopropyl alcohol

Merck

Liquid Nitrogen

Messer Griesheim, Seigen

Magnesium chloride

Merck

Manganese chloride

Merck

2-Mercaptoethanol

Merck

Methanol

Roth

MOPS

(3-[N-Morpholino]propanesulfonic acid)

Serva

Nile Red

Sigma

Paraformaldehyde (PFA)

Sigma

Ponceau S $(0.2 \%$ solution in $3 \%$ TCA)

Serva

Potassium chloride

Merck

Potassium hydroxide

Merck

Oleic acid

Sigma

Rubidium chloride

Merck

Silver nitrate

Sigma

Sodium acetate

Merck 
Sodium azide

Sodium bicarbonate

Sodium chloride

Sodium dihydrogenphosphate

Sodium hydroxide

Sodium thiosulfate

Sucrose

TEMED

(N'N'N'N Tetramethylethylenediamine)

Trichloroacetic acid

Triethanolamine

Tris-base

Yeast extract

All the chemicals were of analysis grade.

\subsubsection{Detergents}

NP -40

Saponin

Sodium deoxycholate

Sodium dodecyl sulphate (SDS)

Triton X-100

Tween-20
Sigma

Merck

Roth

Merck

Merck

Sigma

Merck

Serva

Merck

Merck

ICN Biomedicals, Aurora/ USA

Roth
Serva

Sigma

Serva

Sigma

Serva

Serva

\subsubsection{Enzymes and Nucleotides}

Restriction endonucleases

T4 DNA ligase

Pfu Ultra ${ }^{\mathrm{TM}}$ DNA Polymerase

Taq DNA polymerase

Ultra pure dNTP set
New England Biolabs

New England Biolabs

Stratagene

Pharmacia, Freiburg

Pharmacia, Freiburg 


\subsubsection{Proteins, Protease inhibitors and Protein standards}

Aprotinin

Bovine Serum Albumin (BSA)

Glutathione-sepharose

Leupeptin

PANSORBIN cells, Standardized

(Heat inactivated Staphylococcus aureus

cell suspension)

Pepstatin A

Phenylmethylsulfonylfluoride (PMSF)

Prestained protein molecular

weight standards

Protein A agarose

Protein G sepharose

\subsubsection{Vectors and DNA standards}

pGK Hygro

PMPSV-EH

$\mathrm{pSHH}$

DNA ladder
Biomol

Serva

Amersham-Pharmacia

Biomol

Calbiochem

Biomol

Serva

Biorad

Sigma

Sigma

\subsubsection{Antibiotics and Drugs}

Ampicillin

Serva

Kanamycin

Neomycin (Gentamycin sulfate or G418)

Gibco

Penicillin/Streptomycin

Gibco

$(100 x=10,000 \mathrm{U} / \mathrm{ml})$

\subsubsection{Radioactive substances}

L-[35S]-Methionin/Cystein, aqueous solution $14 \mathrm{mCi} / \mathrm{ml}$

Purchased from Amersham Pharmacia Biotech, UK.

$[\mathrm{Y}-32 \mathrm{P}]-\mathrm{ATP} 10 \mathrm{mCi} / \mathrm{ml}$

Purchased from Hartmann Analytic $\mathrm{GmbH}$, Germany

[1-14C]-Arachidonic acid, 0.050mCi/ml 
[1-14 C]-Oleic acid, $0.1 \mathrm{mCi} / \mathrm{ml}$

[9,10-3 $\mathrm{H}]$-Palmitic acid, $1.0 \mathrm{mCi} / \mathrm{ml}$

Purchased from Moravek Biochemicals Inc, USA

\subsubsection{Kits}

ABI PRISM $\otimes$ Rhodamine Terminator

Cycle sequencing Ready Reaction Kit

Bio-Rad Protein Assay

Effectene Transfection kit

GeneSilencer ${ }^{\mathrm{TM}}$ System

HiSpeed Plasmid Midi kit

Invisorb Spin Plasmid Mini kit

QIAquick Gel extraction kit

QuikChange $^{\mathrm{TM}}$ Site-Directed mutagenesis kit
Applied Biosystems

Perkin Elmer Applied Biosystems

Bio-Rad

Qiagen

IMGENEX Corporation

Qiagen

Invitek, Berlin

Qiagen

\subsubsection{Primary Antibodies}

\begin{tabular}{|c|c|c|c|c|}
\hline Name & Type & Antigen & $\begin{array}{l}\text { Immunized } \\
\text { Species }\end{array}$ & Reference \\
\hline MSC 1 & Polyclonal & $\begin{array}{l}\text { tail peptide of } \\
\text { MPR46 }\end{array}$ & Rabbit & $\begin{array}{c}\text { (Klumperman et al., } \\
1993)\end{array}$ \\
\hline KII5 & Polyclonal & human MPR46 & Rabbit & \\
\hline$\gamma$-adaptin & Monoclonal & $\begin{array}{l}\text { Mouse } \gamma \text {-adaptin } \\
\text { hinge region }\end{array}$ & Mouse & $\begin{array}{l}\text { Transduction } \\
\text { laboratories }\end{array}$ \\
\hline$\alpha$-adaptin & Monoclonal & $\begin{array}{c}\text { Mouse } \alpha_{A} \text {-adaptin } \\
N \text { - terminus }\end{array}$ & Mouse & $\begin{array}{l}\text { Transduction } \\
\text { Laboratories }\end{array}$ \\
\hline 15 & Polyclonal & Rat MPR-300 & Rabbit & $\begin{array}{c}\text { (Claussen et al., } \\
\text { 1995) }\end{array}$ \\
\hline TIP47 & Polyclonal & $\begin{array}{l}\text { Synthetic peptide } \\
\text { of TIP47 amino } \\
\text { terminus aa } 1-16\end{array}$ & Guinea pig & Progen Biotechnik \\
\hline TIP47 & $\begin{array}{l}\text { Affinity } \\
\text { purified } \\
\text { polyclonal }\end{array}$ & His-tagged TIP47 & Rabbit & $\begin{array}{c}\text { (Barbero et al., 2001; } \\
\text { Diaz and Pfeffer, } \\
\text { 1998) }\end{array}$ \\
\hline PDI & Monoclonal & $\begin{array}{l}\text { Synthetic peptide } \\
\text { residues 499-509 }\end{array}$ & Mouse & StressGen \\
\hline
\end{tabular}




\begin{tabular}{|c|c|c|c|c||}
\hline GM130 & Monoclonal & $\begin{array}{c}\text { C-terminus of rat } \\
\text { protein } \\
\text { residues 869-982 }\end{array}$ & Mouse & $\begin{array}{c}\text { Transduction } \\
\text { Laboratories }\end{array}$ \\
\hline HA & $\begin{array}{c}\text { Monoclonal, } \\
\text { purified }\end{array}$ & Mono HA-tag & Mouse & Covance \\
\hline His & Monoclonal & RGS-HHHH & Mouse & Mouse \\
\hline Lamp1 & Monoclonal & Human lamp1 & Hybridoma Bank \\
\hline LDH & Polyclonal & Rabbit & $\begin{array}{c}\text { Prof.Dr. Söling, MPI } \\
\text { for Biophysical } \\
\text { Chemistry, Göttingen }\end{array}$ \\
\hline Rab9 & Monoclonal & $\begin{array}{c}\text { Recombinant } \\
\text { canine rab9 }\end{array}$ & Mouse & $\begin{array}{c}\text { Affinity Bioreagents, } \\
\text { INC }\end{array}$ \\
\hline \hline
\end{tabular}

Table 3.1. List of antibodies used in this study.

\subsubsection{Secondary antibodies}

Goat anti-mouse and goat anti-rabbit antibodies conjugated with Alexa633 were from Molecular Probes, other secondary antibodies listed below were purchased from Dianova, Hamburg.

Donkey anti-goat Texas Red conjugate

Goat anti-guinea pig Flourescein isothiocyanate conjugate

Goat anti-guinea pig Horseradish peroxidase conjugate

Goat anti-mouse Horseradish peroxidase conjugate

Goat anti-rabbit Horseradish peroxidase conjugate

Goat anti-mouse Cy2 conjugate

Goat anti-rabbit Cy2 conjugate

Goat anti-mouse Cy3 conjugate

Goat anti-rabbit Cy3 conjugate 


\subsubsection{Bacterial strains and mammalian cell lines}

\section{Escherichia coli:}

\begin{tabular}{|c|c|c|}
\hline Strain & Genotype & Firm \\
\hline $\mathrm{DH} 5 \alpha$ & $\begin{array}{l}\text { supE44, thi-1, recA1, relA1, hsdR17(rK-mK+), } \\
\text { thi-1, } \Delta \text { lacU169 (Ф80 lacZ } \Delta M 15) \text {, endA1, gyrA } \\
\left(\mathrm{Nal}^{\prime}\right)\end{array}$ & $\begin{array}{l}\text { Gibco BRL, } \\
\text { Eggenstein }\end{array}$ \\
\hline XL1-Blue & $\begin{array}{l}\text { recA1, endA1, gyrA96, thi-1, hsdR17, supE44, } \\
\left.\text { relA1, lac [F', proAB, lacl }{ }^{9} Z \Delta M 15, T n 10\left(\text { Tet }^{r}\right)\right]^{c}\end{array}$ & $\begin{array}{l}\text { Stratagene, } \\
\text { Heidelberg }\end{array}$ \\
\hline $\begin{array}{l}\text { BL21- } \\
\text { (D3)-RIL }\end{array}$ & $\begin{array}{l}\text { E. coli B F ompT hsdS }\left(\mathrm{r}_{\mathrm{B}}^{-} \mathrm{m}_{\mathrm{B}}^{-}\right) \mathrm{dcm}^{+} \mathrm{Tet}^{\mathrm{r}} \text { gal } \\
\left.\lambda(\mathrm{DE} 3) \text { endA Hte [argU ileY leuW Cam}{ }^{r}\right]\end{array}$ & $\begin{array}{l}\text { Stratagene, } \\
\text { Heidelberg }\end{array}$ \\
\hline
\end{tabular}

\section{Mammalian cell lines}

Cos 7: green monkey kidney cells

HeLa: human cervical carcinoma cells

HeLa cells stably transfected with TIP47 anti-sense construct (this work)

\subsubsection{Media and Solutions for Cell culture}

PBS (Sterile for cell culture)

$150 \mathrm{mM} \mathrm{NaCl}$

$120 \mathrm{mM} \mathrm{KCl}$

$10 \mathrm{mM} \mathrm{Na}_{2} \mathrm{HPO}_{4} / \mathrm{KH}_{2} \mathrm{PO}_{4}, \mathrm{pH} 7.4$

Dulbecco's Modified Eagles Medium

(DMEM)

Gibco-Invitrogen

Fetal Calf Serum (FCS)

PAA laboratories $\mathrm{GmbH}$, Austria

Trypsin-EDTA solution (1x)

$0.5 \mathrm{~g} / \mathrm{L}$ Trypsin

$0.2 \mathrm{~g} / \mathrm{L}$ EDTA

in Puck's Salt Solution A without $\mathrm{Ca}^{2+}$

and $\mathrm{Mg}^{2+}$

Gibco

L-Glutamine $(100 \mathrm{x}) \quad$ Gibco

(200 mM)

Phosphates, pyruvate-free DMEM PAN Biotech GmbH

Sulfate-free DMEM powder

PAN Biotech $\mathrm{GmbH}$

\subsubsection{Stock solutions and buffers}

$10 \times$ PBS

$100 \mathrm{mM}$ sodium phosphate buffer $\mathrm{pH} 7.4$ 
$9 \%$ sodium chloride

Dissolved in $800 \mathrm{ml}$ water, volume was made up to $1000 \mathrm{ml}$ and autoclaved. Stored at room temperature.

$1 \times$ TBS

$10 \mathrm{mM}$ Tris/ $\mathrm{HCl}$ pH 7,4

$150 \mathrm{mM}$ Sodium chloride

$1 \times$ TAE

0.04 M Tris-acetate

$1 \mathrm{mM}$ EDTA (pH 8.0)

$\underline{50 \times \mathrm{TAE}}$

$242 \mathrm{~g}$ Tris base

$57.1 \mathrm{~g}$ glacial acetic acid

$100 \mathrm{ml}$ of $0.5 \mathrm{M}$ EDTA ( $\mathrm{pH} 8.0$ )

Dissolved in water and the final volume was made upto one litre.

$\underline{\text { TE Buffer }}$

$10 \mathrm{mM}$ Tris/ $\mathrm{HCl} \mathrm{pH} 7,5$

$1 \mathrm{mM}$ EDTA

\subsubsection{Computing facilities used}

Hardware

Notebook Intel® Celeron ${ }^{\mathrm{TM}} \mathrm{CPU}$

Gericom

Power Macintosh 7600/132

Apple Computers Inc

Laser Jet $4050 \mathrm{~N}$

Hewlett Packard, Palo Alto (USA)

Scanner ScanJet 4C/T

Hewlett Packard, Palo Alto (USA)

\section{Software}

Adobe Acrobat 5.0

Adobe System Inc

Adobe Illustrator 10

Adobe System Inc

Adobe Photoshop 6.0

Adobe System Inc

Aida image analyzer

raytest

EndNote 4.0

ISI ResearchSoft 
Image gauge

Image reader

Leica Confocal Software

Microsoft Office 2000 Professional

TILLvisION v4.0

Windows XP

Image Reader LAS-1000

(Pro Vers. 2.1)
Fuji

Fuji

Leica Microsystems Heidelberg $\mathrm{GmbH}$

Microsoft Corporation

TILL Photonics GmbH, Germany

Microsoft Corporation

raytest, Straubenhardt

\subsection{Methods}

\subsubsection{METHODS OF CELL BIOLOGY}

\subsubsection{Cell Culture}

All cells were grown in a humidified incubator with $5 \% \mathrm{CO}_{2}$ at $37^{\circ} \mathrm{C}$. All media used for cell-culture were pre-warmed to $37^{\circ} \mathrm{C}$ in a water bath.

For culturing HeLa and Cos cells, Dulbecco's modified essential medium (DMEM) with $10 \%$ fetal calf serum (FCS), $2 \mathrm{mM}$ L-glutamine and $100 \mathrm{U} / \mathrm{ml}$ Penicillin/Streptomycin was used.

For cells stably transfected with plasmids encoding Neomycin resistance, the medium was supplemented with $800 \mu \mathrm{g} / \mathrm{ml}$ Neomycin.

\subsubsection{Passaging of cells by trypsinization}

Cells grown to confluence were routinely passaged by trypsinization. The medium from confluent cells was aspirated and the cells were washed once with PBS, followed by incubation with trypsin-EDTA solution on a $37^{\circ} \mathrm{C}$ metal platform for about $5 \mathrm{~min}$. Detachment of the cells was examined under the microscope. Trypsin was inactivated by adding fresh medium into the flask and the cells were resuspended in this medium. About $1 / 3$ to $1 / 5$ of the volume was left in the flask, while the remaining cells were either used for experimental purposes or discarded. An appropriate 
volume of the medium was added to the flask and the cells were placed back into the incubator.

\subsubsection{Freezing cells for stock maintenance}

Freezing medium: Cell culture medium containing $10 \%$ DMSO

The trypsinized cells were collected in a centrifuge tube and were pelleted at $500 \mathrm{~g}$ for $5 \mathrm{~min}$. The supernatant was discarded, the cell pellet was resuspended in an appropriate volume of freezing medium. $1 \mathrm{ml}$ aliquots of the cell suspension were pipetted into freezing vials and stored at $-80^{\circ} \mathrm{C}$ overnight, before the frozen vials were transferred to liquid nitrogen. Usually from one confluent $35 \mathrm{~cm}^{2}$ flask, four vials were prepared.

\subsubsection{Thawing cells from liquid nitrogen}

Vials containing frozen cells were taken out of liquid nitrogen, the cap of the vial was loosened in the hood to release the the evaporating $\mathrm{N}_{2}$, tightened again and thawed in a beaker containing $70 \%$ prewarmed ethanol to $37^{\circ} \mathrm{C}$ in the waterbath. Subsequently, the cells were pipetted into a centrifuge tube containing chilled cell culture medium, and centrifuged for $5 \mathrm{~min}$ at $500 \mathrm{~g}$ to remove the DMSO. The cell pellet was resuspended with prewarmed medium, transferred to a new culture flask and kept in the incubator. For transfected cells, on day after thawing, the medium was replaced with medium containing appropriate selection antibiotics.

\subsubsection{Transfection with Effectene transfection reagent (Quiagen)}

Transfection with Effectine reagent was performed according to the manufacturer's prototcol in 4 well plates or in $3 \mathrm{~cm}$ dishes. The day before transfection, $7-25 \times 10^{4}$ cells were plated onto a $3 \mathrm{~cm}$ dish and grown to $10-20 \%$ confluency. $1 \mathrm{\mu g}$ of DNA was diluted in DNA-condensation buffer, Buffer EC, to a total volume of $100 \mu \mathrm{l} .3 .2 \mu \mathrm{l}$ of Enhancer was added and mixed by vortexing for a second and the resulting solution was incubated at room temperature for $5 \mathrm{~min}$. $10 \mu \mathrm{l}$ of Effectene transfection reagent was added to the DNA-Enhancer mixture and mixed by vortexing for 10 seconds. The samples were incubated for $10 \mathrm{~min}$ at room temperature to allow complex formation. During this incubation time, the old medium was aspirated from the cells and $1.6 \mathrm{ml}$ fresh medium was added. $600 \mu \mathrm{l}$ of the medium was added to the 
reaction tube containing the transfection complexes and mixed by pipetting up and down. Immediately after mixing, the transfection complex was added dropwise onto the cells and mixed gently by swirling the dish for uniform distribution of the complexes. The cells were incubated at $37^{\circ} \mathrm{C}$ and $5 \% \quad \mathrm{CO}_{2}$ to allow for gene expression. Next day, the medium was replaced with fresh medium and the cells were used for video-microscopy experiments or for selection of stably transfected clones. $24 \mathrm{~h}$ following transfection, selection for antibiotic resistant clones was started by adding medium containing an appropriate antibiotic. Antibiotic concentration was gradually increased by $100 \mu \mathrm{g}$ per day and stopped at $800 \mu \mathrm{g} / \mathrm{ml}$ of neomycin. Resistant clones were picked up and cultured as described (3.2.1.1). Stable clones were always maintained in the medium containing resistant antibiotic thereafter.

\subsubsection{MOLECULAR BIOLOGY METHODS}

\subsubsection{Cultivation of E.coli}

\section{Luria Bertani (LB) medium}

$10 \mathrm{~g}$ Bacto-Tryptone

$5 \mathrm{~g}$ Bacto-yeast extract

$5 \mathrm{~g} \mathrm{NaCl}$

Dissolved in $900 \mathrm{ml}$ distilled water, $\mathrm{pH}$ adjusted to 7.0 with $10 \mathrm{~N} \mathrm{NaOH}$, made up the volume to one liter, sterilized by autoclaving and stored at room temperature.

\section{$\underline{\text { LB-Ampicillin Agar Plates }}$}

$1.5 \%$ of Agar was added to the LB medium and autoclaved. After autoclaving, the medium was let to cool down to $55^{\circ} \mathrm{C}$ and ampicillin was added to a final concentration $100 \mu \mathrm{g} / \mathrm{ml}$. This medium was poured into $10 \mathrm{~cm}$ petriplates in the hood and left undisturbed for about $30 \mathrm{~min}$ to solidify. LB-Agar plates were stored in the cold room.

\subsubsection{Preparation of competent $E$. coli cells}

Buffers

TFB I $\quad 30 \mathrm{mM}$ Calcium acetate, $\mathrm{pH} 5.8$ 100 mM Rubidium chloride 10 mM Calcium chloride 
$50 \mathrm{mM}$ Manganese chloride

$15 \%$ Glycerol (w/v)

TFB II $\quad 10 \mathrm{mM}$ MOPS, $\mathrm{pH} 6.5$

$75 \mathrm{mM}$ Calcium chloride

$10 \mathrm{mM}$ Rubidium chloride

$15 \%$ (w/v) Glycerol

A 2-3 $\mathrm{ml}$ bacterial pre-culture was grown in LB medium at $37^{\circ} \mathrm{C}$ overnight. $1 \mathrm{ml}$ of the pre-culture was then inoculated into $99 \mathrm{ml}$ of LB medium and grown at $37^{\circ} \mathrm{C}$ to an OD600 of 0.4-0.6. Cells were pelleted at 6,000 RPM for $5 \mathrm{~min}$ at $4^{\circ} \mathrm{C}$, resuspended in 0.2 culture volume of TFB I and incubated on ice for $5 \mathrm{~min}$. Cells were centrifuged at 5,000 RPM for $5 \mathrm{~min}$ at $4^{\circ} \mathrm{C}$, the pellet was resuspended in 0.04 culture volume of TFB II and incubated on ice for $30 \mathrm{~min}$. Aliquots of $50 \mu \mathrm{l}$ were frozen at $-80^{\circ} \mathrm{C}$.

\subsubsection{Transformation of E.coli competent cells}

The competent bacteria were thawed on ice. 100-200 ng of DNA was added to each $50 \mu \mathrm{l}$ aliquot of competent cells and incubated on ice for $15 \mathrm{~min}$. Cells were subjected to heat shock by incubating at $42^{\circ} \mathrm{C}$ for $2 \mathrm{~min}$ and incubated on ice for $2 \mathrm{~min}$. $1 \mathrm{ml}$ of LB medium was added to the cells followed by incubation at $37^{\circ} \mathrm{C}$ in the shaker for 40min. Cells were plated on LB-agar plates containing appropriate antibiotic.

\subsubsection{Preparation of electrocompetent cells}

$10 \%(\mathrm{v} / \mathrm{v})$ Glycerol : $1 \mathrm{ml}(1.26 \mathrm{~g})$ of glycerol in $10 \mathrm{ml}$ sterile water

A single E. coli colony was inoculated into $10 \mathrm{ml}$ of LB media and allowed to grow overnight under constant shaking at $37^{\circ} \mathrm{C}$ in an incubator. This pre-culture was inoculated into $1 \mathrm{~L}$ LB medium and allowed to grow to an OD600 of $0.4-0.6$ at $37^{\circ} \mathrm{C}$. Cells were pre-chilled on ice for $15 \mathrm{~min}$ and then pelleted at 5000 RPM for $15 \mathrm{~min}$ at $4^{\circ} \mathrm{C}$. Pellet was resuspended in $1 \mathrm{~L}$ of ice cold sterile water and centrifuged as described above. The washing with $500 \mathrm{ml}$ water was repeated one more time. The pellet was resuspended in $20 \mathrm{ml}$ of $10 \%$ glycerol and centrifuged 6000 RPM 15min at $4^{\circ} \mathrm{C}$. The bacteria were resuspended in $2 \mathrm{ml}$ of $10 \%$ glycerol and aliquots of 40,80 and $160 \mu \mathrm{l}$ were frozen on dry ice and stored at $-80^{\circ} \mathrm{C}$. 


\title{
3.2.2.5 Transformation of the electrocompetent cells
}

SOC medium

\author{
$0.5 \%$ Yeast extract \\ $2 \%$ Bacto-tryptone \\ $10 \mathrm{mM}$ Sodium chloride \\ 2,5 mM Potassium chloride \\ $10 \mathrm{mM}$ Magnesium sulfate \\ $10 \mathrm{mM}$ Magnesium chloride \\ $20 \mathrm{mM}$ Glucose
}

For each electroporation $40 \mu \mathrm{l}$ of the electrocompetent cells were used. $0.5 \mu \mathrm{g}$ of DNA was added to the cells and the contents were transferred into a pre-chilled electroporation cuvette. The cuvette was placed in the electroporater and pulse was applied. SOC medium was added to the cells and they were allowed to recover in sterile tubes for $30 \mathrm{~min}$ at $37^{\circ} \mathrm{C}$ in a shaker incubator. Cells were plated on LB plates containing appropriate antibiotic.

\subsubsection{Glycerol stocks of bacterial strains}

Bacterial cultures were grown overnight at $37^{\circ} \mathrm{C}$ in a shaker-incubator. $0.25 \mathrm{ml}$ of sterile $80 \%$ glycerol was taken in $1.5 \mathrm{ml}$ tube to which $750 \mu \mathrm{l}$ of the overnight culture was added. The contents were gently mixed, shock frozen on dry ice and stored at $80^{\circ} \mathrm{C}$.

\subsubsection{Mini preparation of plasmid DNA}

Plasmid DNA was isolated from E.coli cultures using the kit provided by Qiagen. Isolation was done as follows according to the instructions of the manufacturer.

$\begin{array}{ll}\text { Buffer P1 (Resuspention buffer) } & 50 \mathrm{mM} \text { Tris/ HCl pH 8,0 } \\ & 10 \mathrm{mM} \text { EDTA } \\ & 100 \mu \mathrm{g} / \mathrm{ml} \text { RNase A } \\ & 0.2 \mathrm{M} \mathrm{NaOH} \\ \text { Buffer P2 (Lysis buffer) } & 1 \% \mathrm{SDS} \\ & 3 \mathrm{M} \text { Potassium acetate pH } 5.5\end{array}$

A single $E$. coli colony was inoculated into $2 \mathrm{ml}$ of LB medium containing $100 \mu \mathrm{g} / \mathrm{ml}$ of antibiotic and grown overnight at $37^{\circ} \mathrm{C}$ in a shaker incubator. Cells were pelleted in a 
table-top centrifuge at 13,000 RPM for $1 \mathrm{~min}$. Cell pellet was resuspended in $250 \mu \mathrm{l}$ of buffer P1 and $250 \mu \mathrm{l}$ of buffer P2 was added, mixed by gently inverting the tube 46 times. To this, $350 \mu$ of buffer P3 was added and gently mixed and centrifuged for $10 \mathrm{~min}$ at 13,000 RPM in a table-top eppendorf centrifuge. The supernatant was applied onto a QIAprep spin column and centrifuged for $1 \mathrm{~min}$ at 13,000 RPM. Flow through was discarded, the column was washed with $0.75 \mathrm{ml}$ of buffer PE and centrifuged again for $1 \mathrm{~min}$. Flow through was discarded and the column was centrifuged for an additional $1 \mathrm{~min}$ to remove any residual wash buffer. The column was placed in a clean eppendorf tube and $50 \mu \mathrm{l}$ of double distilled water was added directly to the centre of the column. The column was let to stand for 1 min and DNA was eluted by centrifuging at 13,000 RPM for $1 \mathrm{~min}$.

\subsubsection{Midi preparation of plasmid DNA}

Buffers P1, P2 and P3 are identical to buffers used for Mini preparations of DNA.

Buffer QBT (Equilibration buffer) $\quad 750 \mathrm{mM} \mathrm{NaCl}$

$50 \mathrm{mM}$ MOPS, pH 7.0

$15 \%$ isopropanol $(\mathrm{v} / \mathrm{v})$

$0.15 \%$ Triton $\mathrm{X}-100(\mathrm{v} / \mathrm{v})$

Buffer QC (Wash buffer) $1 \mathrm{M} \mathrm{NaCl}$

50mM MOPS, pH 7.0

$15 \%$ isopropanol $(\mathrm{v} / \mathrm{v})$

Buffer QF (Elution buffer) $\quad 1.25 \mathrm{M} \mathrm{NaCl}$

50mM Tris- $\mathrm{HCl} \mathrm{pH} 8.5$

$15 \%$ isopropanol $(\mathrm{v} / \mathrm{v})$

Buffer TE 10mM Tris- $\mathrm{HCl}, \mathrm{pH} 8.0$

$1 \mathrm{mM}$ EDTA

For preparation of up to 200 $\mathrm{\mu g}$ of plasmid DNA HiSpeed Plasmid Midi Kit (Qiagen) was used. A single $E$. coli colony was inoculated into $2 \mathrm{ml}$ of LB medium containing $100 \mu \mathrm{g} / \mathrm{ml}$ of antibiotic and grown overnight at $37^{\circ} \mathrm{C}$ in a shaker incubator. Then the starter culture was inoculated in $150 \mathrm{ml}$ medium and grown overnight with vigorous shaking. The bacteria were harvested by centrifugation at 6,000 RPM for 15min at 
$4^{\circ} \mathrm{C}$. The pellet was resuspended in $6 \mathrm{ml}$ buffer $\mathrm{P} 1$, gently mixed with $6 \mathrm{ml}$ buffer $\mathrm{P} 2$ by inverting 5 times and incubated for $5 \mathrm{~min}$ at RT. The lysate was mixed by inverting 5 times with $6 \mathrm{ml}$ of buffer P3, poured into the barrel of the QIAfilter cartridge and incubated at RT for $10 \mathrm{~min}$. Meanwhile, HiSpeed Midi Tip was equilibrated by applying $4 \mathrm{ml}$ buffer QBT. Then the HiSpeed Midi Tip was emptied by gravity flow, the cell lysate was filtered into the Tip and entered the resin by gravity flow. The HiSpeed Midi Tip, containing DNA, was washed with $20 \mathrm{ml}$ buffer QC. Afterwards the DNA was eluted with $5 \mathrm{ml}$ buffer QF and precipitated by adding $3.5 \mathrm{ml}$ isopropanol, mixing and incubating for $5 \mathrm{~min}$ at RT. Then the eluate/isopropanol mixture was filtered through the QIAprecipitator. The QIAprecipitator was dried by pressing air through the membrane and the DNA was eluted in $0.5 \mathrm{ml}$ water.

\subsubsection{Determining the concentration of DNA}

DNA concentration was determined using a spectrophotometer at $260 \mathrm{~nm} .1 \mu \mathrm{l}$ DNA was diluted in $100 \mu \mathrm{l}$ water and the absorbance was measured at $260 \mathrm{~nm}$.

Absorbance or optical density (OD) of 1 at $260 \mathrm{~nm}$ corresponds to $\sim 50 \mu \mathrm{g} / \mathrm{ml}$ of double stranded DNA or $\sim 40 \mu \mathrm{g} / \mathrm{ml}$ of single stranded DNA and RNA or $\sim 20 \mu \mathrm{g} / \mathrm{ml}$ of oligonucleotides. The ratio between the readings at $260 \mathrm{~nm}$ and $280 \mathrm{~nm}\left(\mathrm{OD}_{260} /\right.$ $\mathrm{OD}_{280}$ ) provides an estimate of the purity of the nucleic acid. Pure preparations of DNA and RNA have $\mathrm{OD}_{260} / \mathrm{OD}_{280}$ values of 1.8 and 2.0 , respectively. Any contamination with proteins or phenol would decrease the values mentioned above.

\subsubsection{Amplification of DNA by polymerase chain reaction (PCR)}

10xPCR buffer $100 \mathrm{mM} \mathrm{KCl}$ 100mM Tris- $\mathrm{HCl}, \mathrm{pH} 9.0$

The polymerase chain reaction (PCR) with specific primers was used for amplification of plasmid DNA fragments. The reaction mixture contained

Template DNA

10xPCR buffer

dNTP mixture $(1.25 \mathrm{mM})$

5 '-primer $(10 \mathrm{pmol} / \mu \mathrm{ll})$

3'-primer (10pmol/ $/ \mathrm{ll})$

Taq polymerase $(\mathrm{U} / \mathrm{\mu l})$ 100-500ng

$10 \mu \mathrm{l}$

$2 \mu \mathrm{l}$

$1 \mu \mathrm{l}$

$1 \mu \mathrm{l}$

$0.5 \mu \mathrm{l}$ 
Total volume was adjusted to $100 \mu \mathrm{l}$ with $\mathrm{ddH}_{2} \mathrm{O}$.

Usually 25-35 three step cycles were used:

1) $30 \mathrm{~s}$ at $95^{\circ} \mathrm{C}$ for template denaturation

2) $90 \mathrm{~s}$ at $48-58^{\circ} \mathrm{C}$ annealing of primers to template

3) $1 \mathrm{~min} / \mathrm{kb}$ of the product at $72^{\circ} \mathrm{C}$ (for Taq polymerase) elongation of the PCR product

\subsubsection{Purification of PCR products}

To purify PCR products ranging from $100 \mathrm{bp}$ to $10 \mathrm{~kb}$ from primers, nucleotides, polymerases, and salts, QIAquick PCR purification kit was used. 1 volume of the PCR reaction was mixed with 5 volumes of buffer PB, applied to the QIAquick column and centrifuged 30 s at 13,000 RPM in a microcentrifuge. The flow-through was discarded, the column was washed with $750 \mu l$ buffer PE and centrifuged for one additional time $1 \mathrm{~min}$ at maximum speed to remove residual washing buffer. DNA was eluted with $50 \mu \mathrm{ldd} \mathrm{H}_{2} \mathrm{O}$.

\subsubsection{Restriction endonuclease digestion of DNA}

The activity of restriction enzymes is measured in terms of 'Units' (U).

One unit of restriction enzyme is the amount of enzyme required to completely digest $1 \mu \mathrm{g}$ substrate DNA in $1 \mathrm{~h}$.

Plasmid DNA $\quad 0.5-1 \mu \mathrm{g}$

$10 \times$ buffer $\quad 1.5 \mu \mathrm{l}$

Restriction enzyme $\quad 1-2 \mathrm{U}$

BSA $1 \mu \mathrm{g} / \mu \mathrm{l}$ (added where essential)

Water was added to a final volume of $15 \mu$. Reaction mix was incubated at $37^{\circ} \mathrm{C}^{*}$ for 2 hours.

*Incubation temperatures were set as recommended by the manufacturer (New England Bio Labs Beverly U.S.A.) which varies from enzyme to enzyme. Reaction mix was analyzed on an agarose gel ( 0.8 to $2 \%$ depending on the size of the DNA of interest)

The buffers and enzymes used were all from New England Biolabs. 
The composition of the buffers is as follows:

NEB 1: $10 \mathrm{mM}$ Bis Tris Propane-HCl (pH 7.0), $10 \mathrm{mM} \mathrm{MgCl}_{2}, 1 \mathrm{mM}$ DTT

NEB 2: $10 \mathrm{mM}$ Tris- $\mathrm{HCl}$ (pH 7.9), $10 \mathrm{mM} \mathrm{MgCl}_{2}, 50 \mathrm{mM} \mathrm{NaCl}, 1 \mathrm{mM}$ DTT

NEB 3: 50 mM Tris- $\mathrm{HCl}(\mathrm{pH}$ 7.9), $10 \mathrm{mM} \mathrm{MgCl} 2,100 \mathrm{mM} \mathrm{NaCl}, 1 \mathrm{mM}$ DTT

NEB 4: $20 \mathrm{mM}$ Tris-acetate (pH 7.9), $10 \mathrm{mM}$ Magnesium acetate, $1 \mathrm{mM}$ DTT, $50 \mathrm{mM}$ Potassium acetate

\subsubsection{Agarose gel electrophoresis of DNA}

The size and purity of DNA was analyzed by agarose gel electrophoresis. Concentration of agarose used for analysis is inversely proportional to the size of the DNA of interest, that is, the larger the DNA the lower the concentration of agarose.

\begin{tabular}{|c|c|}
\hline Agarose concentration (\%) & DNA size $(\mathrm{kb})$ \\
\hline 0.7 & $20-1$ \\
\hline 0.9 & $7-0.5$ \\
\hline 1.2 & $6-0.4$ \\
\hline 1.5 & $4-0.2$ \\
\hline 2.0 & $3-0.1$ \\
\hline
\end{tabular}

\section{Gel loading buffer (10x)}

$0.25 \%(w / v)$ Bromophenol blue

$40 \%$ Saccharose in $1 \times$ TAE

Agarose was weighed and dissolved in $1 \mathrm{x}$ TAE by boiling in a microwave oven. The agarose solution was allowed to cool down to $60^{\circ} \mathrm{C}$ or lower before ethidium bromide was added to a final concentration of $0.5 \mu \mathrm{g} / \mathrm{ml}$. The solution was poured into the agarose gel cassette and allowed to polymerize completely. The sample DNA was mixed with gel loading buffer and loaded into the lane. The gel electrophoresis was carried out at $100 \mathrm{~V}$. Ethidium bromide is a fluorescent dye which contains a planar group that intercalates between the stacked bases of the DNA. The fixed position of 
this group and its close proximity to the bases cause dye bound to DNA to display an increased fluorescent yield compared to that of the dye in free solution. Ultraviolet radiation at $254 \mathrm{~nm}$ is absorbed by the DNA and transmitted to the dye; radiation at $302 \mathrm{~nm}$ and $366 \mathrm{~nm}$ is absorbed by the bound dye itself. In both cases, the energy is re-emitted at $590 \mathrm{~nm}$ in the red orange region of the visible spectrum. Hence DNA can be visualized under a UV transilluminator. The gel was photographed using a gel documentation system.

\title{
3.2.2.14 Extraction of DNA fragments from agarose gels
}

DNA fragments separated by agarose gel electrophoresis were extracted and purified by means of QIAquick gel extraction kit. DNA fragment of interest was excised from the agarose gel. Resulting gel slice was weighted, submerged into 3 volumes of buffer $Q G$ to 1 volume of gel and incubated at $50^{\circ} \mathrm{C}$ for $10 \mathrm{~min}$ (or until the gel slice has completely dissolved). The DNA solution was applied to the QIAquick column and centrifuged for $1 \mathrm{~min}$. The flow-through was discarded, the DNA bound to the column was processed further as described in 3.2.2.11.

\subsubsection{Ligation of DNA fragments}

1x T4 DNA ligase reaction buffer

\author{
50mM Tris- $\mathrm{HCl}, \mathrm{pH} 7.5$ \\ $10 \mathrm{mM} \mathrm{MgCl} 2$ \\ $10 \mathrm{mM}$ dithiothreitol \\ $1 \mathrm{mM}$ ATP \\ $25 \mu \mathrm{g} / \mathrm{ml} \mathrm{BSA}$
}

The activity of ligases is measured in terms of 'Units' (U). One NEB unit is defined as the amount of enzyme required to give $50 \%$ ligation of Hind III fragments of $\lambda$ DNA (5'-termini concentration of $0.12 \mu \mathrm{M})$ in $20 \mu \mathrm{l}$ of T4 DNA ligase reaction buffer in 30 $\min$ at $16^{\circ} \mathrm{C}$.

T4 DNA ligase $(400 \mathrm{U} / \mu \mathrm{l}) \quad 1.5 \mu \mathrm{l}$

Vector DNA fragment 10-15ng

Insert DNA fragment 100-200ng

$10 \times$ buffer $2 \mu \mathrm{l}$ 
Water was added to a final volume of $20 \mu$ l. Ligation mixture was incubated at RT for 2 hours or at $16^{\circ} \mathrm{C}$ overnight.

\subsubsection{Cloning of full length and truncated TIP47 cDNA}

Full length Homo sapiens TIP47 cDNA was cloned by RT-PCR using total RNA isolated by the RNAeasy method (Quiagen) from HeLa cells and primers matching to the coding region of human TIP47 mRNA. 5'-primers included Xhol cloning site and HA-, RGS-His- or no tag; 3'-primers introduced HA-, RGS-His- or no tag as well as BamHI restriction site after the stop codon (Table 3.2). These primers were necessary to generate proteins, containing the tag at the $\mathrm{C}$ - or $\mathrm{N}$-terminus:

HA-TIP47 TIP47-HA

His-TIP47 TIP47-His

Amplified DNAs were cloned into the pMPSV-EH vector with Xhol/BamHI cloning sites and sequenced (3.2.2.18).

TIP47 protein was conventionally divided into 3 parts (ссылка на резельтаты):

The PAT domain

homologous to LD targeting domain

of ADRP (McManaman et al., 2003)

coiled-coil rich region

So 5 constructs for mammalian expression of truncated proteins

HA-TIP47 1-116

HA-TIP47 1-239

HA-TIP47 117-239

TIP47-HA 117-434

TIP47-HA 240-434

were generated as described above, sequenced (3.2.2.18) and expressed in HeLa cells (3.2.1.5).

1-116 aa residues

117-239 aа

240-433 aa 
Table 3.2. Primers used for cloning of full length and truncated TIP47. Xhol and BamHI cloning sites (5'- and 3'-, respectively) are underlined, start and stop codons are shown in lowercase letters. HAand His-tags are italicized.

\begin{tabular}{|c|c|c|}
\hline Primer specificity & Orientation & Sequence $5^{\prime} \rightarrow 3^{\prime}$ \\
\hline $\begin{array}{l}\text { Including Xhol site } \\
\text { and start codon }\end{array}$ & 5 & $\begin{array}{l}\text { TCTGCCCGCTCGAGCatgTCTGCCGACGG } \\
\text { GG }\end{array}$ \\
\hline $\begin{array}{l}\text { Including BamHI } \\
\text { site and stop } \\
\text { codon }\end{array}$ & $3^{\prime}$ & TCCGCGGATCCCctaCTTCTTCTCCTC \\
\hline $\begin{array}{l}\text { Introducing RGS- } \\
\text { His-tag at N- } \\
\text { terminus of full } \\
\text { length TIP47 }\end{array}$ & 5 & $\begin{array}{l}\text { TCTGCCCGCTCGAGCatgAGAGGATCGCA } \\
\text { TCACCATCACCATCACATGTCTGCCGAC } \\
\text { GGGGC }\end{array}$ \\
\hline $\begin{array}{l}\text { Introducing HA-tag } \\
\text { at N-terminus of } \\
\text { full length TIP47 }\end{array}$ & $5^{\prime}$ & $\begin{array}{l}\text { TCTGCCCGCTCGAGCatgTATCCGTATGA } \\
\text { TGTGCCTGACTACGCAatgTCTGCCGACG } \\
\text { GGGC }\end{array}$ \\
\hline $\begin{array}{l}\text { Introducing RGS- } \\
\text { His-tag at C- } \\
\text { terminus of full } \\
\text { length TIP47 }\end{array}$ & $3^{\prime}$ & $\begin{array}{l}\text { TCCGCGGATCCCCtaGTGATGGTGATGGT } \\
\text { GATGCGATCCTCTCTTCTTCTCCTCCGGG } \\
\text { G }\end{array}$ \\
\hline $\begin{array}{l}\text { Introducing HA-tag } \\
\text { at C-terminus of } \\
\text { full length TIP47 }\end{array}$ & $3^{\prime}$ & $\begin{array}{l}\text { TCCGCGGATCCCCtaTGCGTAGTCAGGCA } \\
\text { CATCATACGGATACTTCTTCTCCTCCGGG } \\
\text { G }\end{array}$ \\
\hline $\begin{array}{l}\text { Introducing stop } \\
\text { codon after K116 }\end{array}$ & 3 & $\begin{array}{l}\text { TCCGCGGATCCCctaCTTCTCCGTGGGCT } \\
\text { GCTG }\end{array}$ \\
\hline $\begin{array}{l}\text { Introducing start } \\
\text { codon and HA-tag } \\
\text { before V117 }\end{array}$ & 5 & $\begin{array}{l}\text { TCTGCCCGCTCGAGCatgTATCCGTATGA } \\
\text { TGTGCCTGACTACGCAGTCCTGGCGGAC } \\
\text { ACCAAG }\end{array}$ \\
\hline $\begin{array}{l}\text { Introducing start } \\
\text { codon before V117 }\end{array}$ & $5^{\prime}$ & $\begin{array}{l}\text { TCTGCCCGCTCGAGCatgGTCCTGGCGGA } \\
\text { CACCAAG }\end{array}$ \\
\hline $\begin{array}{l}\text { Introducing stop } \\
\text { codon after L239 }\end{array}$ & 3' & $\begin{array}{l}\text { TCCGCGGATCCCCtaCAGACGTACGAAGT } \\
\text { AGCTC }\end{array}$ \\
\hline Introducing start & $5^{\prime}$ & TCTGCCCGCTCGAGCatgGGCTCCCTGTC \\
\hline
\end{tabular}




\begin{tabular}{|l|l|l|}
\hline $\begin{array}{l}\text { codon before } \\
\text { G240 }\end{array}$ & GGAGAG \\
\hline
\end{tabular}

\subsubsection{Cloning of TIP47 RNAi constructs and establishing of RNAi HeLa cell line}

GeneSilencer, a plasmid-based system for RNA interference (RNAi), was used to decrease TIP47 expression level in HeLa cells. The technique is based on ability of small interfering RNAs (siRNAs) to suppress gene expression. Introduction of double-stranded RNA (dsRNA), that are homologous in sequence to a gene, has proven to suppress gene expression without producing a non-specific cytotoxic response (Elbashir et al., 2001). Inserts cloned into GeneSilencer vector pSHH express RNAs under U6 promoter in the transfected mammalian cells. The RNAs are expressed as fold-back stem loop structure, which are processed into the siRNAs. The oligonucleotide inserts were designed as follows: target region of TIP47 mRNA in direct and reversed orientation were separated by short loop sequence (Brummelkamp et al., 2002). 4 nucleotide overhang for ligation with Sall/Xhol site and TTTTT as terminator of transcription were added at the 5'- or 3'- end of the resulting sequence, respectively. Overhang for ligation with Xbal site was added to the complementary sequence at 5'-end before AAAAA. The oligonucleotides (Table 3.3) were annealed $10 \mathrm{~min}$ at $95^{\circ} \mathrm{C}$ and ligated with linearized vector $\mathrm{pSHH}$. These plasmids were sequenced (see chapter 3.2.2.18) and transfected in HeLa cell line. Clones resistant for upto $800 \mu \mathrm{g} / \mathrm{ml}$ neomycin were picked and cultured for further experiments. Decrease in TIP47 expression level was tested by western-blot using commercial guinea pig or affinity purified rabbit anti-TIP47 antibodies (kind gift of Dr. S.R.Pfeffer).

Table 3.3. Olygonucleotides used for plasmid based RNAi. Underlined letters represent sticky ends for Xhol/Sall sites (5'-primer) and Xbal sites (3'- primer). Capital letters show target region of the gene in direct and reverse orientation. The stem loop is shown in lowercase letters.

\begin{tabular}{|l|l|l|}
\hline $\begin{array}{l}\text { Oligonucleotide } \\
\text { specificity }\end{array}$ & Orientation & Sequence $5^{\prime} \rightarrow 3^{\prime}$ \\
\hline RNAi of TIP47 1 & $5^{\prime}$ & $\begin{array}{l}\text { TCGAGGACACGGTGGCCACCCAAttcaaga } \\
\text { gaTTGGGTGGCCACCGTGTCCTTTTT }\end{array}$ \\
\hline
\end{tabular}




\begin{tabular}{|l|l|l|}
\hline RNAi of TIP47 1 & $3^{\prime}$ & $\begin{array}{l}\text { CTAGAAAAAGGACACGGTGGCCACCCAAt } \\
\text { ctcttgaaTTGGGTGGCCACCGTGTCC }\end{array}$ \\
\hline RNAi of TIP47 2 & $5^{\prime}$ & $\begin{array}{l}\text { TCGACCCGGGGCTCATTTCAAACttcaagag } \\
\text { aGTTTGAAATGAGCCCCGGGTTTTT }\end{array}$ \\
\hline RNAi of TIP47 2 & $3^{\prime}$ & $\begin{array}{l}\text { CTAGAAAAACCCGGGGCTCATTTCAAACt } \\
\text { ctcttgaaGTTTGAAATGAGCCCCGGG }\end{array}$ \\
\hline
\end{tabular}

\subsubsection{DNA sequencing}

The sequencing of DNA was based on the method of Sanger and coworkers (1977) with some modifications. ABI PRISM® Rhodamine Terminator Cycle sequencing Ready Reaction Kit (Perkin Elmer Applied Biosystems, Weiterstadt, Germany) was used for sequencing. The kit contains rhodamine derivatives of di-desoxynucleotides which differ in their absorption and emission spectra depending on the ddNTP. The rhodamine-labeled ddNTPs were incorporated into the synthesized DNA during elongation of DNA chains in a sequence PCR. The reaction mixture contained: Terminator Ready Reaction Mix $2 \mu l$

Plasmid DNA 200-300ng

Primer $1 \mathrm{pM}$ $\mathrm{ddH}_{2} \mathrm{O}$ was added to a final volume of $10 \mu \mathrm{l}$.

The primers used for the reaction are gene-specific or correspond to sequences present in vectors themselves (Table 3.4.).

Amplification cycle, as follows, was repeated 25 times.

$96^{\circ} \mathrm{C} \quad 10 \mathrm{~s}$

$50^{\circ} \mathrm{C} \quad 5 \mathrm{~s}$

$60^{\circ} \mathrm{C} \quad 4 \mathrm{~min}$

After the PCR, the DNA was precipitated with ethanol to remove free rhodaminelabeled ddNTPs. $2 \mu \mathrm{l}$ of sodium acetate $\mathrm{pH} 5.2$ were mixed with $50 \mu \mathrm{l} 96 \%$ ethanol. The PCR product was added to the solution, mixed, incubated for $10 \mathrm{~min}$ at room temperature and centrifuged 13,000 RPM for 20min. The pellets were briefly washed with $250 \mu \mathrm{l}$ of $70 \%$ ethanol and spin down for $10 \mathrm{~min}$ at $13,000 \mathrm{RPM}$. The pellets were dried for $5 \mathrm{~min}$ and resolved in $25 \mu \mathrm{l}$ of $\mathrm{dd}_{2} \mathrm{O}$. The samples were kept at $-20^{\circ} \mathrm{C}$ until 
sequencing in $\mathrm{ABI}$ PRISM ${ }^{\mathrm{TM}} 310$ or 3100 Genetic Analyser (Perkin Elmer Life Sciences Ltd, Cambrige, UK).

Table 3.4. Primers used for sequencing.

\begin{tabular}{|l|l|l|}
\hline Primer specificity & Orientation & Sequence 5 $^{\prime} \rightarrow 3^{\prime}$ \\
\hline pBEH and pMPSV & $5^{\prime}$ & GTTAACTGGTAAGTTTAG \\
\hline pBEH and pMPSV & $3^{\prime}$ & GCATTCTAGTTGTGGTT \\
\hline $\begin{array}{l}\text { pMPSV-EH before } \\
\text { Xhol cloning site }\end{array}$ & $5^{\prime}$ & CACAACCCCTCACTC \\
\hline TIP47 & $5^{\prime}$ & GAGAAGGGAGTGAGG \\
\hline TIP47 & $5^{\prime}$ & TGTCGGAGGCGGTG \\
\hline TIP47 & $5^{\prime}$ & GAACAGAGCTACTTCG \\
\hline TIP47 & $5^{\prime}$ & GGAACCAGAAGCAGC \\
\hline pSHH & $5^{\prime}$ & GTTTTAAAATTATGTTTTAAAATGG \\
\hline pSHH & $3^{\prime}$ & GGTAACCGACGTCAAGC \\
\hline
\end{tabular}




\subsubsection{BIOCHEMICAL METHODS}

\subsubsection{Analysis of proteins}

\subsection{Protein determination}

Bovine Serum Albumin (BSA) stock solution $1 \mathrm{mg} / \mathrm{ml}$

A standard curve was made using BSA in the concentration range of $2-16 \mu \mathrm{g}$. 3-10 $\mu \mathrm{l}$ of the sample was used for the protein estimation. When the sample contained no detergents, the volume was made up to $800 \mu \mathrm{l}$ with water. $200 \mu \mathrm{l}$ of the BIORAD reagent was added and the samples were incubated for $3-5 \mathrm{~min}$ at room temperature, $200 \mu \mathrm{l}$ from each tube was pipetted into ELISA strips and optical density was measured at $595 \mathrm{~nm}$ in the ELISA reader.

\subsection{Solubilisation of proteins}

$2 \times$ Laemmli buffer $125 \mathrm{mM}$ Tris-Cl, $\mathrm{pH} 6.8$

$$
\begin{aligned}
& 4 \% \text { SDS } \\
& 0.004 \% \text { Bromophenol blue } \\
& 20 \% \text { (w/v) Glycerol } \\
& 20 \mathrm{mM} \text { DTT }
\end{aligned}
$$

Proteins were resuspended in $1 \mathrm{x}$ Laemmli buffer. The resuspended proteins were boiled at $95^{\circ} \mathrm{C}$ for $5 \mathrm{~min}$, cooled on ice for $1 \mathrm{~min}$, centrifuged at 13,000 RPM for $2 \mathrm{~min}$ and resolved by SDS-PAGE.

\subsection{TCA precipitation of proteins}

Trichloroacetic acid (TCA) solution

$(100 \%)$

$2 \%$ Sodium acetate in ethanol
$100 \mathrm{~g}$ of TCA crystals was weighed and the volume was made up to $100 \mathrm{ml}$ with water $2 \mathrm{~g}$ of Sodium was dissolved in small amount of water and volume was made up to $100 \mathrm{ml}$ with ethanol

The proteins were precipitated by adding TCA to a final concentration of $10 \%$ and 
incubated on ice in the cold room overnight. The precipitated samples were centrifuged at $13,000 \mathrm{RPM}, 10 \mathrm{~min}, 4^{\circ} \mathrm{C}$. The supernatant was discarded and the pellets were washed $500 \mu \mathrm{l}$ of $2 \%$ sodium acetate solution in ethanol. The samples were again centrifuged as above, supernatant was discarded and the pellets were air-dried for 10-15 min at RT. The pellets were resuspended in 1x SDS-PAGE buffer, boiled and loaded on SDS-PAGE for further analysis.

\subsection{SDS Poly-Acrylamide Gel Electrophoresis (SDS-PAGE)}

\section{Buffers}

Running buffer (1x) $25 \mathrm{mM}$ Tris

192 mM Glycine

$0.1 \%$ SDS

Volume was made up with $\mathrm{ddH}_{2} \mathrm{O}$.

Separating gel buffer (4x) $1.5 \mathrm{M}$ Tris-Cl pH 8.8

$0.4 \%$ SDS

$0.4 \%$ APS

Volume was made up with $\mathrm{ddH}_{2} \mathrm{O}$.

Stacking gel buffer $(4 \mathrm{x}) \quad 0.5 \mathrm{M}$ Tris- $\mathrm{Cl} \mathrm{pH} 6.8$

$$
0.4 \%(w / v) \text { SDS }
$$

Acrylamide Solution $\quad 30 \%(\mathrm{w} / \mathrm{v})$ Acrylamide

$0.8 \%(w / v)$ Bisacrylamide

Ammonium per sulphate $10 \%(\mathrm{w} / \mathrm{v})$

Recrystallised ammonium peroxidisulphate (APS) was dissolved in double distilled water. This was prepared fresh every week.

Preparation of acrylamide solution for SDS-PAGE (for one minigel - $8 \mathrm{ml}$ ).

All volumes are in $\mathrm{ml}$ unless indicated otherwise. 


\begin{tabular}{|c|c|c|c|c|c|}
\hline Gel (\%) & 5 & 10 & 12.5 & 15 & $\begin{array}{c}3 \text { ml Stacking } \\
\text { Gel }(4.5 \%)\end{array}$ \\
\hline $\begin{array}{c}\text { Acrylamide/ } \\
\text { Bis-acrylamide }\end{array}$ & 1.33 & 2.67 & 3.3 & 4 & 0.45 \\
\hline $\begin{array}{c}4 \text { x resolving gel } \\
\text { buffer }\end{array}$ & 2 & 2 & 2 & 2 & $\begin{array}{c}4 \text { x stacking } \\
\text { gel buffer } \\
0.45\end{array}$ \\
\hline Water & 4.58 & 3.2 & 2.6 & 1.9 & 1.77 \\
\hline Temed & $8 \mu \mathrm{l}$ & $8 \mu \mathrm{l}$ & $8 \mu \mathrm{l}$ & $8 \mu \mathrm{l}$ & $4 \mu \mathrm{l}$ \\
\hline $10 \%$ APS & $80 \mu \mathrm{l}$ & $80 \mu \mathrm{l}$ & $80 \mu \mathrm{l}$ & $80 \mu \mathrm{l}$ & $40 \mu \mathrm{l}$ \\
\hline
\end{tabular}

SDS-PAGE is a biochemical technique wherein proteins are separated based on their molecular weight. The gel is discontinuous in that it has a stacking gel which is present on top of the resolving gel. SDS-PAGE can be used to separate proteins based on their native size (native SDS-PAGE) or their sub unit size (reducing SDSPAGE). Two clean glass plates were fixed together with a spacer of appropriate thickness $(0.75-1.5 \mathrm{mM})$ using holders or clips. The resolving gel was poured and isobutanol was layered on top of it. The gel was allowed to polymerise for about 10 min and isobutanol was thoroughly washed off using double distilled water. The stacking gel was poured on top of the resolving gel and a comb, of appropriate size and appropriate number of wells, was placed in the stacking gel and left undisturbed for about $10 \mathrm{~min}$. After polymerisation of the stacking gel, the combs were removed and the wells were cleaned with double distilled water to wash off any unpolymerised acrylamide. Boiled and denatured protein samples were loaded into the wells, the gel was placed in a tank with electrophoresis buffer and run at $100-125 \mathrm{~V}$ for $2 \mathrm{~h}$.

\subsection{Coomassie blue staining of Polyacrylamide gels}

Coomassie blue stain $\quad 0.5 \%(w / v)$ Coomassie blue

$50 \%(v / v)$ Methanol

$10 \%(v / v)$ Acetic acid

in double distilled water 
Destaining solution

$50 \%$ Methanol and $10 \%$ glacial acetic acid dissolved in double distilled water.

The gel was stained in Coomassie blue solution at room temperature for 1-2 hours and destained using the destaining solution overnight. After complete destaining the gel was washed with double distilled water for $15 \mathrm{~min}$ and then dried in a gel drier.

\subsection{Silver staining of polyacrylamide gels modified for MS}

\section{Buffers}

Fixer

$50 \% \mathrm{MeOH}$

$12 \%$ acetic acid

$0.05 \%$ formalin

in $\mathrm{ddH}_{2} \mathrm{O}$

Washing solution

$35 \%$ EthOH in dd $\mathrm{H}_{2} \mathrm{O}$

Sensitizing

$0.02 \% \mathrm{Na}_{2} \mathrm{~S}_{2} \mathrm{O}_{3}$ in $\mathrm{ddH}_{2} \mathrm{O}$

Silver nitrate

$0.2 \% \mathrm{AgNO}_{3}$

$0.076 \%$ formalin in $\mathrm{ddH}_{2} \mathrm{O}$

Developer

$6 \% \mathrm{Na}_{2} \mathrm{CO}_{3}$

$0.05 \%$ formalin

$0.0004 \% \mathrm{Na}_{2} \mathrm{~S}_{2} \mathrm{O}_{3}$ in $\mathrm{ddH}_{2} \mathrm{O}$

Stop solution

$50 \% \mathrm{MeOH}$

$12 \%$ acetic acid in $\mathrm{dd}_{2} \mathrm{O}$

The polyacrylamide gel was fixed for $2 \mathrm{~h}$ in fixer and washed 3 times with $35 \%$ EthOH for $20 \mathrm{~min}$ each. Then the gel was sensitized for $2 \mathrm{~min} 0.02 \% \quad \mathrm{Na}_{2} \mathrm{~S}_{2} \mathrm{O}_{3}$ and washed with $\mathrm{ddH}_{2} \mathrm{O} 3$ times for $5 \mathrm{~min}$. In order to stain the gel was submerged into silver nitrate for $20 \mathrm{~min}$, washed twice with $\mathrm{ddH}_{2} \mathrm{O}$ for $1 \mathrm{~min}$ and developed in sodium carbonate solution. Staining was stopped by $5 \mathrm{~min}$ incubation in the stop solution. The gel was stored in $10 \%$ EthOH at $4^{\circ} \mathrm{C}$ till mass spectrometry analysis. 


\subsection{Western blot analysis (wet method)}

Blot buffer

$25 \mathrm{mM}$ Tris

192 mM Glycine

$20 \%$ Methanol

were dissolved in water.

PBST

PBS containing $0.05 \%$ Tween-20

Blocking buffer PBST containing 5\% milk powder

\section{Primary antibodies}

anti-gamma adaptin

anti-alpha adaptin

anti-MPR46

anti-TIP47

\section{Secondary antibody}

HRP (Horseradish peroxidase)

conjugated Goat anti-guinea pig,

anti-rabbit and anti-mouse antibody

\section{Final concentration}

$1: 5,000$

$1: 5,000$

$1: 1,000$

$1: 1,000$

\section{Final concentration}

$1: 5,000$

Proteins separated by SDS-PAGE were transferred onto nitrocellulose membrane for western blot analysis as follows. 2 sheets of whatman paper and one piece on nitrocellulose membrane were cut according to the size of the gel. A pad and one sheet of paper was immersed in the blot buffer and placed in the chamber. The gel was also soaked in the blot buffer before placing on the paper. Then the membrane dipped in the blot buffer was placed on top of it and the air bubbles were removed. Another whatman paper sheets was dipped in the buffer and placed on top of the gel. A glass pipette was rolled on the set up to remove air bubbles and the second pad was placed on top of the stack. The proteins were transferred onto the membrane using current at $150 \mathrm{~V}$ for $90 \mathrm{~min}$. 
After the transfer, membrane was briefly washed with PBS, incubated in PBST containing $5 \%$ milk powder for 1 hour at room temperature or o/n at $4^{\circ} \mathrm{C}$ to block all non-specific interaction sites on the membrane. After blocking, the primary antibodies suitably diluted in PBST, were added onto the membrane and incubated 1-4h RT or overnight in the cold room on a rocker. The non-specifically bound antibody was washed off by incubating the blot on a rocker with PBST, changing the buffer once every $10 \mathrm{~min}$ for three times. The membrane was then incubated with the secondary antibody for 1 hour at room temperature and the membrane was washed 3 times, 10 min each, with PBST. The proteins were detected by chemiluminescence kit after an additional brief washing of the membrane with PBS.

\subsection{Detection technique. Chemiluminescent Substrate (PIERCE)}

The nitrocellulose membrane was incubated at room temperature for $1 \mathrm{~min}$ in enhancer and peroxide solutions mixed in a 1:1 ratio. The membrane was wrapped in a polythene sheet and signals were detected using a CCD camera.

\subsection{Stripping the antibody from the membrane}

Stripping buffer

100mM $\beta$-mercaptoethanol

$2 \%$ SDS

62.5mM Tris- $\mathrm{HCl} \mathrm{pH} 6.7$

The membrane was washed once with PBS for $5 \mathrm{~min}$ to wash off the chemiluminescent substrate, submerged in stripping buffer and incubated at $50-70^{\circ} \mathrm{C}$ for 30min with occasional agitation. Then the membrane was washed twice with PBST for 10min using large volume of the buffer, incubated in blocking buffer for $1 \mathrm{~h}$ followed by antibody of interest for next round of chemiluminescence.

\subsubsection{Metabolic labeling of cells with [35S]-Methionine/Cysteine}

Solutions

dFCS heat inactivated dialyzed fetal calf serum

Starvation medium DMEM without Methionine /Cysteine supplemented with 5 $\%$ dFCS and 2 mM Glutamine

35S-Methionine/Cysteine $10 \mu \mathrm{Ci} / \mu \mathrm{l}$ 
Labelling of starved cells with radioactive methionine and cysteine leads to the incorporation of these amino acids into newly synthesized proteins and helps in the detection of the protein of our interest. Cells were grown in normal DMEM medium till $70-80 \%$ confluency. Before labeling, the cells were washed with PBS 3 times and incubated with starvation medium for one hour. The cells were again washed once with PBS and incubated with labeling medium (starvation medium containing normally $100 \mu \mathrm{Ci} / \mathrm{ml}$ of $35 \mathrm{~S}-M e t h i o n i n e / C y s t e i n e)$ for required time periods. In pulsechase experiments, after pulse period, the cells were chased after washing twice with PBS and addition to the dishes normal culture medium DMEM with $10 \% \mathrm{FCS}$. The labeled cells were processed as per the protocol for the desired experiments.

\subsubsection{Phosphorylation of TIP47 in vivo}

Solutions

dFCS

heat inactivated dialyzed fetal calf serum

Starvation medium DMEM without phosphate and piruvate, suplemented with $5 \% \mathrm{dFCS}$ and $2 \mathrm{mM}$ Glutamine

[Y-32P]-ATP $\quad 10 \mathrm{mCi} / \mathrm{ml}$

To analyse the putative dependence of TIP47 phosphorylation level on nutritional status of the cell, HeLa cells were plated onto three $3 \mathrm{~cm}$ dishes and grown overnight. Then two of the dishes were continuously incubated under standard cell culture conditions, later washed five times with PBS and incubated for $1 \mathrm{~h}$ at $37^{\circ} \mathrm{C}$ in the starvation medium (samples 1,2) or, alternatively, preincubated in the same media containing $600 \mu \mathrm{M}$ oleic acid complexed to BSA (6:1) (sample 3). After the inculation starvation media were aspirated, cells were supplied with $600 \mu \mathrm{l}$ of the labeling medium containing $500 \mu \mathrm{Ci}$ of $[\mathrm{Y}-32 \mathrm{P}]-\mathrm{ATP}$ (sample 1,3 ) or with the same labeling medium supplemented with $600 \mu \mathrm{M}$ oleic acid (sample 2) and incubated for $6 \mathrm{~h}$. After the labeling cells were processed for immunoprecipitation as described below (3.2.3.4). 


\subsubsection{Immunoprecipitation of TIP47}

Solutions

Homo Buffer

$0.1 \times$ PBS

\section{PANSORBIN}

\section{PANSORBIN}

Protein-A sepharose

$\mathrm{NaCl}$ - Tween 20 solution
Working concentration

50mM Tris- $\mathrm{HCl}, \mathrm{pH} 7.4$

1\% Triton $\mathrm{X}-100$

0.5\% Sodium Deoxycholate

300mM Sodium chloride

with protease inhibitor cocktail

1:10 diluted PBS

Heat killed and inactivated Staphylococcus aureus cell suspension in PBS

Staphylococcus aureus cells

Washed five times with PBS

$1 \mathrm{M} \mathrm{NaCl}$

$0.5 \%$ Tween 20

"Cold" HeLa cells grown on 6cm dishes or labeled as described previously () were washed three times with PBS, scraped and pelletted by low speed centrifugation. The cells were resuspended in $1 \mathrm{ml}$ of Homo buffer and incubated for $10 \mathrm{~min}$ at $4^{\circ} \mathrm{C}$ rotating. The resulting cell lysates were centrifuged for $15 \mathrm{~min}$ with maximal speed in a table-top centrifuge. The supernatant was transferred into new tubes and mixed with $2 \mu \mathrm{l}$ of rabbit preimmune serum and $40 \mu \mathrm{l}$ of washed Pansorbin and incubated on a spinning wheel in cold room for 1 hour. After this pre-clearing, samples were centrifuged for $30 \mathrm{~min}$ at $13,000 \mathrm{RPM}, 4^{\circ} \mathrm{C}$. The supernatant was carefully transferred to new tubes, $3 \mu$ l of rabbit anti-TIP47 antibody were added to each tube and incubated on spinning wheel in cold room overnight for the formation of specific antigen-antibody complexes. $40 \mu$ of washed Protein-A agarose (50\% solution in PBS) were added and the incubation was continued for another one hour. The samples were centrifuged for $2 \mathrm{~min}$ at $13,000 \mathrm{RPM}, 4^{\circ} \mathrm{C}$. Supernatants were collected in fresh tubes and were frozen at $-20^{\circ} \mathrm{C}$. The pellets were washed five times with $\mathrm{NaCl}$-Tween20 solution and once with 1/10 PBS. $20 \mu$ l of LBS were added to each sample, boiled for $10 \mathrm{~min}$, centrifuged for $2 \mathrm{~min}$ at $13000 \mathrm{RPM}$. The solubilized proteins were separated by a $10 \%$ PAGE. 


\subsubsection{Immunoprecipitation of MPR46}

Solutions

TBS

Buffer A

TIN buffer

Immunomix (IMM)

Precipitation immunomix

(PIM)

Neufeld buffer
Working concentration

10 mM Tris-Cl, pH 7.4

$150 \mathrm{mM} \mathrm{NaCl}$

$0.1 \mathrm{M} \mathrm{NaAc}$

$0.2 \mathrm{M} \mathrm{NaCl}$

$0.5 \%$ TX100

50mM Imidazole $\mathrm{pH} 7.0$

$150 \mathrm{mM} \mathrm{NaCl} \&$ protease inhibitors

$1 \%$ Triton $\mathrm{X}-100$

$0.5 \%$ sodium deoxycholate

$10 \mathrm{mM}$ Sodium phosphate buffer, $\mathrm{pH} 7.4$

0.15 M Sodium chloride

$10 \% \mathrm{BSA}$ and $0.2 \%$ SDS in Immunomix with

protease inhibitors

10 mM Tris-Cl, $\mathrm{pH} 8.5$

0.6 M Sodium chloride

$0.1 \%$ SDS

$0.05 \%$ NP- 40

$2 \mathrm{M} \mathrm{KCl}$ in IMM

$2 \mathrm{M} \mathrm{KCl-IMM}$

Cells were metabolically labeled overnight as described earlier (3.2.3.2). After
labeling, cells were washed twice with cold PBS, scraped in $1 \mathrm{ml}$ PBS $(500 \mu \mathrm{l} \times 2)$ and centrifuged for $5 \mathrm{~min}$ at $3,000 \mathrm{RPM}, 4^{\circ} \mathrm{C}$. The supernatant was discarded, the pellet was resuspended in $400 \mu$ of TIN buffer, sonicated $3 \times 10$ s and incubated on vibrax in cold room for $20 \mathrm{~min}$ to solubilise all membrane proteins. After solublilisation, the samples were centrifuged at $13,000 \mathrm{RPM}, 20 \mathrm{~min}, 4^{\circ} \mathrm{C}$. The supernatant was transferred to a new tube and the insoluble pellet was discarded. To the supernatants, 0.8 volume of PIM, $3 \mu$ of rabbit pre-immune serum and $50 \mu \mathrm{l}$ of unwashed Pansorbin were added and incubated on a spinning wheel in cold room for 2 hours. After this pre-clearing of non-specifically interacting proteins, the samples 
were centrifuged at $13,000 \mathrm{RPM}, 10 \mathrm{~min}, 4^{\circ} \mathrm{C}$. Supernatant was carefully transferred to a new tube. $3 \mu \mathrm{l}$ of rabbit anti-MPR46 antibody was added to the samples and incubated in the cold room on the spinning wheel overnight. $40 \mu \mathrm{l}$ of washed ProteinA sepharose beads were added and the incubation was continued for two hours in the cold room on a spinning wheel. The antigen-antibody complexes bound to the beads were pelleted at 6,000 RPM, $5 \mathrm{~min}, 4^{\circ} \mathrm{C}$. Supernatant was discarded and the beads were washed with Neufeldbuffer, IMM, IMM containing $2 \mathrm{M} \mathrm{KCl}$, and 1/10 PBS. Washes were performed at 5,000 RPM, $4^{\circ} \mathrm{C}$ for $5 \mathrm{~min}$. After last wash, the supernatant was completely removed and $60 \mu \mathrm{l}$ of 1x Laemmli buffer with DTT was added and boiled for $5 \mathrm{~min}$ at $95^{\circ} \mathrm{C}$, incubated on ice for few seconds and centrifuged for 2 at 13,000 RPM. The proteins were resolved on a $10 \%$ SDS-PAGE (Minigels) at 100-125 $\mathrm{V}$ for about 2 hours (3.2.3.1.3). The gel was fixed in destaining solution for $20 \mathrm{~min}$, washed with water twice, $5 \mathrm{~min}$ each, dried in a BioRad gel drier and exposed onto a phosphoimager screen. The bands were visualized by autoradiography.

\subsubsection{Isolation of lipid droplets by subcellular fractionation}

Lysis buffer

$10 \mathrm{mM}$ Tris- $\mathrm{HCl} \quad \mathrm{pH} 7.4$

1 mM EDTA

containing protease inhibitors:

$40 \mu \mathrm{g} / \mathrm{ml}$ Aprotinin

$10 \mu \mathrm{g} / \mathrm{ml}$ Leupeptin

$1 \mu \mathrm{M}$ Pepstatin-A

Hela or TIP47 RNAi cells were plated onto $15 \mathrm{~cm}$ dishes $\left(45 \times 10^{4}\right.$ cells per dish) and grown $24 \mathrm{~h}$ in normal medium. Then the medium was aspirated, cells were supplemented with fresh medium with or without $600 \mu \mathrm{M}$ oleic acid and incubated for $12 \mathrm{~h}$. Cells were washed twice with cold PBS, scraped in PBS and pelleted by low speed centrifugation. Then they were disrupted by hyperosmotic shock according to an established procedure (Wolins et al., 2001). The cell pellets were dispersed by vortexing, concurrent with the dropwise addition of $2 \mathrm{M}(70 \%(\mathrm{w} / \mathrm{w}))$ sucrose dissolved in lysis buffer at room temperature. The cells were then incubated on ice for $10 \mathrm{~min}$, during which time they were vortexed for 30 s every 2 min. To maximize the osmotic 
shock, $3 \mathrm{ml}$ of lysis buffer were added rapidly while vortexing the sample. The homogenate was incubated for an additional $10 \mathrm{~min}$ on ice and vortexed for 30 s every $2 \mathrm{~min}$; the cells were then further disrupted by passing them through a 27-gauge needle four times.

Then lipid droplets (LD) were isolated essentially as described by $\mathrm{Yu}$ and coworkers (2000). The cell homogenate was centrifuged for $10 \mathrm{~min}$ at $1500 \mathrm{~g}$ at $4^{\circ} \mathrm{C}$ to pellet nuclei and intact cells. The supernatant was transferred to a 12-ml ultracentrifugation tube and overlaid sequentially with $2 \mathrm{ml}$ each of $0.27 \mathrm{M}$ sucrose in the lysis buffer, $0.135 \mathrm{M}$ sucrose in lysis buffer and lysis buffer itself as a top solution. Following centrifugation at $154324 \mathrm{~g}$ for $1 \mathrm{~h}, 7$ fractions of $1.5 \mathrm{ml}$ were collected from top to bottom: the buoyant LD (Nos. 1 and 2), the mid zone (No. 3) between LD and cytosol, and the cytosol (Nos. 4-7).

For analysis of the LD phospholipids and proteins, fraction No 1, containing most of the LD, was transferred into $50 \mathrm{ml}$ tube, mixed with $6 \mathrm{ml}$ methanol by vortexing and spin down 5000 RPM $1 \mathrm{~min}$. Then $1.5 \mathrm{ml}$ chloroform were added to the solution, mixed

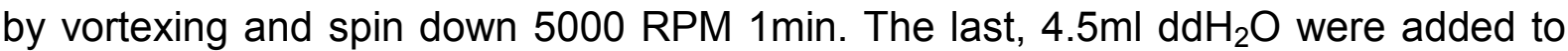
the mixture, vortexed for $5 \mathrm{~s}$ and centrifuged for $15 \mathrm{~min}$ at $5000 \mathrm{RPM}$ in order to separate phases. The upper phase was discarded, the lowest phase, containing extracted lipids, was directly subjected to mass spectrometry analysis, the proteins from the phase border were transferred into a new $1.5 \mathrm{ml}$ tube, dried in the air, resolved in $50 \mu \mathrm{L} \mathrm{LSB}$, boiled for $10 \mathrm{~min}$ and loaded on a SDS-PAGE (3.2.3.1.3). 


\subsection{Microscopy}

\subsubsection{Indirect Immunofluorescence}

\subsection{Fixing cells by Methanol}

Solutions

PBS

$10 \mathrm{mM} \mathrm{Na}_{2} \mathrm{HPO}_{4}, \mathrm{pH} 7.4,150 \mathrm{mM} \mathrm{NaCl}$

$10 \%$ goat serum $\quad 10 \%$ goat serum in PBS

Methanol

Cooled by storing at $-20^{\circ} \mathrm{C}$

DAKO

Mounting medium stored at $4^{\circ} \mathrm{C}$

The cells were plated on cover slips in a 24 well plate and grown till $50-70 \%$ confluency. Then they were washed twice with PBS. Solutions from the wells were sucked out using a tube connected to a vacuum pump and the following solutions were immediately added onto the cells. They were never let to dry up. The cells were fixed with methanol from $-20^{\circ} \mathrm{C}$ for 5 minutes. After fixation cells were washed 3 times with PBS at room temperature and incubated for $20 \mathrm{~min}$ at $4^{\circ} \mathrm{C}$. During this

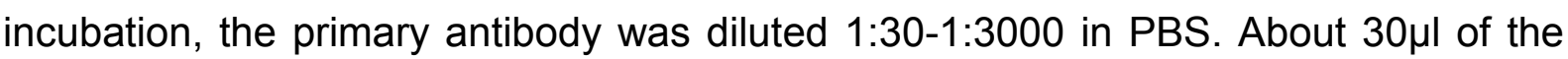
antibody solution was added on to each cover slip and incubated for $1 \mathrm{~h}$ at $37^{\circ} \mathrm{C}$. Care was taken not to disturb the plate during incubation with the antibodies. After incubation with the primary antibody cells were again washed 3 times with PBS and incubated $20 \mathrm{~min}$ with $10 \%$ goat serum to block non-specific binding. The cover slips were washed again twice with PBS and incubated for $1 \mathrm{~h}$ at $37^{\circ} \mathrm{C}$ with $30 \mu \mathrm{l}$ of $1: 50$ 1:200 diluted (in PBS) secondary antibody conjugated with a fluorescent dye (Alexa 633, Сy2, Сy3 or others depending on the experiment design). After this incubation, cells were washed two times with PBS and once with double distilled water. Meanwhile, clean glass slides were labeled and a drop of DAKO solution was added and kept aside. The cover slip was carefully taken out of the wells using syringe and a forceps, excess water was absorbed by gently touching it to a tissue paper and was placed on DAKO solution on the glass slide with the cell side facing the solution. These glass slides were incubated overnight at room temperature in dark. Next day, the border of the cover-slip was sealed with a nail-polish and after few minutes the 
cells were observed under fluorescence microscope. Slides were stored at $4^{\circ} \mathrm{C}$ for further analysis.

\subsection{Fixing cells by paraformaldehyde (PFA)}

Solutions

PBS

$10 \mathrm{mM} \mathrm{Na}_{2} \mathrm{HPO}_{4}, \mathrm{pH} 7.4,150 \mathrm{mM} \mathrm{NaCl}$

$3 \%$ PFA

$3 \%$ paraformaldehyde in PBS

The desired amount of PFA was weighed and a small amount of PBS was added, heated to $60^{\circ} \mathrm{C}$ on a heating block with constant mixing and after 10 min, 1-2 drops of $1 \mathrm{~N} \mathrm{NaOH}$ was added, solution gets cleared immediately and the volume was made up to the desired amount with PBS and the $\mathrm{pH}$ was adjusted to 7.4 with $\mathrm{HCl}$.

\section{PBS/Saponin $\quad 0.5 \%$ saponin in PBS}

DAKO Mounting medium stored at $4^{\circ} \mathrm{C}$

Cells were grown on sterile cover slips in a 24 well plate to $50-70 \%$ confluency, washed twice with PBS and fixed with 3\% PFA for $20 \mathrm{~min}$ at room temperature. The fixed cells were again washed twice with PBS. Cells were permeabilized with $0.5 \%$ Saponin in PBS for $3 \times 10$ min. Afterwards cells were washed twice with PBS and incubated with primary and secondary antibodies as well as with $10 \%$ goat serum as described above (3.3.1.1.1).

\subsubsection{Staining of LD with Nile red}

PFA fixation was found the best to preserve LD (DiDonato and Brasaemle, 2003) so it was used for further experiments. Nile red was reported as a selective fluorescent stain for the detection of intracellular lipid droplets by fluorescent microscopy (Greenspan et al., 1985a). Nile red was added to the secondary antibodies solution in dilution 1:1000.

\subsubsection{Transferrin endocytosis}

Transferrin is endocytosed from the medium by the transferrin receptor at the plasma membrane. The internalized transferrin is then delivered to the intracellular 
compartments and the transferrin receptor recycles back to the plasma membrane for further rounds of sorting. The kinetics of transferrin endocytosis and recycling has been well characterized and hence they are widely used as markers of the early and recycling endosomes.

HeLa and Cos cells were grown on coverslips to $50-60 \%$ confluency under standard cell culture coditions. The medium was replaced by a serum free medium containing $0.5 \%$ BSA (ultra pure) and incubated for further one hour. $0.1 \mu \mathrm{M}$ of Alexa633 conjugated mouse transferrin was added to the wells and mixed with a pipette for uniform concentration of transferrin in the medium and continued incubation at $37^{\circ} \mathrm{C}$ for $1 \mathrm{~h}$. This is the time required for the endocytosed transferrin to reach late endosomes. The cells were immediately shifted onto the ice and processed for immunoflorescence by PFA fixation as described in (3.3.1.1.2). The coverslips were incubated with polyclonal anti-TIP47 primary antibodies and FITC-conjugated antirabbit secondary antibodies. 


\section{$4 \quad$ Results}

\subsection{TIP47 localization}

\subsubsection{TIP47 detection in HeLa cells}

There were two reasons to thoroughly examine a number of proteins recognized by anti-TIP47 antibodies used in our experiments. On one hand, several proteins differing in molecular weight are recognized by an antiserum against pp17/TIP47, perhaps as a result of alternative splicing, as previously reported (Than et al., 2003; Than et al., 1998; Than et al., 1999). On the other hand, specificity of anti-TIP47 antibodies and localization of the protein in cells were a mater of a recent debate (Barbero et al., 2001; Wolins et al., 2001).

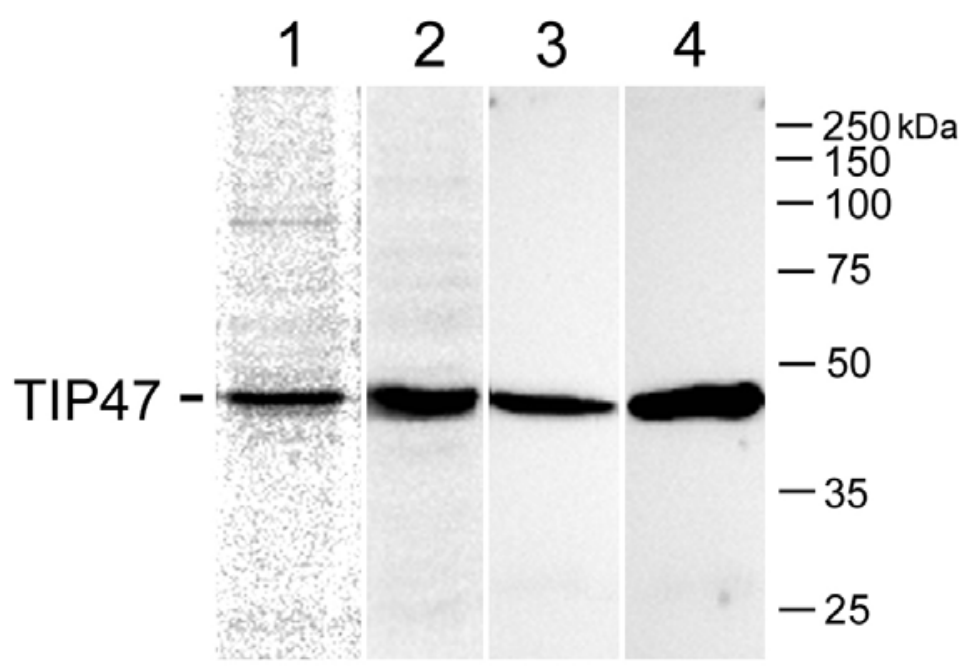

Figure 4.1. Specificity of TIP47 antibodies. Two antibodies from different sources were used in our experiments to detect TIP47 in whole HeLa cell lysates or immunoprecipitation. In lane 1 a single protein is visible, which was immunoprecipitated from metabolically labeled HeLa cells and has a molecular weight corresponding to the MW of TIP47. The same antibody was used to detect TIP47 via western-blotting in total cell lysates (lane 2). A band of exactly the same size was detected by another, commercially available antibody (lane 3), indicating that both Abs detect the same protein. This was further confirmed by using antibody 2 to detect the protein precipitated by antibody 1 . As shown in lane 4 , antibody 2 clearly recognizes the protein TIP47, which was first immunoprecipitated by antibody 1 , revealing that both antibodies are against the same protein.

In our experiments we used two anti-TIP47 antibodies: commercially available polyclonal antibodies (guinea pig) raised against a synthetic polypeptide corresponding to the N-terminal residues 1-16 of TIP47, and secondly, an affinity 
purified rabbit serum donated by S.R. Pfeffer (Barbero et al., 2001). Both sera were checked for their specificity on total HeLa cell lysates. As it is shown in Figure 4.1 both antibodies recognize the same protein (and only one) in western blotting as well as after immunoprecipitation. Thus, both antibodies could be used for further analysis of TIP47 in HeLa cells.

\subsubsection{Localisation of endogenous TIP47}

The distribution of endogenous TIP47 was first analyzed by immunofluorescence. Under standard cells culture conditions, TIP47 positive structures were not homogenously distributed among HeLa cells (Figure 4.2.). While some cells possess plenty of TIP47-positive structures, others exhibit moderate amount or no TIP47positive structures at all.

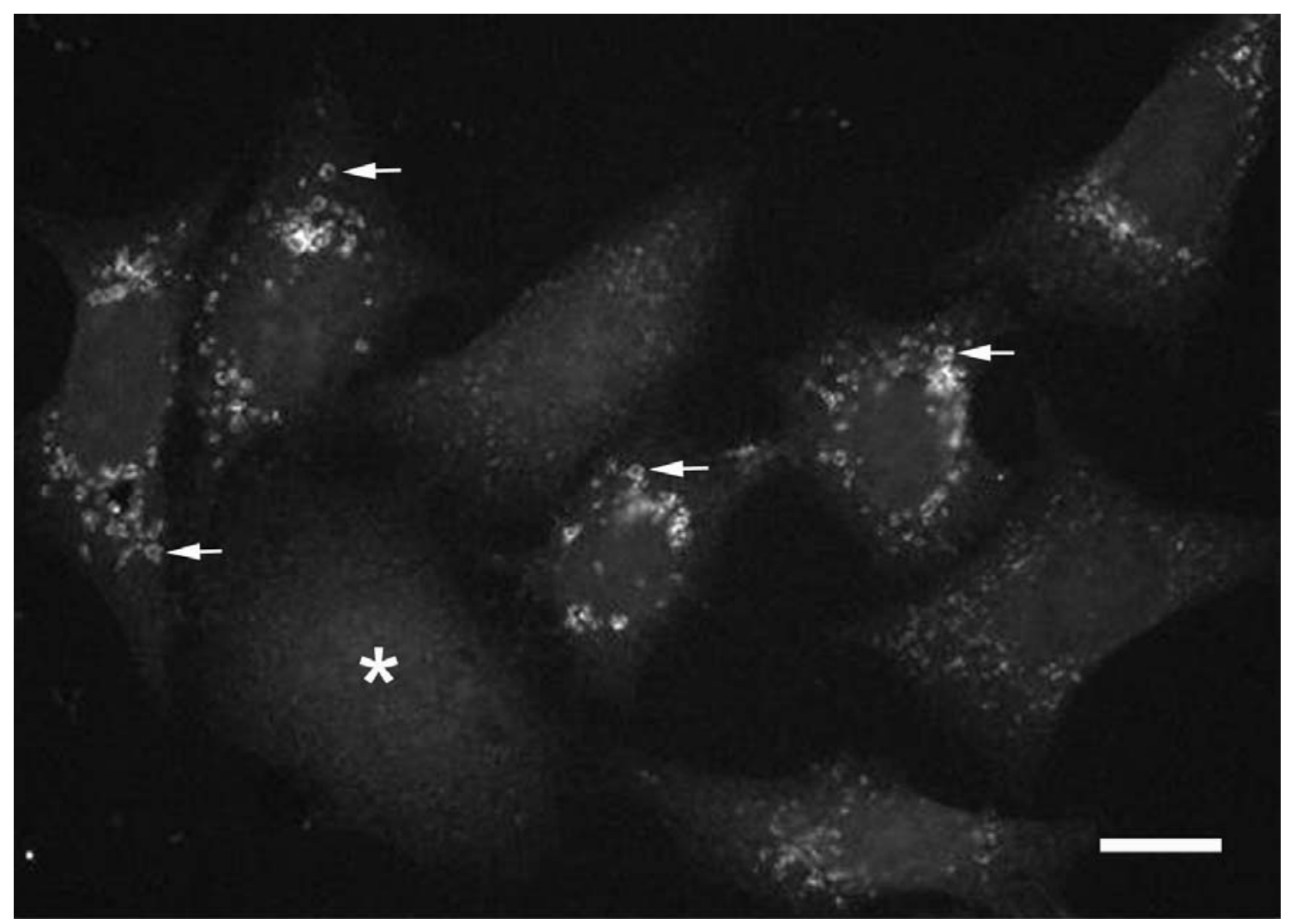

Figure 4.2. TIP47 distribution in HeLa cells. HeLa cells grown under standard cell culture conditions were fixed and further processed for immunofluorescence staining of TIP47. It was interesting to note that the distribution of TIP47 was not homogenous among all cells. While some cells exhibited a bright staining of TIP47, often found in "ring-like" structures (see arrows), other cells exhibited only a low degree of labeling and also some cells did not show any staining at all (see asterisk). Bar: $20 \mu \mathrm{m}$. 


\section{Cos cells}
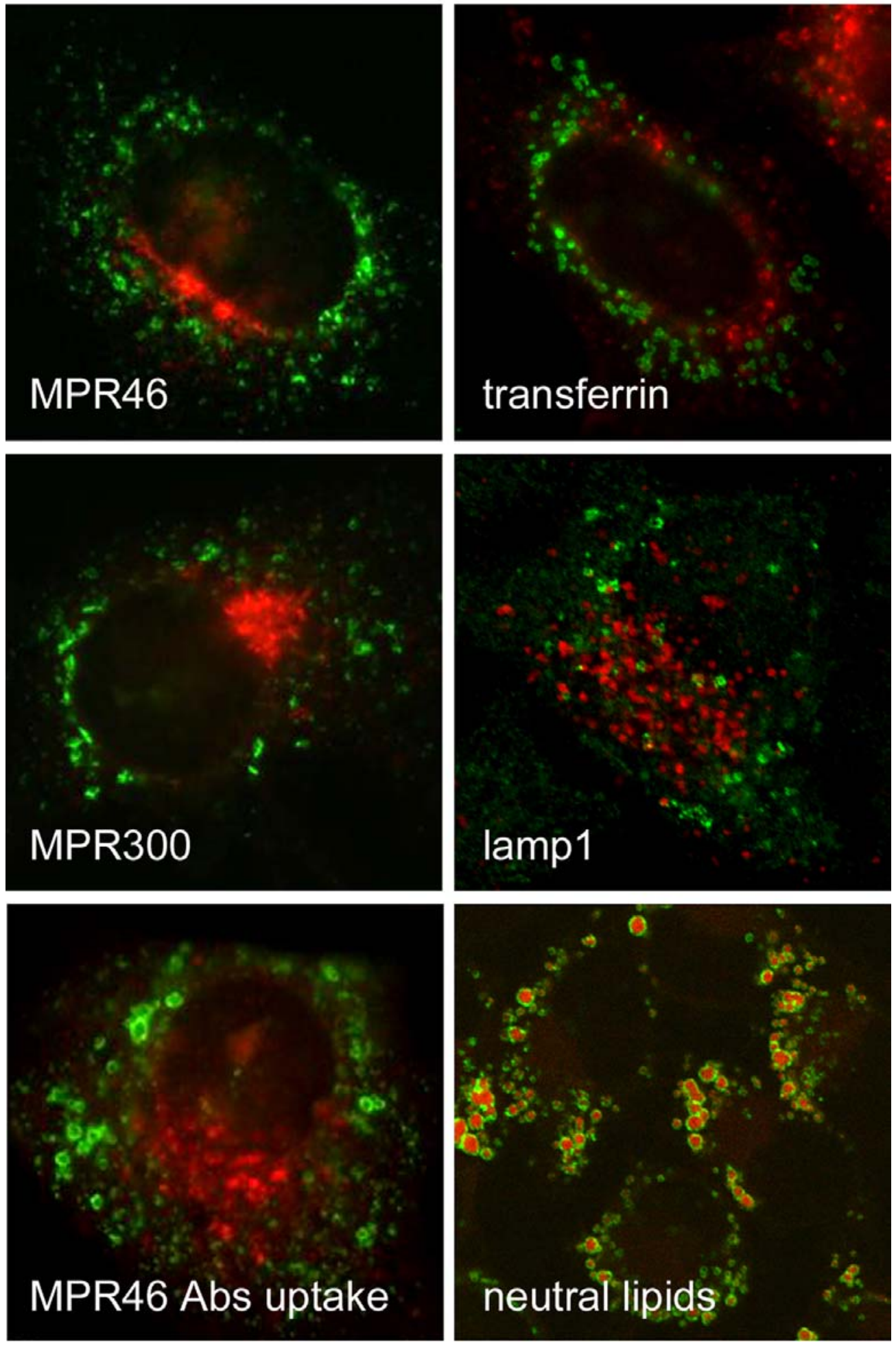

Figure 4.3. TIP47 does not colocalise with markers of the TGN, endosomes and lysosomes, but decorates the surface of lipid droplets in Cos and HeLa cells. Endogenous TIP47 is shown in green; MPR46, MPR300, lamp1, internalized transferrin and anti-MPR46 antibodies as well as neutral lipids stained by Nile red are shown in red. The distribution of each marker was tested in both cell lines and exhibited the same staining pattern. 
We next analysed the localisation of TIP47 to certain organelles in HeLa and Cos cells via immunofluorescence using compartment-specific markers. These experiments showed that TIP47 did not colocalise neither with endogenous mannose 6-phosphate receptors nor with internalized antibodies against mannose 6-phosphate receptors (Figure 4.3.). Furthermore, TIP47 could not be colocalised with endosomal (internalised transferrin) and lysosomal (lamp1) markers, but decorated the surface of the lipid droplets, which were stained with a specific dye for neutral lipids (Nile red).

\subsubsection{Localisation of tagged TIP47}

To further confirm the association of TIP47 with lipid droplets and to avoid any doubts according to the specificity of the TIP47 reactive antibodies (Barbero et al., 2001), we generated recombinant TIP47 tagged at the amino- and carboxy- termini. To this end, total mRNA was prepared from HeLa cells, followed by RT PCR with TIP47 specific 5'- and 3'-primers. The obtained coding region of the HeLa cell-derived TIP47 cDNA was fully sequenced to confirm its identity.

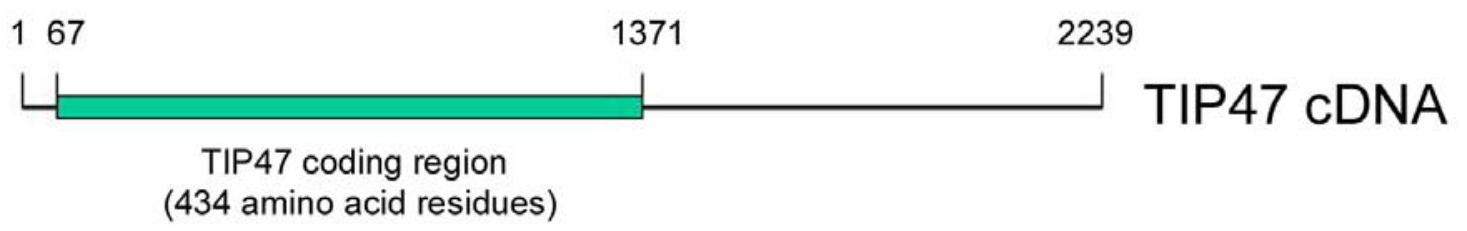

HA tag sequence TAT CCG TAT GAT GTG CCT GAC TAC GCA

RGS-His tag sequence AGA GGA TCG CAT CAC CAT CAC CAT CAC

Figure 4.4. Schematic representation of TIP47 cDNA and sequences of the HA- and RGS-His- tags used. Numbers indicate the total length of the cDNA as well as length of the translated sequence. Only the TIP47 coding sequence was amplified and cloned with HA- or RGS-His- tags on N- or Cterminus into the mammalian expression vector pMPSV-EH. The nucleotide sequence of the HA-tag corresponds to YPYDVPDVA polypeptide, and the RGS-His-tag (later referred as His-tag) to the sequence RGSHHHHHH.

Sequencing revealed that the cDNA cloned matches a published sequence of the cargo selection protein TIP47 deposited by Diaz, E.D. and Pfeffer, S.R. (accession number AAC39751), which is slightly different from the TIP47 sequence deposed by Strausberg, R. (accession number AAH05818) harboring a conservative substitution: lle56 was changed to Val. This change was independently observed by another 
group (M. Rudolf, Department of Structural Biology, Göttingen, personal communications).

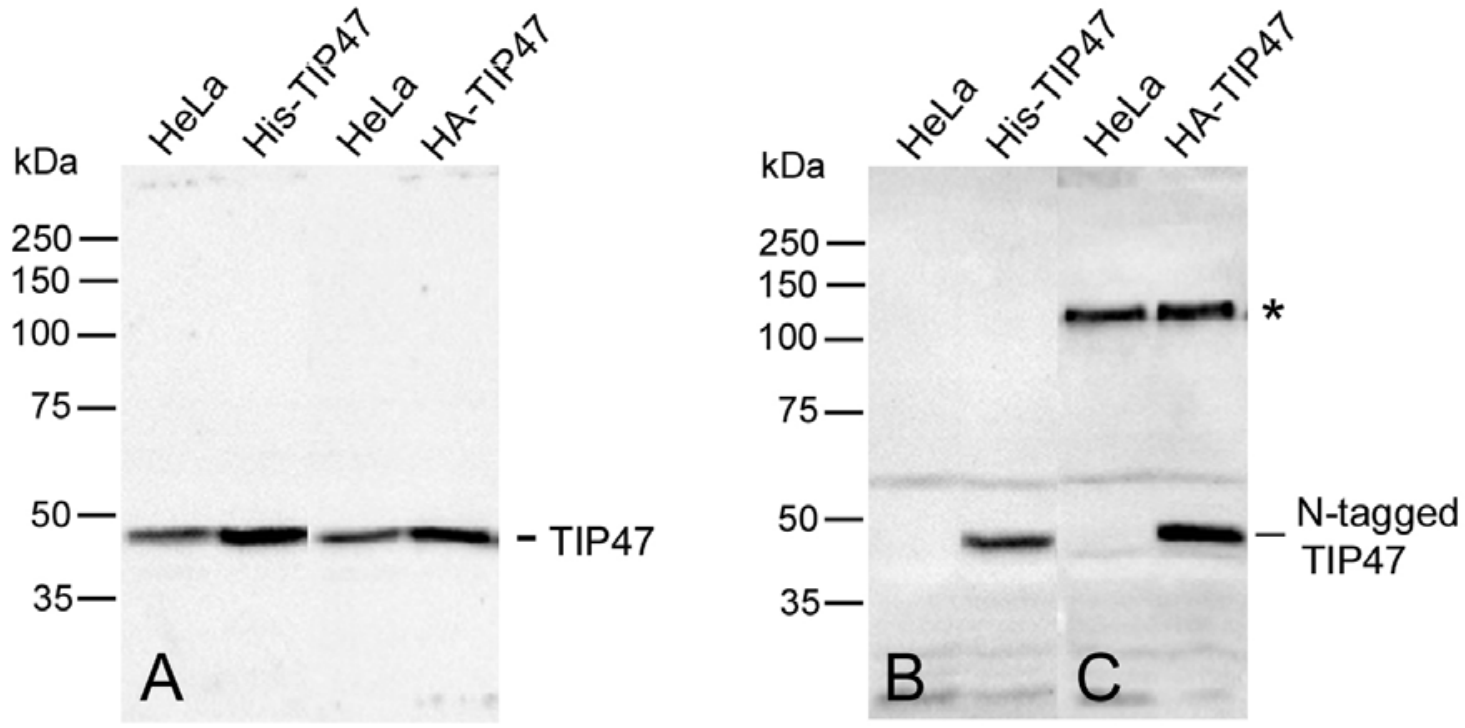

Figure 4.5. Lysates with equal amount of protein from untransfected and N-tagged TIP47 transfected HeLa cells were tested by Western blot analysis. In $\mathbf{A}$ the cell lysates were probed with rabbit anti TIP47 antibodies, in B with a monoclonal anti-His and in C with anti-HA antibodies. Both HA-and Histagged TIP47 comigrate with endogenous protein. The asterisk $\left(^{*}\right)$ in $C$ marks a protein which was unspecifically recognized by anti-HA antibodies.

The coding region of TIP47 cDNA was next used to generate tagged variants of the protein by fusing it either with the HA- or His- tag sequences in frame to the aminoor carboxy-termini (see 3.2.2.16 and Figure 4.4.). The derived tagged TIP47 was cloned into the pMPSV-EH vector and transiently expressed in HeLa cells under the control of the myeloproliferative sarcoma virus (MPSV) promoter (Artelt et al., 1988). Four days after transient transfection, total cells lysates of control HeLa cells and the transfected cells were prepared, subjected to SDS-PAGE, transferred onto nitrocellulose and tested with 3 antibodies: A) against TIP47, B) His- and C) HA-tags. As shown in Figure 4.5. a single band was labeled in transfected cells as well as in the control cells, when using a TIP47 antibody. Although one would have expected the tagged TIP47 to migrate at a slightly higher molecular weight, it could not be resolved in the Mini-gel system we were using. However, the extract of transfected cells exhibited a two-fold stronger signal, consistent with a moderate level of overexpression. Expression of the recombinant proteins was further confirmed by 
using HA- and His-tag specific antibodies, which only stained a protein of the expected size in the lysates of transfected cells.

In contrast to efficient detection of N-terminally tagged TIP47 in western blotting (see Figure 4.5.), the C-terminal tagged variants were hardly visible (not shown), although they were suitable for the detection of TIP47 in immunofluorescence (not shown). As a consequence, all further studies involving tagged TIP47 were performed with the amino-terminal tagged TIP47.

After having verified the expression of the recombinant TIP47 by western blotting we performed immunofluorescence to localize the tagged protein together with endogenous TIP47 and the MPR46. Both TIP47 and the tagged protein colocalised to almost $100 \%$, showing that the amino-terminal tag does not interfere with the localization of the fusion protein (Figure 4.6.). Moreover, the high degree of colocalisation demonstrated that the TIP47 specific antibodies stain the same structures, which are visualized by using tag-specific monoclonal antibodies. The experiment further revealed no colocalisation between TIP47 and MPRs, but staining of ring like structures that are identified as LDs by the incorporation of the lipophilic dye Nile red (see also Figure 4.26.).
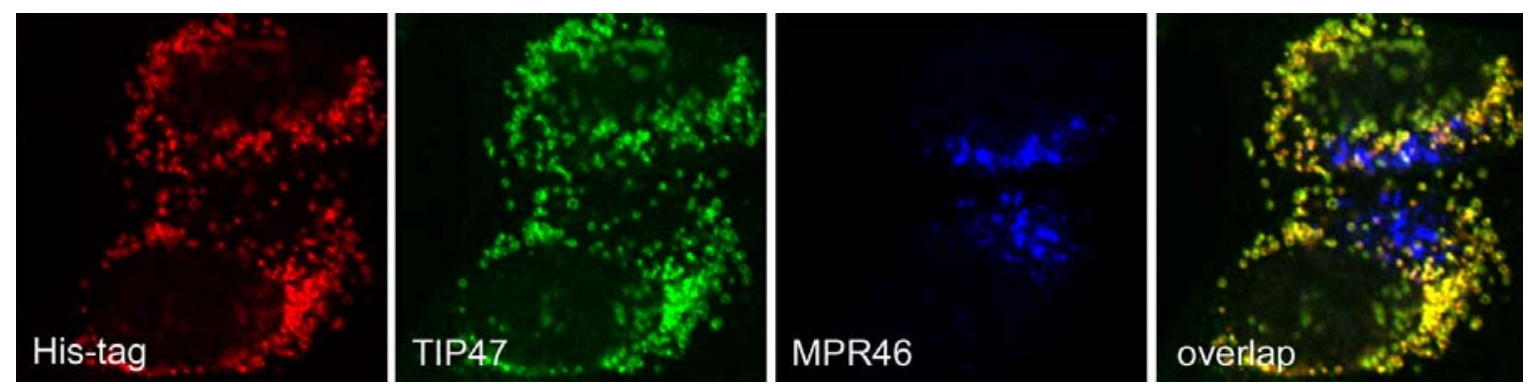

Figure 4.6. Tagged TIP47 does not colocalise with MPR46 in HeLa cells. Human TIP47 cDNA was cloned into the mammalian expression vector pMPSV-EH with His-tag and transiently transfected into HeLa cells. Two days after transfection, the cells were triple stained with rabbit anti-MPR46 (blue), guinea-pig anti-TIP47 (green) and mouse anti-His tag (red) antibodies and visualized with appropriate secondary antibodies. Endogenous TIP47 and the His-tagged protein exhibited colocalisation and were both well separated from the perinuclear staining of MPR46.

\subsubsection{TIP47 cofractionates with isolated LDs}

The association of TIP47 with lipid droplets was further revealed by an additional method well described in the literature - subcellular fractionation. After $12 \mathrm{~h}$ 
incubation with medium supplemented with $600 \mu \mathrm{M}$ oleic acid, the cells were harvested to prepare a postnuclear supernatant, which was further subjected to subcellular fractionation using a sucrose gradient. Indicative of LDs is that they float on top of such a gradient (Tauchi-Sato et al., 2002) from where they can be easily collected. As shown in Figure 4.7., a fraction of TIP47 could be detected in the floating material. The purity of the floating LDs was confirmed by blotting for other maker proteins, which were all well separated from the top fraction of the gradient. Thus, immunofluorescence as well as subcellular fractionation could unequivocally demonstrate the association of TIP47 with LDs.

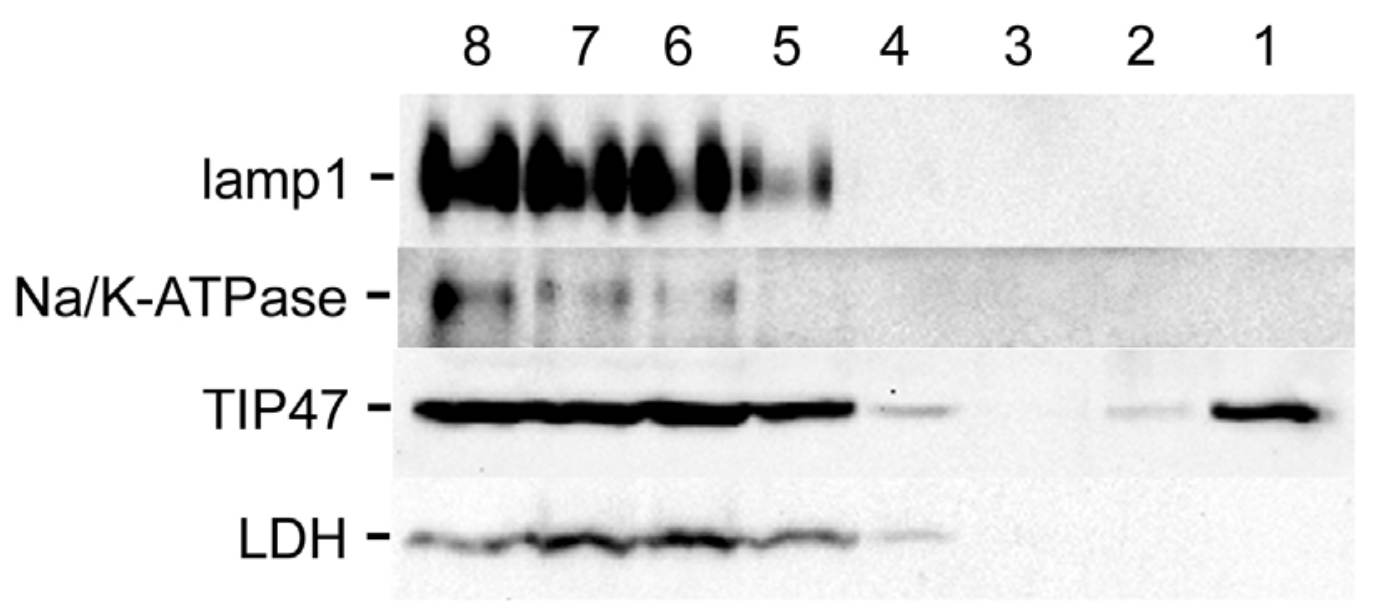

Figure 4.7. Endogenous TIP47 cofractionates with LDs in a sucrose gradient. HeLa cells were incubated $12 \mathrm{~h}$ in medium supplemented with $600 \mu \mathrm{M}$ oleic acid and then fractionated as described above (3.2.3.1.4). Aliquots of each gradient fraction were run on $10 \%$ SDS-PAGE, transferred onto nitrocellulose membrane and probed with antibodies against TIP47 and markers of several compartments: lactate dehydrogenase (LDH) - marker of the cytosol; lamp1 - late endosomes/lysosomes; Na/K-ATPase - plasma membrane. The lipid droplet fraction was well separated from the markers of other compartments and contained TIP47. The LDs are known to float on top of the gradient and are collected in fraction 1 . The western blot clearly showed that a fraction of TIP47 (16\%) is associated with floating LDs.

\subsubsection{TIP47 is not uniformly distributed on the LD surface}

The immunofluorescence showed that most often TIP47 covers not the whole surface of the LDs but concentrates to certain "domains" of the organelle (Figure 4.8.). This observation may suggest that the LD membrane is organized into microdomains and it may indicate that the LD membrane is of complex organization. 


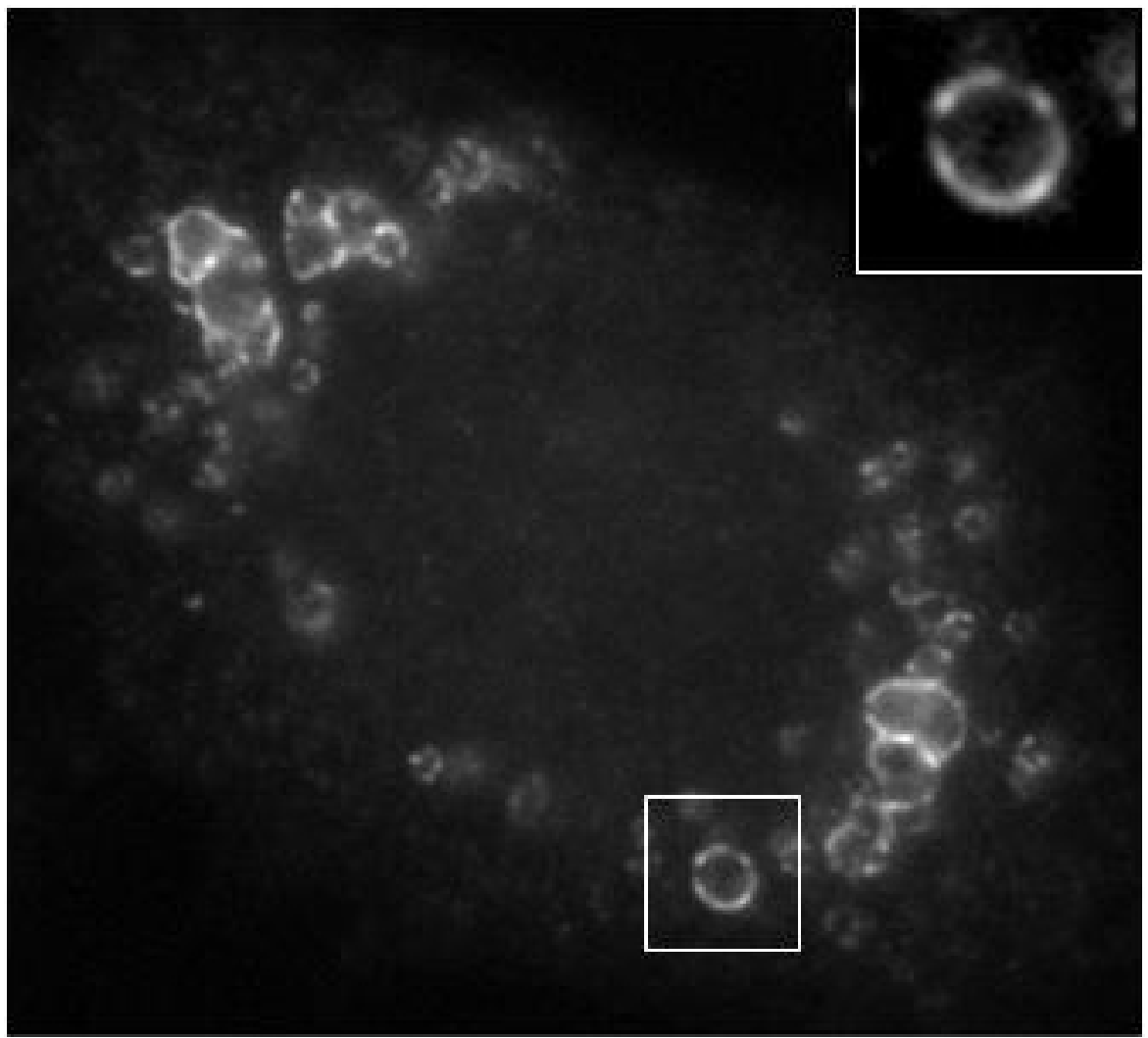

Figure 4.8. Distribution of TIP47 on the surface of lipid droplets. HeLa cell fixed with $3 \%$ PFA, permeabilised with $0.1 \%$ saponin and incubated with an antibody against TIP47 as described. Note that the protein is not randomly distributed and concentrated to certain "microdomains" (inset).

\subsubsection{Time course of TIP47 recruitment to the LD}

It was already shown by another group that the bulk of TIP47 is cytosolic and that upon accumulation of neutral lipids a fraction of the protein is recruited to the surface of the LDs (Wolins et al., 2001), however, nobody so far analysed the dynamics of TIP47 association with LDs. The time course of TIP47 recruitment to the organelles and its dependence on the nutritional status of the cell were studied in HeLa cells by conventional and quantitative immunofluorescence analysis. In order to estimate the fluorescence intensity of TIP47 positive LDs, 15 images were acquired for each time point in two independent experiments and the mean values of the fluorescence intensity were plotted.

We first analysed early events in LDs formation in HeLa cells preincubated overnight in starving medium (standard DMEM, supplemented with $2 \%$ FCS only). The preincubation leads to depletion of intracellular LDs as detected by Nile red and 


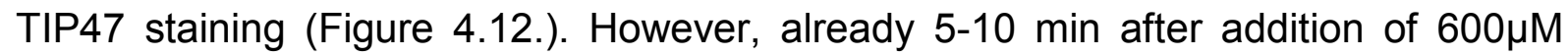
oleic acid to the culture medium, the first TIP47 positive structures with an average diameter of 100-200nm appeared (Figure 4.9.).
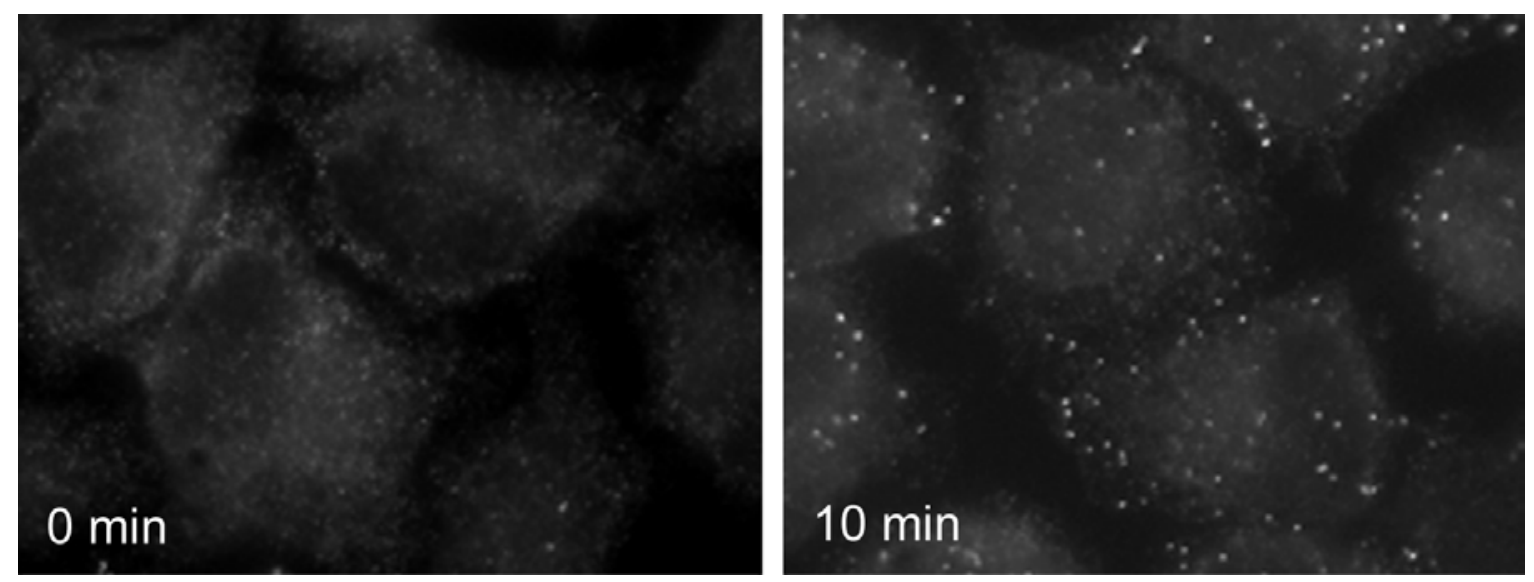

Figure 4.9. TIP47 translocates to the nascent lipid droplets early upon addition of oleic acid to the culture medium. HeLa cells were preincubated in presence of $2 \%$ FCS overnight and supplemented with DMEM containing 10\% FCS and 600 $\mu$ M oleic acid. First TIP47-positive structures were observed after 5-10 min incubation. The staining observed in the left image is background fluorescence that becomes visible because the two images were overexposed to show the small and relatively faint TIP47 decorated structures.

After longer periods of incubation, the quantity of the structures increased, they became larger in size and were later brightly stained with Nile red, as illustrated in Figure 4.10. Remarkably, the TIP47 staining of the nascent lipid droplets was maximal within 4-6h after administration of oleic acid, while the incorporation of Nile red was delayed by several hours (see graph in Figure 4.10., next page). 

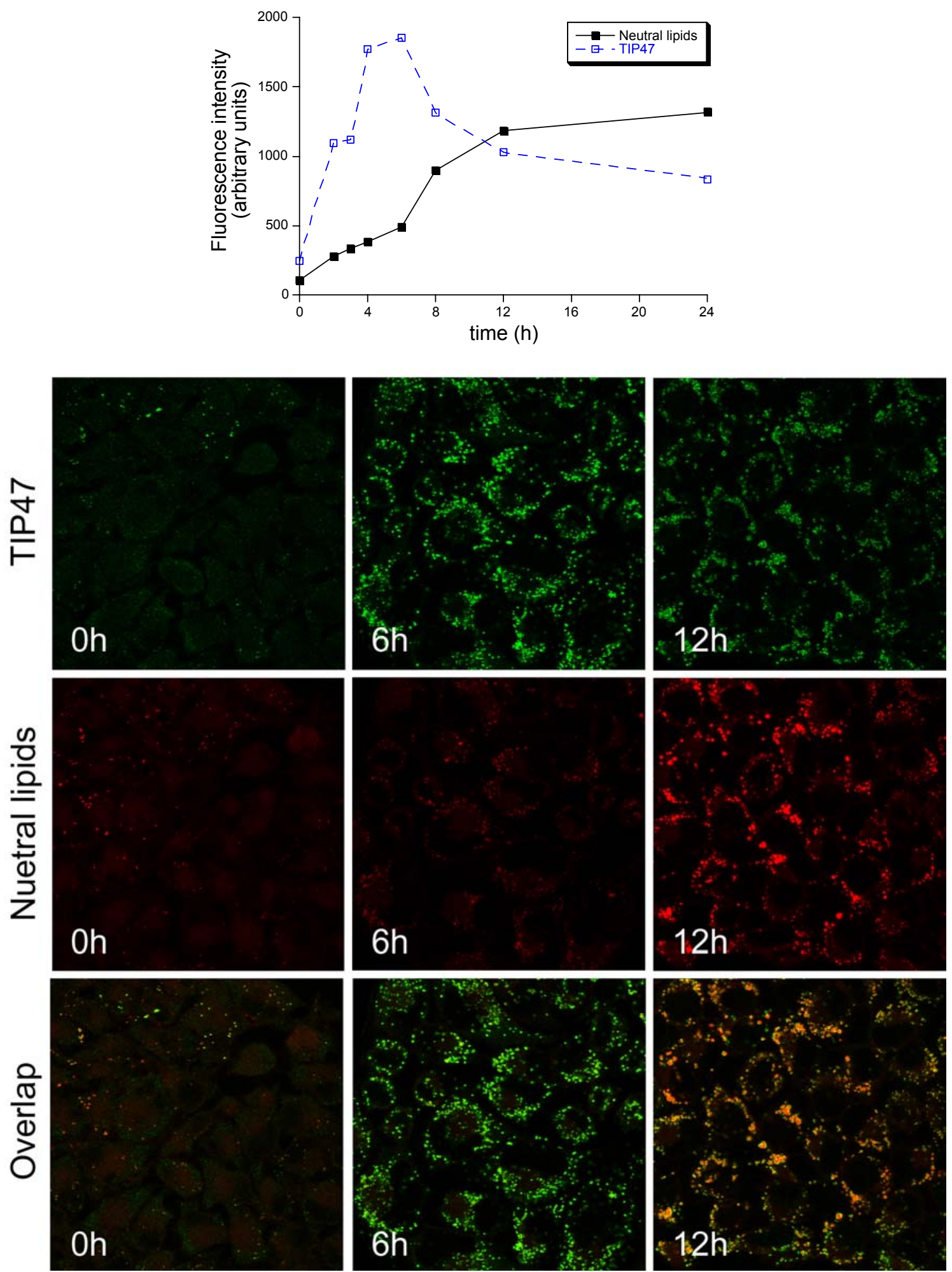

Figure 4.10. TIP47 recruitment to lipid droplets is maximal before the vast amount of neutral lipid is accumulated. HeLa cells were grown in normal medium (3.2.1.1) overnight and fixed or incubated in presence of $600 \mu \mathrm{M}$ oleic acid for 2, 3, 4, 6, 8, 12 or $24 \mathrm{~h}$ before indirect immunofluorescence (IIF) was performed. The quantitative data obtained by means of a Leica Confocal system are summarized in the graph, representative pictures are shown below. Note that the fluorescence intensity of TIP47positive structures (shown in green) reaches its half-maximum within 2-3h upon stimulation of LDs formation, while the accumulation of neutral lipids (as indicates Nile red staining, shown in red) is delayed to $7 \mathrm{~h}$ after feeding. 
The next aspect of interest was the influence of the nutritional status of a cell on TIP47 recruitment to membranes. Cells were incubated under starving conditions (2\% FCS) overnight before detection of TIP47 by immunofluorescence (Figure 4.11, A), or they were fed with $600 \mu \mathrm{M}$ oleic acid after starvation and then processed for IIF (Figure 4.11, B).

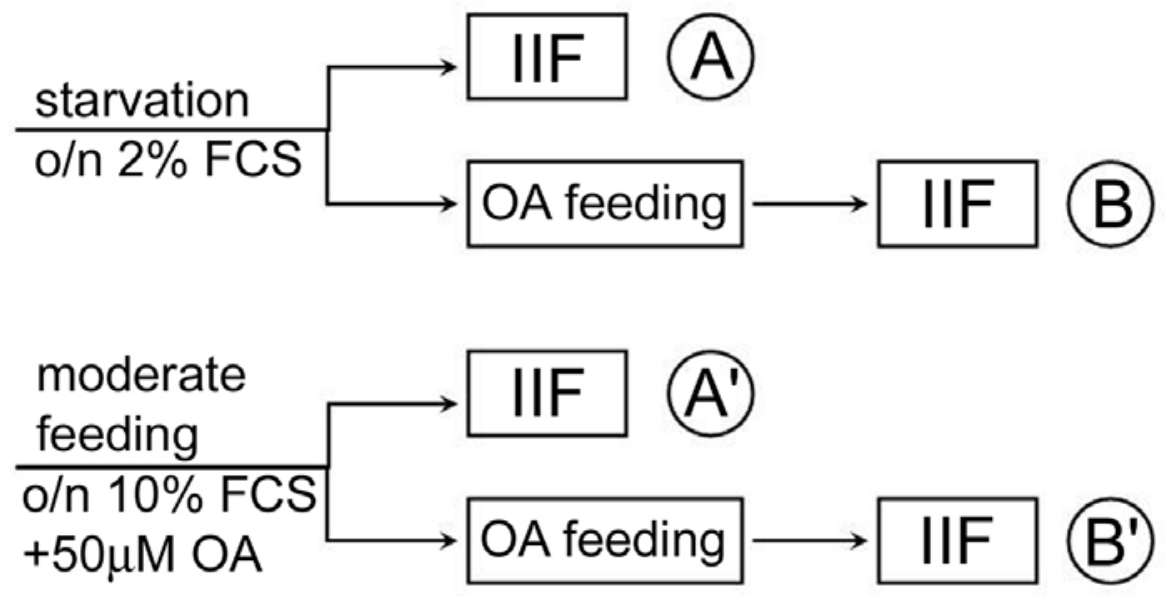

Figure 4.11. Influence of the nutritional status on recruitment of TIP47 to membranes. Outlined is the incubation protocol before TIP47 localisation was assayed by indirect immunofluorescence (IIF). Corresponding micrographs are shown in the next figure.

Alternatively, another batch of cells was incubated overnight under "moderate" feeding conditions (10\% FCS $+50 \mu \mathrm{M}$ oleic acid) before they were processed for TIP47 staining $\left(A^{\prime}\right)$, or they were further fed with $600 \mu \mathrm{M}$ oleic acid before TIP47 detection (B').

As shown in Figure 4.12., cells that were fixed immediately after starvation or moderate feeding both show a very low degree of TIP47 positive structures and almost no LDs as indicated by Nile red staining (compare $A$ and $A^{\prime}$ ). This similar pattern for TIP47 changed, when cells that were fed with oleic acid after starvation (Figure 4.12., B) are compared to those that received oleic acid after moderate feeding (Figure 4.12., B'). The later ones show a weaker TIP47 staining. This was also confirmed by a quantitative analysis of TIP47 fluorescence intensity (see graph in Figure 4.12.). Interestingly when cells were analysed that had been fed for longer periods of time $(>4 \mathrm{~h}$ ), the difference between starved and moderately fed cells became minimal, suggesting that only 


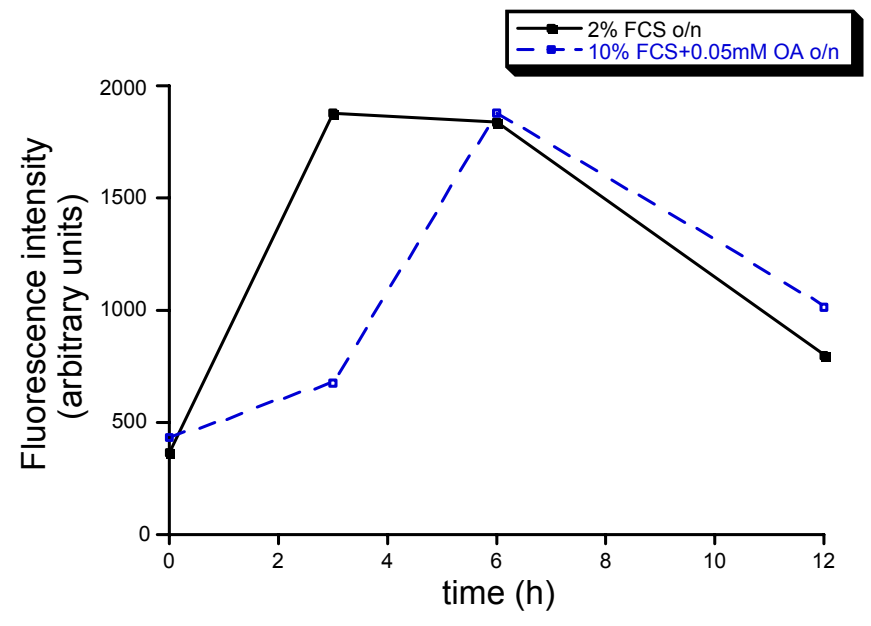

\section{$2 \% \mathrm{FCS}$ o/n}

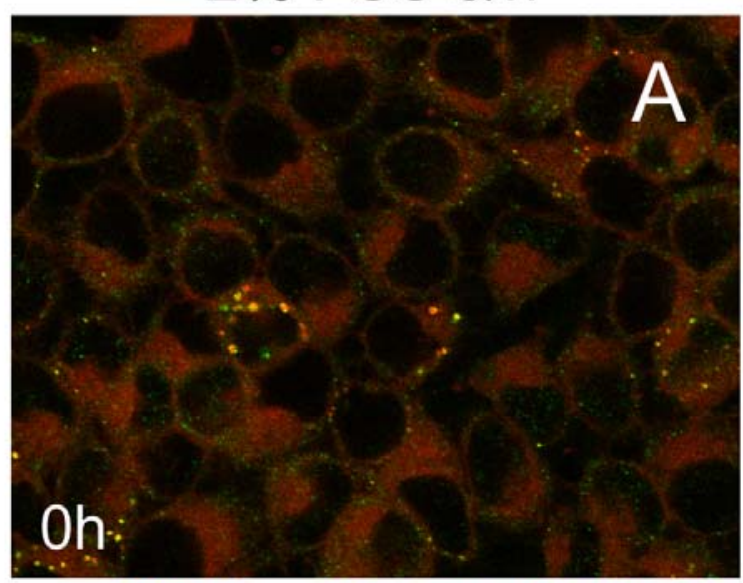

\section{$10 \% \mathrm{FCS}+50 \mu \mathrm{M}$ OA o/n}
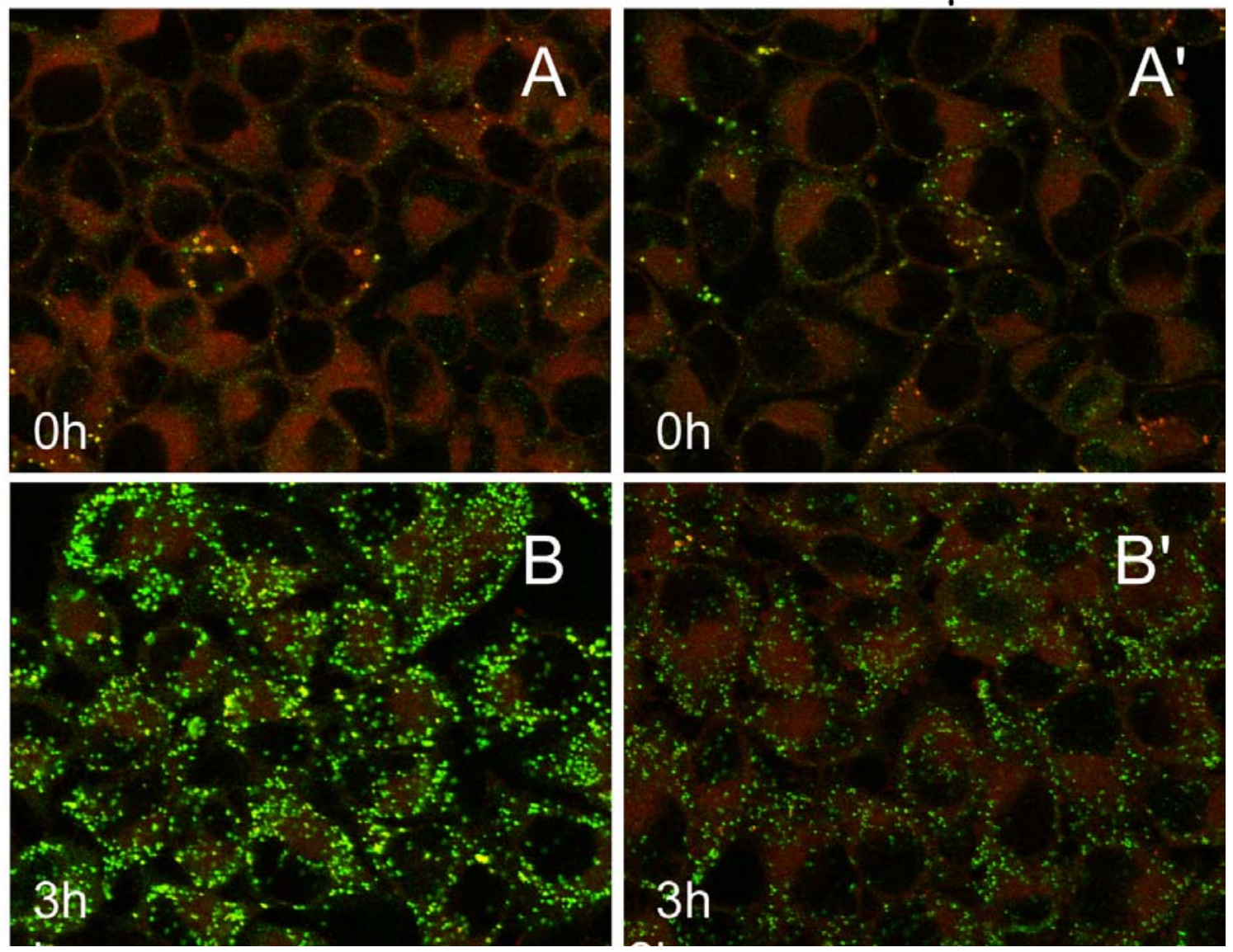

Figure 4.12. The time course of TIP47 recruitment to the lipid droplets depends on nutritional status of the cells. HeLa cells were incubated overnight in presence of $2 \%$ FCS or $10 \%$ FCS $+50 \mu M$ oleic acid, then supplemented with DMEM with $10 \%$ FCS and $600 \mu \mathrm{M}$ oleic acid and incubated 3, 6 or $12 \mathrm{~h}$, fixed and used for immunofluorescence analysis. The graph and photos above show that TIP47 translocates from the cytoplasm to the surface of the lipid droplets faster in starving cells and achieves its half-maximum in $1,5 \mathrm{~h}$, while in moderately prefeeded cells it occurs after $4 \mathrm{~h}$ incubation with $600 \mu \mathrm{M}$ oleic acid.

shortly after administration of fatty acids, the cellular response with respect to the recruitment of TIP47 to LDs is dependent on nutritional status. 
One could, for example, imagine that after feeding, TIP47 is primed somehow (phosphorylation or other posttranslational modification) to ensure rapid formation of new LDs after the cells receive new lipid. Alternatively, in a kind of negative feedback, feeding cells may lead to TIP47 modification that inhibit its rapid association with newly forming organelles. Another alternative is that not TIP47 by itself is the substrate that is modulated, but another factor associated with newly formed LD, which would mean that the effects on TIP47 are just secondary.

\subsection{Towards the function of TIP47}

The finding that TIP47 associates with the LD membrane early in the biogenesis of the organelle (see 4.1.6) is suggesting that LD formation involves the function of TIP47. However, TIP47 may also participate in the incorporation and/or exchange of material from the cytoplasm to LDs. As a starting point for a functional analysis, an RNAi approach has been chosen to suppress the expression of the protein, followed by the analysis of the affected cells.

\subsubsection{Establishment of a TIP47 RNAi cell line}

A TIP47 RNAi cell line was established as described in Materials and Methods (3.2.2.17). Shortly, two 19 nucleotide sequences flanked by AA-TT (starting at position 486 and 1435 of the full length TIP47 cDNA (shown in Figure 4.4) have been chosen to target human TIP47 mRNA by a plasmid based RNAi technique. The targeting sequence in direct and reverse orientation separated by a spacer (schematically shown in Figure 4.13.) were cloned into the plasmid pSHH, containing a U6 promoter and a neomycin resistance gene. 


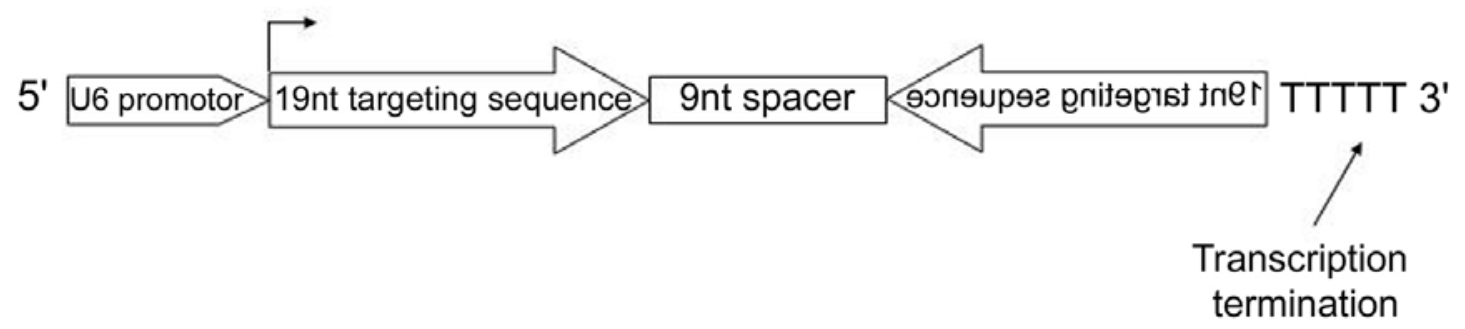

Figure 4.13. Schematic representation of the TIP47-specific primer sequence that generates a hairpin RNA in the cell. (From GeneSilenser ${ }^{\mathrm{TM}}$ Manual with modifications). Oligonucleotides were synthesized harboring the 19 nucleotides targeting sequences, a 9 nucleotide spacer and the targeting sequence in reverse orientation, flanked by a terminator of transcription (5T). They were cloned under the U6 small nuclear RNA promoter into the pSHH plasmid using Xhol and Xbal sites. The resulting vectors were stably transfected into HeLa cells in order to suppress the expression of TIP47.

The multicloning sites containing the RNAi constructs of the derived vectors were sequenced (3.2.2.18) followed by transfection into HeLa cells (3.2.1.5). HeLa clones resistant to $800 \mu \mathrm{g} / \mathrm{ml}$ neomycin were picked and propagated. The level of TIP47 expression was then tested by western blot (Figure 4.14.). Only one of the two targeting sequences, namely CCCGGGGCTCATTTCAAAC, starting at position 1435 in the 3' nontranslated region of the mRNA was efficient in suppressing the TIP47 expression level in HeLa cells. Clone number 1, in which $92 \%$ of expression were suppressed, was used in further experiments to elucidate the TIP47 function.

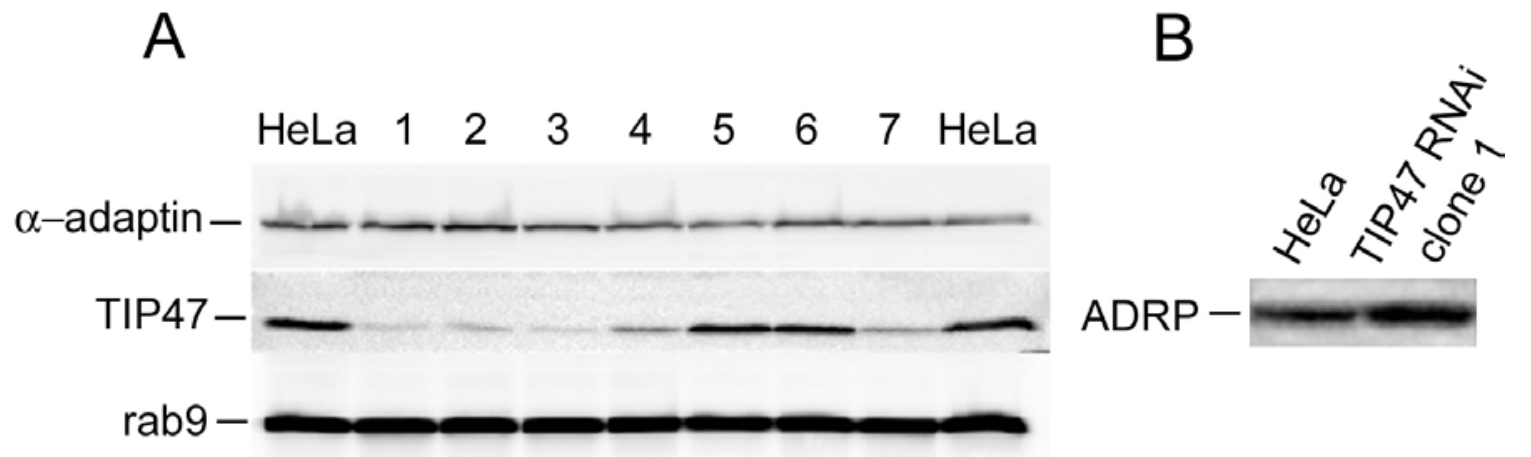

Figure 4.14. Lysates of control HeLa cells and TIP47 RNAi clones were analyzed by western blotting. $30 \mu \mathrm{g}$ of protein were loaded on each lane, electrophoretically separated and transferred onto nitrocellulose membrane. Primary antibodies used in the western blot are listed below. (A) Expression of TIP47 was efficiently and specifically suppressed in clones 1-3, while expression of other trafficking related proteins such as a-adaptin and rab9 was not affected. The different TIP47 RNAi clones are indicated with numbers. Clone number 1 , expressing only $8 \%$ of normal amounts of TIP47 was used for further experiments. (B) Importantly, the expression of ADRP, the most closely related protein to TIP47 (43\% identity) and associated with LDs - was not suppressed in TIP47 RNAi cells. 
It is worth to mention that suppression of TIP47 was specific, since the expression of other trafficking related proteins ( $\alpha$-adaptin, rab9) and adipophilin (associated with LDs) was not affected.

Interestingly, the remaining small amount of TIP47 that is left in TIP47 RNAi cells localized to the surface of the LDs in contrast to HeLa cells, where it is mostly cytosolic, as shown by immunofluorescence analysis (see Figure 4.18.) and subcellular fractionation (Figure 4.15.). The consequence of this finding is discussed later in the text (see 5.1.).

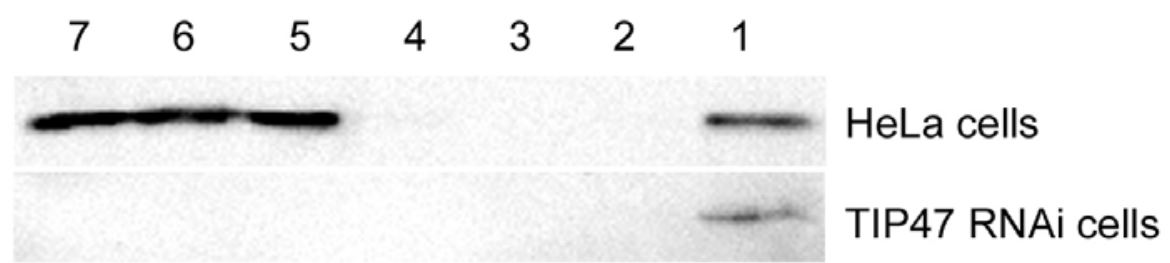

Figure 4.15. TIP47 is predominantly localized on the LDs in TIP47 RNAi cells. Control HeLa cells and TIP47 RNAi cells were incubated in presence of $600 \mu \mathrm{M}$ oleic acid for $12 \mathrm{~h}$ and subjected to subcellular fractionation in a sucrose gradient. Fractions (indicated with numbers) were collected as outlined in 3.2.3.1.4. In contrast to the control HeLa cells, where the bulk of TIP47 is cytosolic with $16 \%$ of TIP47 cofractionating with LDs, in the TIP47 RNAi cells all TIP47 is associated with LDs upon subcellular fractionation. It is important to note that the western blot of the RNAi material had to be overexposed in order to detect any TIP47 signal.

\subsubsection{Characterization of TIP47 RNAi cell line}

\subsubsection{MPRs transport is not affected by TIP47 RNAi}

Since TIP47 was supposed to play a key role in recycling of MPRs from late endosomes to the TGN (Diaz and Pfeffer, 1998), several aspects of MPRs trafficking in HeLa and TIP47 RNAi cells had to be examined. First, the depletion of TIP47 did not affect the steady-state distribution of MPR46 as detected by immunofluorescence (see Figure 4.16.). It was also observed that MPR46 did not accumulate in an endosomal/lysosomal compartment in TIP47 RNAi cells under normal cell culture conditions (Figure 4.16.) or in presence of a lysosomal protease inhibitor (leupeptin $100 \mu \mathrm{M}$, not shown). 

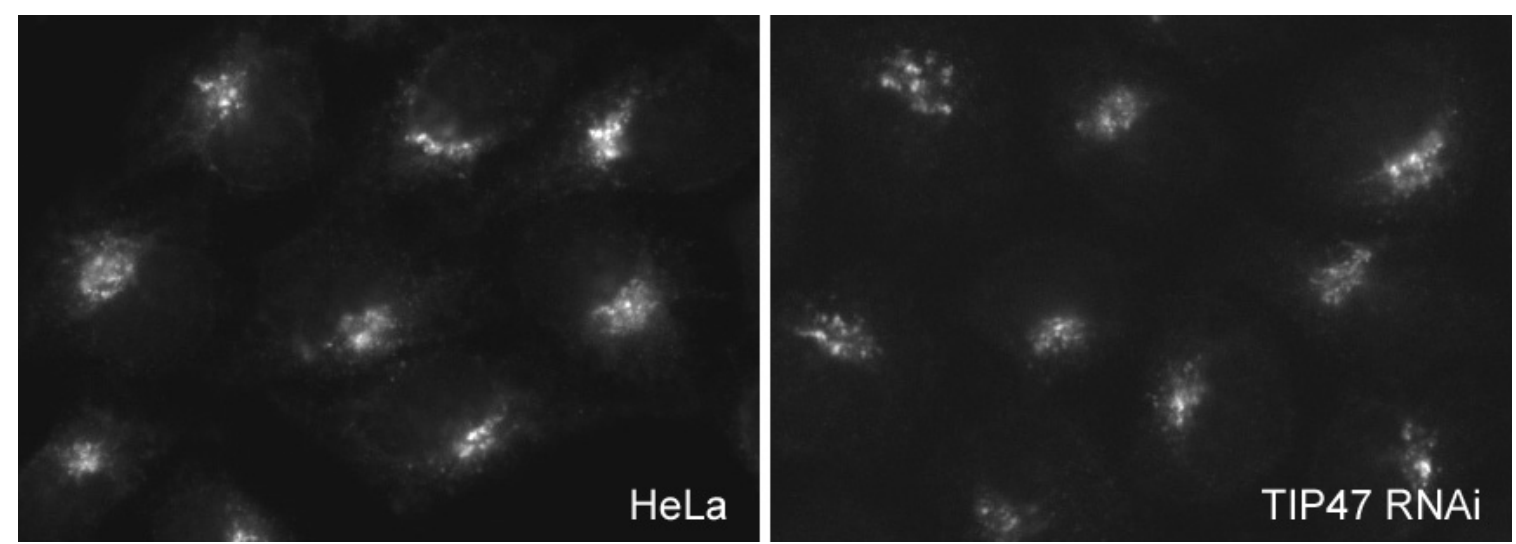

Figure 4.16. The steady-state distribution of MPR46 in TIP47 RNAi cells is not affected. Control HeLa cells and TIP47 RNAi cells were processed for immunofluorescence as described before. The staining pattern of MPR46 in RNAi cells shows no difference as compared to the control cells.

It is known that minor fractions of both MPRs are delivered to the plasma membrane, from where they are internalized and recycle back to the TGN. This transport can be visualized by an antibody uptake experiment during which antibodies that recognize the luminal domain of MPR46 or MPR300 are present in the medium. After 20min at $37^{\circ} \mathrm{C}$, cells were fixed and further processed to visualize the internalized antibodies. As shown in Figure 4.17. anti-MPRs antibody transport to the TGN is equally efficient in control and RNAi HeLa cells, suggesting that the trafficking of MPRs is not affected by suppression of TIP47.

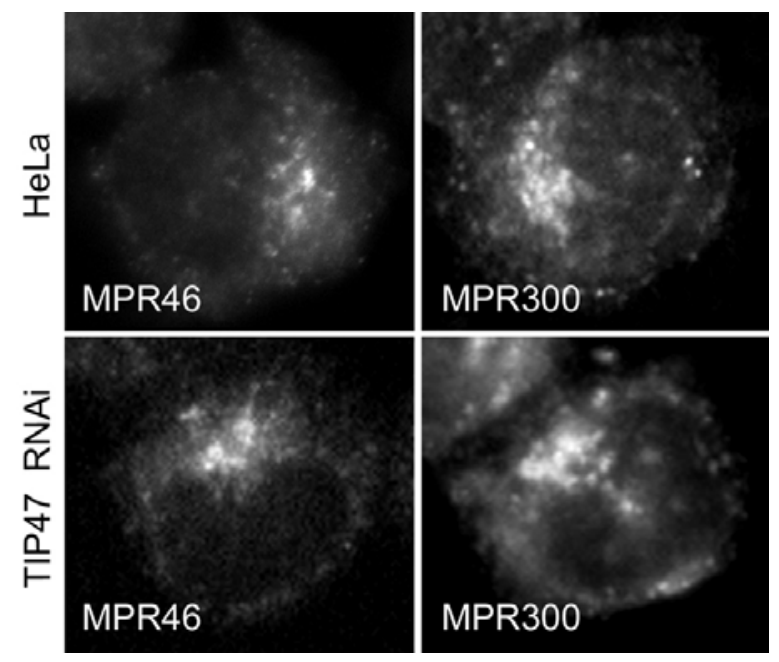

Figure 4.17. Internalized anti-MPRs antibodies are efficiently transported to the TGN region in both HeLa and TIP47 RNAi cells. Cells were incubated with anti-MPR46 or anti-MPR300 antibodies at $37^{\circ} \mathrm{C}$ for $20 \mathrm{~min}$, fixed, permeabilised and incubated with secondary antibodies to visualize the internalised primary antibodies. 
The half life of MPRs is strongly influenced by the efficiency of its retrieval from endosomes and transport back to the TGN (Rohrer et al., 1995). Therefore, we next tested the half life of MPR46 in HeLa and TIP47 RNAi cells in a pulse-chase experiment followed by immunoprecipitation of the receptor. Such experiments could not show any difference in the half life of the MPR46 between TIP47 RNAi and control cells (not shown). This result as well as the previous data strongly suggest that TIP47 has no role in the retrieval of MPRs from endosomes.

\subsubsection{The effect of TIP47 RNAi on LDs}

\subsection{LD morphology is altered by TIP47 RNAi}

As the previous results have shown the association of TIP47 with LDs it was now obvious to analyse the effect of the almost complete TIP47 suppression on various aspects of LD biogenesis, metabolism as well as the composition of the organelles.

A first observation was that the overall morphology of LDs formed in TIP47 RNAi cells after $12 \mathrm{~h}$ incubation in presence of $600 \mu \mathrm{M}$ oleic acid had changed in comparison to HeLa cells. The appearance of LDs was assessed by immunofluorescence, LDs were stained for TIP47 and neutral lipids (Nile red). 15 confocal images were taken for each cell line after two independent experiments to analyse the size, number and amount of LDs per cell. It turned out that the amount and size of LDs were affected. The RNAi cells exhibited LDs of very variable size as compared to the control cells and their number was reduced in many cells (Figure 4.18.). This may indicate that either TIP47 is involved in the formation of the organelles but it could also point to function of TIP47 in the size-control of LDs. 


\section{HeLa cells}

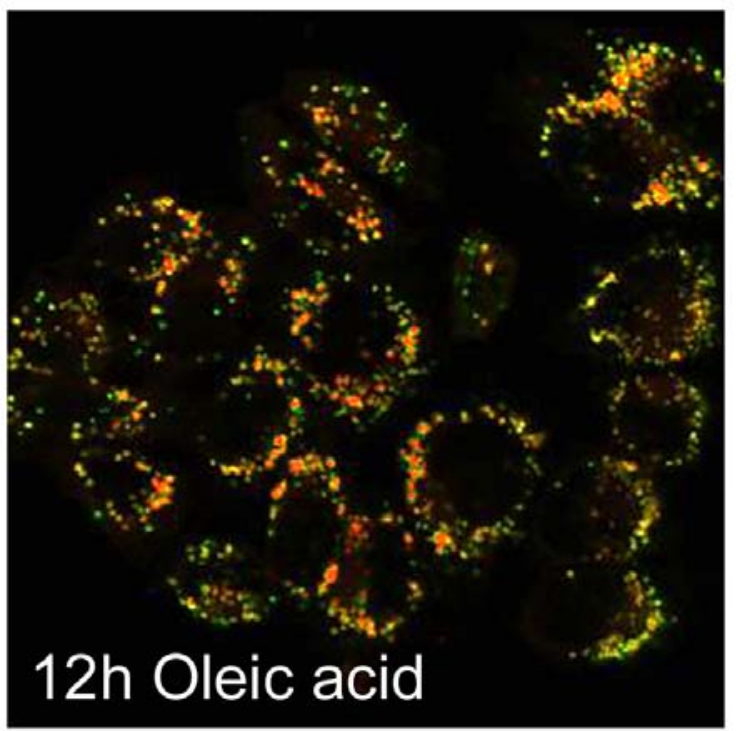

TIP47 RNAi cells

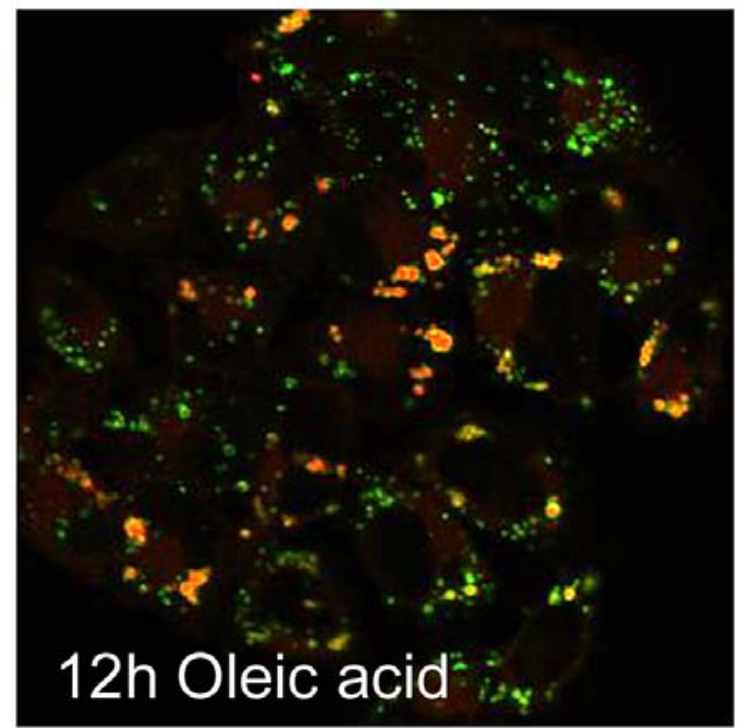

Figure 4.18. TIP47 RNAi cells form less uniform LDs than HeLa cells after $12 \mathrm{~h}$ incubation in medium supplemented with $600 \mu \mathrm{M}$ oleic acid. The LD population formed by TIP47 RNAi cells is less uniform with respect to the organelle size and the LDs were less abundant than in the control. TIP47 is shown in green, neutral lipids are shown in red.

\subsection{Fatty acid uptake and incorporation into LDs}

Long chain fatty acids are efficiently incorporated into triacylglycerols (Guo et al., 2000), which are the major components of LDs in many cells. As these fatty acids are mostly imported from the cellular exterior we next elucidated whether TIP47 RNAi had any effect on the uptake of long chain fatty acids and their incorporation into LDs. Three species of ${ }^{3} \mathrm{H}$ - or ${ }^{14} \mathrm{C}$-labeled fatty acids, saturated (palmitic), monounsaturated (oleic) and polyunsaturated (arachidonic) were added to the cells under three experimental conditions:

a) Radioactively labeled fatty acids were added to cells in suspension at a concentration of $20 \mu \mathrm{M}$ in PBS to estimate the efficiency of the fatty acids uptake over short time periods (5-30 min);

b) Radioactively labeled fatty acids were added to adherent cells at $20 \mu \mathrm{M}$ in the medium and incubated for periods ranging from $10 \mathrm{~min}$ to $12 \mathrm{~h}$;

c) Radioactively labeled fatty acids were diluted with the corresponding unlabeled fatty acids and added to the standard cell culture medium at $600 \mu \mathrm{M}$ and incubated with the cells for up to $8 \mathrm{~h}$. 

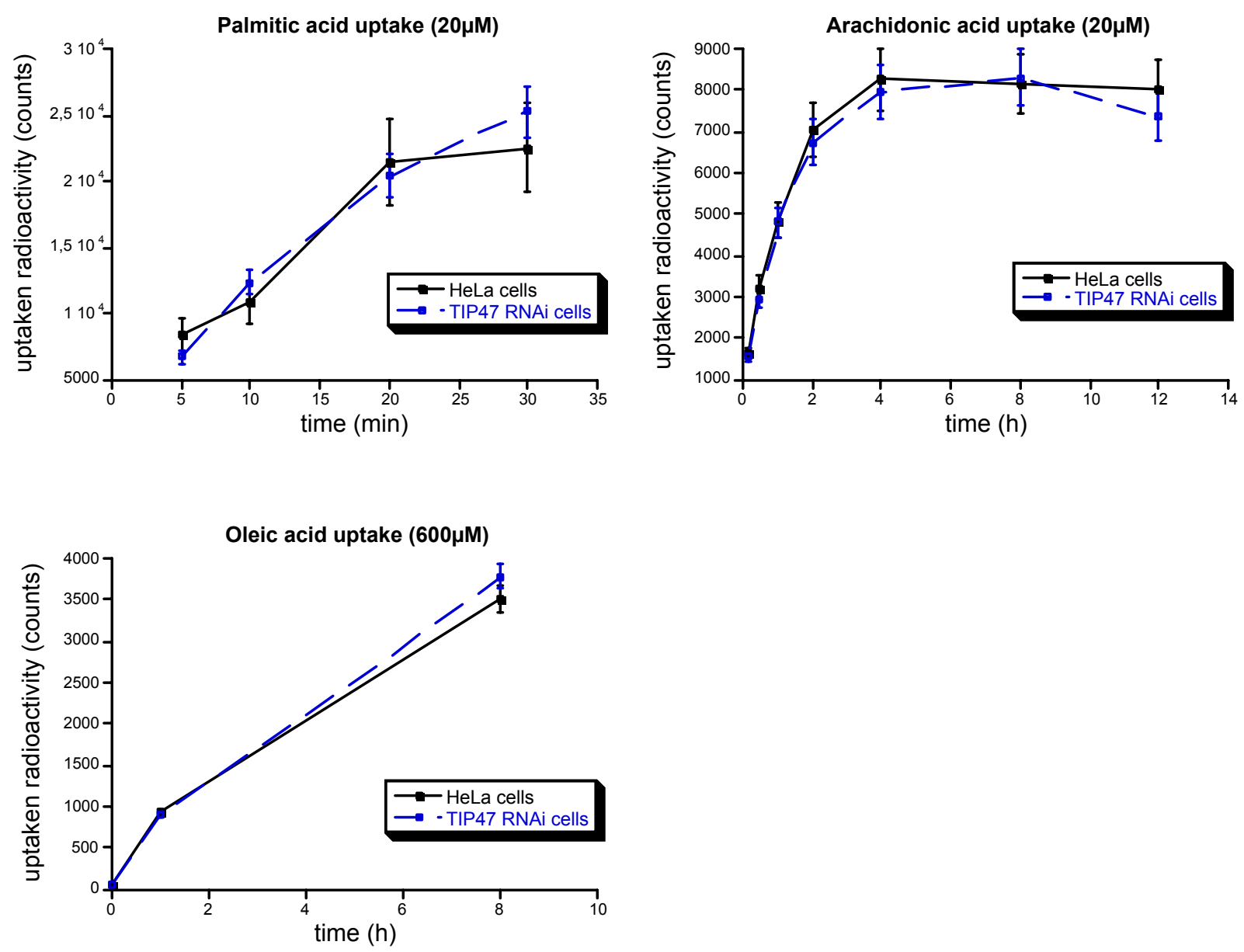

Figure 4.19 HeLa and TIP47 RNAi cells take up equal amounts of long chain fatty acids from the medium. The uptake of palmitic (saturated), oleic (mono-unsaturated) and arachidonic (polyunsaturated) acids in concentrations of $20 \mu \mathrm{M}$ or $600 \mu \mathrm{M}$ was monitored for different periods of time. All experiments were performed in duplicates (with oleic acid) or in triplicates (with palmitic and arachidonic acids), the incorporated radioactivity was normalized to the amount of protein.

After the incubation with fatty acids the cells were washed twice with PBS, harvested and homogenized, followed by determination of protein concentration and counting of the amount of incorporated radioactivity.

All these experiments showed that TIP47 RNAi cells take up normal amounts of either saturated or unsaturated long chain fatty acids under all experimental conditions tested.

As it was mentioned before, long chain fatty acids taken up by cells serve as substrates for synthesis of triacylglycerols, stored in LDs. After having shown that the uptake of fatty acids is not affected by TIP47 RNAi we next analysed the appearance of the radioactively labeled fatty acids in LDs. The LDs were purified by subcellular 
fractionation as described above and analysed for incorporated radioactivity. In contrast to the unaffected uptake of fatty acids into the cells these experiments revealed $50 \%$ reduction in the incorporation of radiolabeled oleic acid into LDs (Figure 4.20). However, the current data does not allow to draw the conclusion that TIP47 is directly involved in the incorporation of radiolabeled material into LDs.

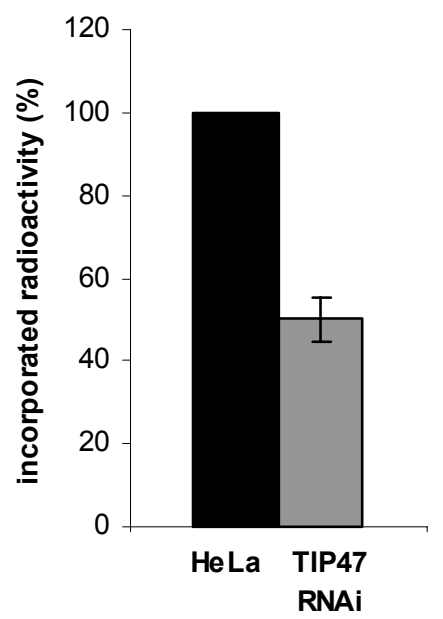

Figure 4.20. Oleic acid incorporation into LDs of TIP47 RNAi cells is reduced. HeLa and TIP47 RNAi cells were grown for $24 \mathrm{~h}$ and then incubated during $12 \mathrm{~h}$ in presence of $600 \mu \mathrm{M}$ cold oleic acid and $200 \mathrm{nCi}$ of ${ }^{14} \mathrm{C}$-oleic acid, harvested and fractionated. The amount of radiolabeled oleic acid in LDs was normalized to the cellular protein and plotted. The standard deviation of three experiments is shown.

\subsection{Phospholipid and protein composition of lipid droplets}

The loss of TIP47 expression may not only cause subtle effect on the incorporation of certain contents into the organelles, it is of course also possible that the overall LD associated protein and/or lipid composition is changed. To search for such changes, cells were incubated in presence of $600 \mu \mathrm{M}$ oleic acid for $12 \mathrm{~h}$ to induce LD formation. Subsequently, the cells were harvested and subjected to subcellular fractionation to collect the LDs. The associated lipids and proteins were separated by methanolchloroform extraction (see 3.2.3.1.4). A fraction of the extracted lipids was then directly applied to Maldi-MS. Such analysis only allows to detect major changes in the phospholipid composition while subtle changes in single species of phospholipids as well as other lipids will not be identified. As shown in Figure 4.21. the Maldi-MS spectra look almost identical, indicating no major changes in the phospholipid composition of LDs from TIP47 RNAi cells. I of course aware of the fact that only a 
more sophisticated MS analysis is needed to elucidate effect on single phospholipid species, which cannot be ruled out by the method used.

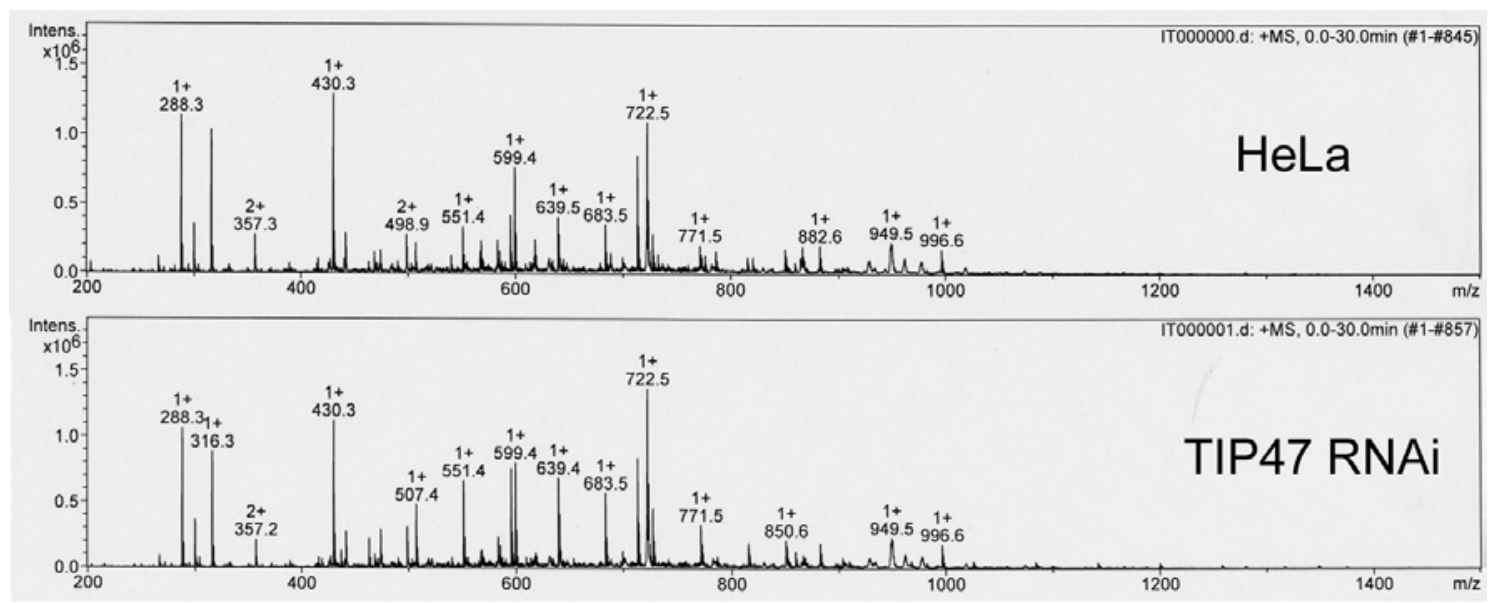

Figure 4.21. No major differences were observed in the phospholipid composition of isolated lipid droplets of HeLa and TIP47 RNAi cells by means of Maldi-MS. LDs isolated from TIP47 RNAi and HeLa cells by sucrose gradients were subjected to methanol-chloroform extraction. The derived lipids were collected and used for Maldi mass spectrometry.

The extracted proteins were separated by SDS-PAGE and silver stained (Figure 4.22.). Only one major difference in the protein pattern between control and TIP47 RNAi cells was detectable, - a substantial reduction of a protein with a molecular weight below 50kD. MS-analysis identified this protein as TIP47 (see A in Figure 4.22). This result also confirms the specificity of the RNAi since only the target protein is affected in its expression. 
Another unexpected finding was that TIP47 belongs to the most prominent bands of the resolved LD associated proteins. Very recently, other groups have also started to analyse the proteom of LDs (Athenstaedt et al., 1999; Liu et al., 2003). In contrast to the attempt of Anderson and coworkers (2003) who washed their purified LDs very extensively, our LDs derived from subcellular fractionation were directly subjected for SDS-PAGE. Interestingly, TIP47 is not associated with such extensively washed LDs, suggesting that washing is useful to reduce some contaminating proteins but also harbors the risk of loosing proteins only weakly associated with the LD membrane.
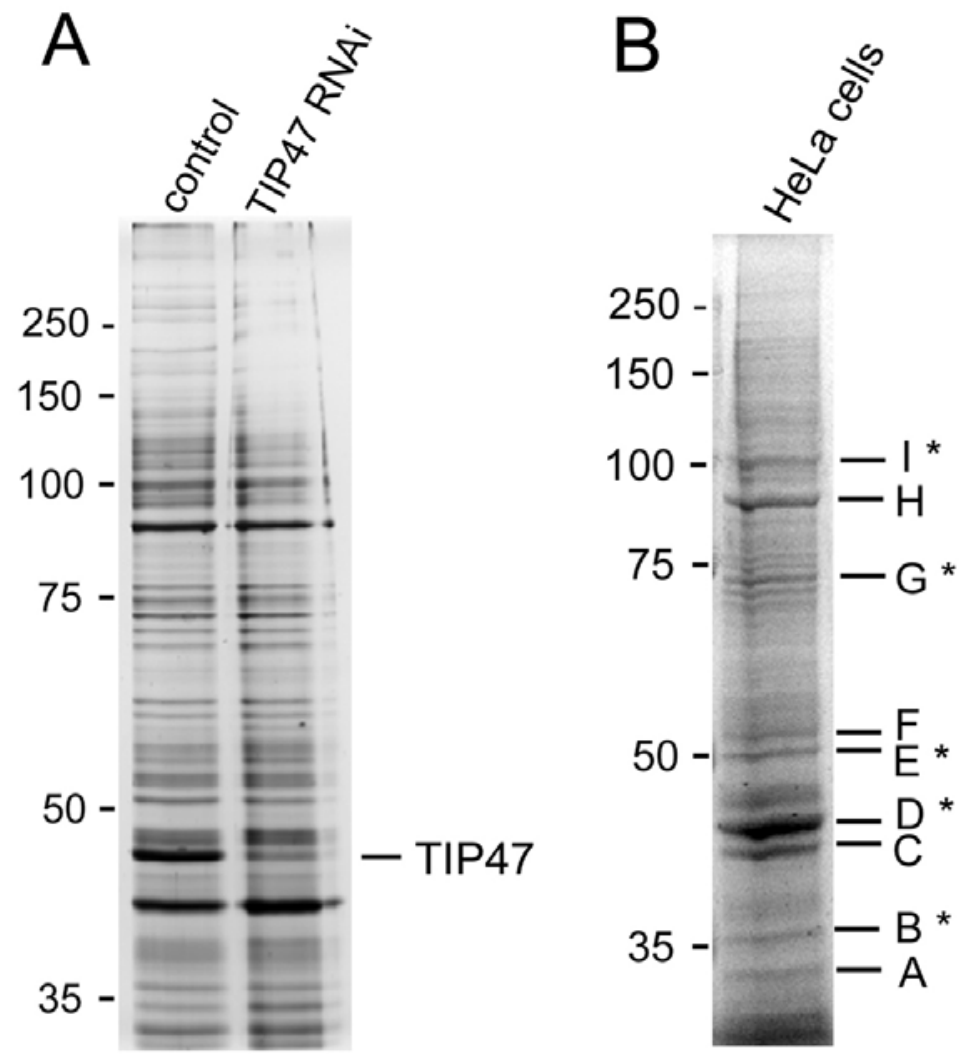

Figure 4.22. (A) TIP47 RNAi does not alter the typical LD protein composition. LDs collected after subcellular fractionation were extracted with methanol-chloroform and then subjected to SDS-PAGE. Equal amounts of protein were loaded. TIP47, one of the major proteins visible in the control LDs is reduced to a faint band in RNAi cells, showing the specificity and efficiency of the approach. (B) LDs of $615 \mathrm{~cm}$ dishes HeLa cells were collected, resolved by SDS-PAGE and stained with coomassie. Some of the protein bands were subjected to Maldi-MS identification. Letter A indicats glyceraldehyde3-phosphate dehydrogenase, $B$ - NAD $(P)$ dependent steroid dehydrogenase like protein, $C$ - $\beta$-actin, $D$ - TIP47, E - adipophilin, F - tubulin $\beta 5, \mathrm{G}$ - fatty acid (long chain) CoA ligase $3, \mathrm{H}$ - heat shock protein 1- $\beta$ of $90 \mathrm{kD}, \mathrm{I}$ - cytosolic calcium-dependent phospholipase A2 (group IVA). Asterisks $\left({ }^{*}\right)$ mark known LD associated proteins. 
A Coomassie blue stained SDS-PAGE of HeLa derived LDs is shown in Figure 4.22. B. From this gel we could identify the following proteins: 1) precursor of the heat shock protein gp96; 2) cytosolic calcium-dependent phospholipase A2 (group IVA); 3) $\alpha$-actinin $4 ; 4$ ) heat shock protein $1-\beta$ of $90 \mathrm{kD} ; 5)$ fatty acid (long chain) CoA ligase 3 (known as well as acyl-CoA synthetase 3 ); 6 ) tubulin $\beta 5$; 7) adipophilin; 8 ) $\beta$-actin; 9) $N A D(P)$ dependent steroid dehydrogenase like protein and glyceraldehyde-3phosphate dehydrogenase. Although the lack of washing results in the detection of proteins not typical for LDs such as actin and tubulin, most other proteins were indeed already identified as LDs-associated showing the rational of our approach.

\subsection{TIP47 secondary structure and modifications}

\subsubsection{Database search}

Despite the knowledge of the exact aminoacid composition of the full-length TIP47 protein, almost nothing is known about structural features or about putative posttranslational modifications, no it is known how TIP47 associates with the LD membrane. However, these aspects are of great importance in order to understand how TIP47 is recruited to membrane, whether it has binding partners and how its function is regulated.

The analysis of TIP47 using the PredictProtein site resulted in the prediction of a diffuse globular structure without defined domains, such as membrane spanning domains. The only structural features of TIP47 known so far are putative coiled-coil regions in the $\mathrm{C}$-terminal half of the protein and the $\mathrm{N}$-terminal PAT domain (Figure 4.23.). The latter is of high homology to sequences also found in perilipin and adipophilin (PAT = perilipin, adipophilin, TIP47). The PAT domain of TIP47 containing the residues $22-116$ share $60 \%$ identity with adipophilin and is to $38 \%$ identical and $60 \%$ similar to the amino-terminal region of human perilipin A (Garcia et al., 2003). 


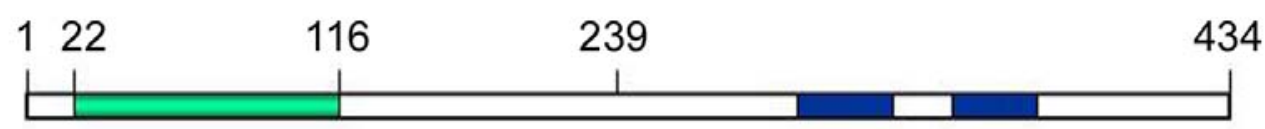

Figure 4.23. Known structural features of TIP47. The protein is thought to contain three main parts the $\mathrm{N}$-terminal PAT-containing domain (1-116, PAT domain is shown in green), a fragment homologous to the adipophilin LD targeting domain (117-239) and the C-terminal half containing putative coiled-coil regions (blue).

We also searched for putative lipid anchors in TIP47 which could mediate interaction of TIP47 with the lipid droplets. According to the Prosite documentation palmitoylation can be predicted for Cys (Ser, Thr, Lys) in any position. TIP47 possesses 3 Cys residues $(39,60,341)$ and each of them could be potentially palmitoylated. No C-terminal GPI-anchor or N-terminal myristoylation sites were found in the full length TIP47 a/a sequence by Prosite, big-PI Predictor v. 1.5 program and MYR Prediction Server.

The Prosite search resulted in the prediction of a number of potential Protein kinase C (PKC) and Casein kinase II (CK2) phosphorylation sites (16 Ser and 7 Thr in total) throughout the entire TIP47 sequence. By using the NetPhos 2.0 Server, 18 Ser, 7 Thr and 3 Tyr potential phosphorylation sites were predicted.

Two SUMO attachment sites with high probability (K195, score 0.6722 and K283, score 0.7556 ) were predicted by SUMOplot\&trade Prediction Development by Abgent. The functional significance of this prediction is however totally unclear and awaits experimental proof.

\subsubsection{TIP47 is a phosphoprotein}

Phosphorylation is the most frequent protein modification regulating the function and intracellular translocation of proteins. However, it is not known so far whether TIP47 is a phosphoprotein. Since TIP47 contains many predicted phosphorylation sites and since its recruitment to the LD and/or its recycling into the cytosol, as well as its function might be regulated by phosphorylation, we analysed the potential incorporation of [32P]-phosphate into TIP47. In a preliminary experiment, HeLa cells were labeled with [Y32P]-ATP under three conditions: under low fatty acid concentration ( $5 \% \mathrm{dFCS})$, high ( $5 \% \mathrm{dFCS}+600 \mu \mathrm{M}$ oleic acid) fatty acid conditions; 
and thirdly cells were preincubated with $600 \mu \mathrm{M}$ oleic acid in standard medium and labeled in DMEM supplemented with $5 \%$ dFCS.

Immunoprecipitation of TIP47 was followed by SDS-PAGE of the precipitated material and autoradiography. Unfortunately, the autoradiograph was quite dirty with high background. However, a band corresponding to the molecular weight of TIP47 was labeled in all three samples with varying intensities. ${ }^{32} \mathrm{P}$-labeling increased by $66 \%$ in cells incubated in the presence of $600 \mu \mathrm{M}$ oleic acid as compared to cells labeled under low FAs conditions, and decreased by $38 \%$ in cells preincubated with $600 \mu \mathrm{M}$ oleic acid and labeled under low FAs conditions (Figure 4.24).

This very preliminary finding suggests that human TIP47 is phosphorylated in vivo and that its phosphorylation status may depend on the availability of fatty acids for the cells. This result however has to be confirmed by additional experiments and is just considered as a first hint.

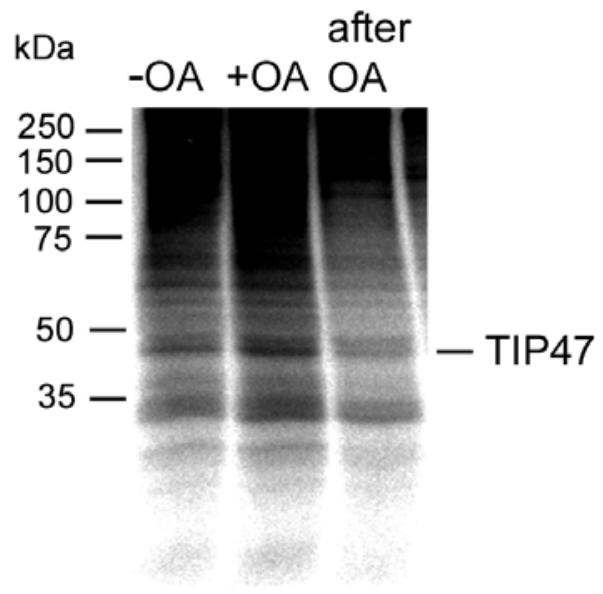

Figure 4.24. Phosphorylation of TIP47 in vivo. TIP47 was immunorecipitated from HeLa cells labeled with ${ }^{32} \mathrm{P}$ under low fatty acid conditions (- OA), under high fatty acid concentration in the medium (+ $\mathrm{OA}$ ) and under low fatty acid conditions after preincubation with $600 \mu \mathrm{M}$ oleic acid. TIP47 phosphorylation was stimulated by high concentrations of oleic acid (OA) and may reflect a desensibilisation to the stimulation by the addition of fresh DMEM with $5 \%$ dFCS.

\subsection{The LD targeting determinats of TIP47}

The targeting to the LD membrane of proteins that belong to the PAT-family is unclear. To exploit this important aspect of TIP47, the protein was arbitrary divided into 3 parts (Figure 4.23.): 
1) An N-terminal region including the PAT domain (residues 1-116);

2) A sequence homologous to the ADRP-LD recruiting region (McManaman et al., 2003) (residues 117-239);

3) A C-terminal part including two putative coil-coiled regions (residues 240-434). According to the division of the protein, 5 HA-tagged variants of TIP47 were cloned, each containing large deletions on $\mathrm{N}$ - and/or C-termini (Figure 4.25.).

HA-TIP47

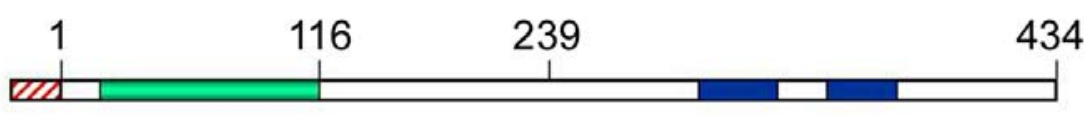

HA-TIP47 1 1-116

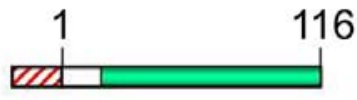

HA-TIP47 $1-239$

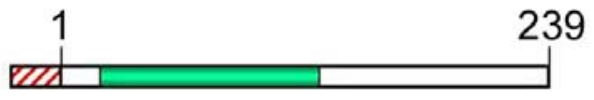

HA-TIP47 $117-239$

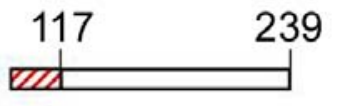

117

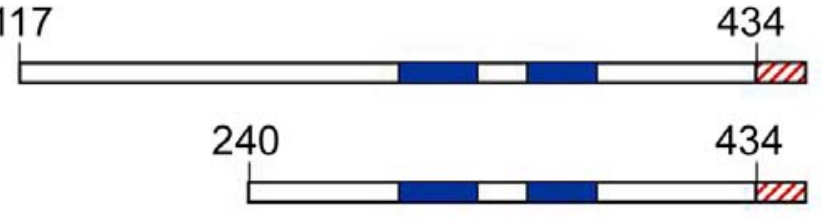

HA-TIP47 $117-434$

HA-TIP47 $240-434$

Figure 4.25. Schematic representation of tagged TIP47 variants. The colours used to indicate domains within TIP47 are the same as in Figure 4.23, HA-tag is shown in red. The lower 5 bars represent the HA-tagged TIP47 containing large deletions in the C-terminal part (HA-TIP47 1-116, HATIP47 ${ }_{1-239}$ ), in the N-terminal region (HA-TIP47 ${ }_{117-434}, \mathrm{HA}-\mathrm{TIP} 47_{240-434}$ ) and a variant in which only the middle part was left (HA-TIP47 $117-239$ ). Five TIP47 truncation variants were tagged on the amino- or carboxy-terminus, as indicated in the figure.

All five TIP47 variants as well as the tagged full length protein were expressed in HeLa cells and their distribution was analysed by indirect immunofluorescence using anti-HA-tag specific antibodies after overnight incubation of the transfected cells in the presence of 300-600 $\mu \mathrm{M}$ oleic acid (Figure 4.26.). Only the full length HA-TIP47 and HA-TIP47 $1-239$ were associated with LDs in all of the expressing cells, while HATIP47 $117-434$ localised to LDs in a small number of cells ( 5\%). All other variants showed a diffuse distribution.

This analysis is therefore suggesting that the targeting of TIP47 to LDs requires the $\mathrm{N}$-terminal half of the protein, while the C-terminal part is not involved. It is however 
important to note, that such large deletions may severely alter the structure of the protein, thereby affecting the recruitment to LD membrane. Thus further studies using much smaller deletions will be needed to narrow down the exact sequence that mediates LD membrane association.
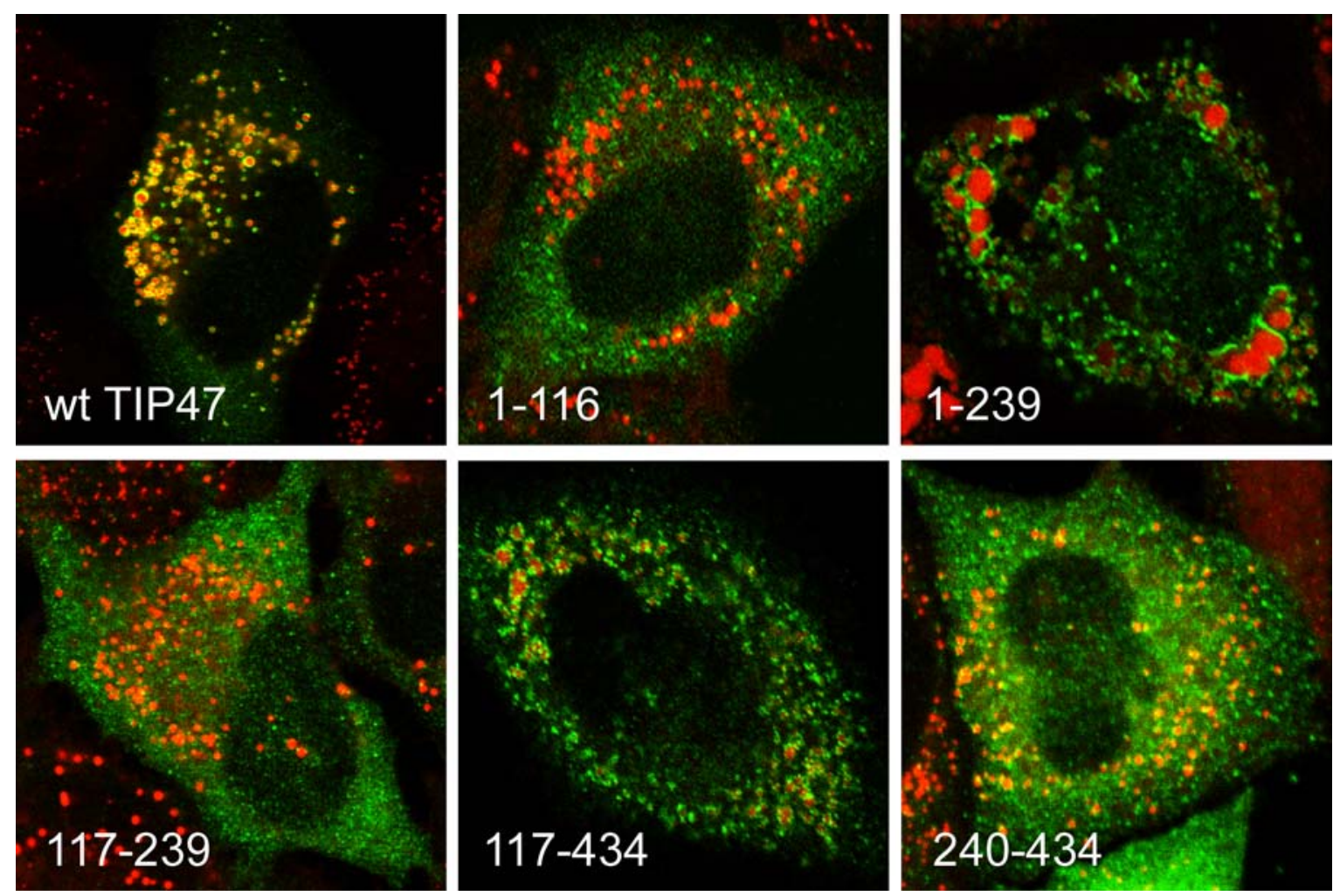

Figure 4.26. Distribution of TIP47 fragments in HeLa cells. One day after transient transfection with full length HA-tagged TIP47 or with one of the 5 TIP47 truncation variants, HeLa cells were incubated $12 \mathrm{~h}$ in presence of 300 or $600 \mu \mathrm{M}$ oleic acid and processed for immunofluorescence. Three of the tagged proteins, HA-TIP47 1 1116, HA-TIP47 $117-239$ and HA-TIP47 $240-434$ exhibited a diffuse localization. HA-TIP47 ${ }_{117-434}$ was associated with LDs in only about $5 \%$ of the transfected cells, while full length HA-TIP47 and HA-TIP47 1-239 were recruited to the LDs in all transfected cells. The HA-tag is shown in green, neutral lipids are shown in red. 


\section{Discussion}

\subsection{TIP47 does not participate in MPRs trafficking}

TIP47 is supposed to be one of the candidate proteins that functions as a cargo selection devise for retrieval of MPRs from late endosomes. The idea is based on seven publications of the Suzanne R. Pfeffer's group. They analysed different aspects of TIP47 including its binding to MPRs and to the small GTP-ase rab9 (Barbero et al., 2002; Carroll et al., 2001; Diaz and Pfeffer, 1998; Hanna et al., 2002; Krise et al., 2000; Orsel et al., 2000; Sincock et al., 2003). Originally, TIP47 was found in a yeast two-hybrid system as a protein interacting with MPRs cytoplasmic tails as a bait (Diaz and Pfeffer, 1998). The work of the group suggested that TIP47

1) binds cytoplasmic domains of both MPRs in vitro;

2) colocalizes with MPR300 (immunofluorescence analysis);

3) is required for recycling of MPR46 from endosomes to the TGN in an in vitro transport assay;

4) is important for MPR300 stability in cells, where TIP47 expression was suppressed by antisence oligonucleotides;

5) specifically binds the FW motif in the MPR46 cytoplasmic domain. This motif was characterized by others as a lysosomal avoidance signal (Schweizer et al., 1997).

However, several recent publications have provided evidence for another function of TIP47 apart from a role in MPRs recycling. Wolins et al. (2001) showed that TIP47 does not colocalize with MPR46, but decorates the surface of LDs in HeLa cells. The results were questioned by S.R.Pfeffer but confirmed by two other independent groups (Miura et al., 2002; Than et al., 2003). In more detail, Pfeffer and colleagues (Barbero et al., 2001) claimed that TIP47 was visible on LDs due to low specificity and cross-reactivity of the antiserum used by Wolins et al., (2001). The authors insisted that the antiserum recognizes two additional proteins, adipophilin and an unknown protein in HeLa cell, and that usage of affinity purified antiserum against TIP47 does not result in staining of LDs in indirect immunofluorescence. This assertion is however very questionable since Wolins et al. (2001) tested the 
antiserum used and found no cross-reactivity in western blotting and immunoprecipitation.

In the next publication on the issue, GFP-TIP47 fusion protein was found to concentrate around LDs in vitro and proposed to be a multifunctional protein, which has a potential to participate in both MPRs transport and LD formation (Miura et al., 2002). However, more convincing data were published just recently by Than et al. 2003, who detected TIP47 on LDs using a different antibody, which specificity to TIP47 was unequivocally confirmed by immunoprecipitation followed with MALDI-MS. Another issue of uncertainty was the question of how TIP47 is recruited to membranous organelles from the cytoplasm. It was postulated that TIP47 is recruited to membranes due to its binding to MPRs cytoplasmic domains and that the interaction appears to be hydrophilic, as TIP47 could be released from membranes by $1 \mathrm{M} \mathrm{KCl}$ (Diaz and Pfeffer, 1998). However, the same authors later showed that TIP47 binding to both MPRs is of hydrophobic nature (Diaz and Pfeffer, 1998; Orsel et al., 2000).

In the beginning of this work we wanted to use TIP47 as a marker protein of a special population of endosomes that are part of the MPR trafficking routes. However, the first simple intracellular localization of TIP47 via immunofluorescence raised doubts according to the published data.

To avoid any accusation in producing artefacts due to low specificity or crossreactivity of any anti-TIP47 antibodies used, we tested our antibodies in Western blotting and immunoprecipitation. Further, we cloned TIP47 fusion proteins with HAand His- tags. We found out that both antibodies (donated by S.R. Pfeffer or purchased from Progen) recognize a single protein in HeLa cell lysates in Western blotting and immunoprecipitation (Figure 4.1.) and give a signal perfectly overlapping with anti-HA or His antibodies when used in immunofluorescence on cells expressing tagged TIP47 (Figure 4.6.).

After having confirmed the specificity of our antibodies against TIP47, we analyzed the distribution of endogenous and tagged TIP47 by means of immunofluorescence and cell fractionation followed by western blot. Both endogenous and tagged TIP47 were indeed found to surround LDs stained with Nile red (Figure 4.3., Figure 4.26.). TIP47 did not colocalize with both MPRs nor with markers of endosomes and 
lysosomes (Figure 4.3., Figure 4.6.) confirming that TIP47 is a LD associated protein (Wolins et al., 2001; Miura et al., 2002; Than et al., 2003). At the same time about $16 \%$ of total cellular amount of TIP47 were found in association with LDs upon lipid loading and subcellular fractionation (Figure 4.7.). Remarkably, TIP47 does not cofractionates with fractions devoid of LDs in TIP47 RNAi cells (Figure 4.15.), indicating that in these cells it is virtually absent in the cytosol and does not associate with membranous organelles apart from LDs.

In addition when the RNAi cells were analysed with respect to effect on MPRs we found out that MPRs trafficking was not affected at all. MPRs distribution (Figure 4.16.), recycling of anti-MPRs antibodies (Figure 4.17.) and the half life of MPR46 (not shown) were not affected in TIP47 RNAi cells. G.R. Medigeshi in the group of P.Shue could also show that a lysosomal enzyme cathepsin D, dependent in its trafficking on MPRs, was not missorted and that TIP47 is not required for MPR46 recycling in an in vitro transport assay (Medigeshi and Schu, 2003). Moreover, we were also informed by another group that it was not possible to reproduce the in vitro interaction between TIP47 and rab9 (Markus Rudolf, unpublished data).

Taken together, the data presented here as well as published earlier (Wolins et al., 2001; Miura et al., 2002; Than et al., 2003; Medigeshi and Schu, 2003) demonstrate that TIP47 is a constituent of LDs and has no role in MPRs transport.

\subsection{LDs biogenesis}

The formation of LDs in mammalian cells is generally assumed to be the result of the biosynthetic activity of ER localized enzymes and pooling of triacylglycerol or other lipids down to LDs (Murphy, 2001). In contrast to mammalian LDs, which were not extensively studied with respect to their biosynthetic activity and protein composition, yeast LDs are well established biosynthetically active organelles (Murphy, 2001; Sorger and Daum, 2003). However, the number of publications describing the protein composition of mammalian LDs (e.g. (Liu et al., 2003)) and their enzymatic activities (Yu et al., 2000) is growing.

In mammalian cells, LD biogenesis as well as the regulation of its function are only poorly understood. Some models have been proposed, which in many aspects 
remain illusive why I want to point a few important aspects that are related to my current work.

The first question is whether LDs are organelles? Are they indeed surrounded by an individual membrane, or conversely are they in continuity with the ER? The question seems to be simple to answer, however, some of the methods were not correctly used in the past and therefore many studies addressing the aspect of LD organelle identity are of very limited value. Immunofluorescence studies are for example beyond the limit of resolution to allow a clearcut answer. The tubular ER network emerges into almost all areas of the cell and thus almost all other cellular structures are in very close apposition to the ER.

One would immediately try to make use of electron microscopy (EM). However sample preparation for EM is known to cause artefacts such as breakage of small connections between organelles. If therefore an ultrastructural analysis could show the existence of individual LDs, it would be of evidence but better be coroborated by other methodology. In this context it should also be note that LD function as well as biogenesis might possess cell-type specific differences. It could very well be that a hepatocyte which is very active in lipid metabolism possess a highly regulated machinery for LD biogenesis and turnover, while a fibroblast may only contain rudiments of this machinery and in consequence is less well organized with respect to LD organization.

A second question that is of general importance concerns LD biogenesis as well as the organelle lipid metabolism. If one assume that LD are organelles well separated from the ER, can they still incorporate triacylglycerol and/or can they still import precursors for triacylglycerol synthesis? If not, this would mean that only when still in connection with the ER fatty acids are metabolized to triacylglycerol which gets incorporated into the growing LD. If the answer is yes, this would mean that an individual LD must be able to transport triacylglycerol from the cytosol across its single leaflet membrane into the interior. Similarly a special mechanism has to be present in case triacylglycerol precursors are imported into the LD. In consequence of the last idea LDs would also contain machinery to metabolize such precursors as well as those enzymes to eventually break down the stored LD contents such as triacylglycerol or cholesterol esters. Within this context one has to be aware of the 
fact that it is also unclear in which way highly hydrophobic molecules such as triacylglycerol are transported through the cytosol.

In with context my own observation might be of interest. When analyzed by immunofluorescence, up to $4 \mathrm{~h}$ incubation with oleic acid, the exposed cells contained LDs fully surrounded by TIP47 but devoid of Nile red staining (Figure 4.10., Figure 4.11). The yellow spectral emission of Nile Red is indicative of high hydrophobisity (Greenspan et al., 1985b). In conclusion more mature LDs containing high concentrations of neutral lipid and exhibiting bright staining of Nile red differs from "younger" LDs with respect of their hydrophobisity. One interpretation of this finding could be as follows: when extracellular concentration of free unesterified fatty acids is high, LDs appear to accumulate first substrates or intermediates of triacylglycerol synthesis, which are later converted into TAG. If this is true, the phospholipid monolayer of nascent LDs should be highly destabilized by the contents. This is why LD might need a number of scaffolding/structural proteins and, probably, chaperons to maintain the integrity of their surface. Such proteins may include members of the family of PAT proteins such as TIP47.

\subsection{The function of TIP47 in LD biogenesis}

We have unequivocally shown that endogenous (Figure 4.3., Figure 4.7) as well as tagged TIP47 (Figure 4.26.) is a constituent of LDs and confirmed that its recruitment to the organelles is induced by administration of fatty acids, in particular oleic acid (Wolins et al., 2001). Later analysis of the time course of recruitment demonstrated that TIP47 decorates nascent LDs rather than mature ones which are indicated by Nile red staining (Figure 4.10.). TIP47 recruitment occurs early upon administration of fatty acids and, under our experimental conditions, achieves its maximum before LDs mature (summarized in Figure 5.1.). We then analysed different aspects of LD biogenesis or processes preceding it with respect to a putative function of TIP47. 


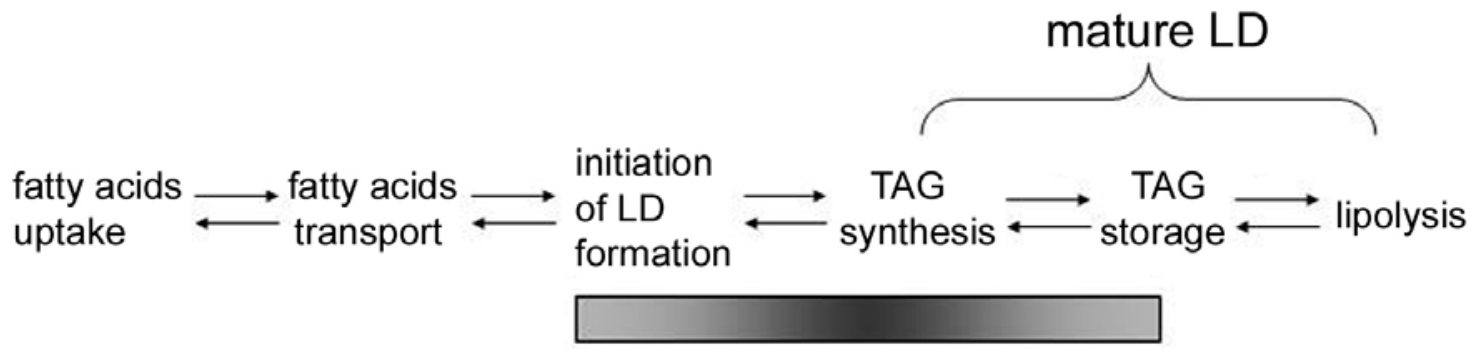

TIP47

Figure 5.1. Shown is the maximal recruitment of TIP47 (indicated by the colour intensity) within life cycle of LDs.

We found that more than $90 \%$ depletion of TIP47 by RNAi does not affect the efficiency of long chain fatty acids uptake by HeLa cells (Figure 4.19.) and has no major effect on both the protein and the phospholipid composition of LDs ( Figure 4.21., Figure 4.22.). However, it significantly impairs the morphology of the LD population (Figure 4.18.) and decreases the amount of fatty acids incorporated into LDs by $50 \%$ (Figure 4.20.). We propose that TIP47 may function as a scaffolding protein important to ensue rapid formation of LDs upon starvation (discussed in 4.1.6.) and to maintain the integrity of nascent LD. The suggestion could explain the alterations in TIP47 RNAi cells. The LDs of the affected cells became larger and fewer since the lowered level of TIP47 may coat only some LDs and more fatty acids are transported to the same droplet to compensate for the lack of TIP47 (surface to volume ratio is lower). However, the total amount of stored material still was reduced when cells were incubated in presence of oleic acid for $12 \mathrm{~h}$. Under our experimental conditions the remaining small amount of TIP47 might be still sufficient to continuously promote the formation of new LDs, which would explain why the RNAi cells could accumulate roughly normal amounts of lipid after longer periods of exposure. Therefore, the effect of TIP47 suppression is most dramatic early after exposure of cells to fatty acids.

A TIP47 function in stabilization of the phospholipid monolayer of nascent LDs would be of special significance if amphipathic precursors of triacylglycerol, such as fatty acids and diacylglycerol, would, indeed, accumulate in the "young" LDs (see 5.2.). In this scenario, lack of TIP47 would inhibit LD growth and maturation because, e.g. 
diacylglycerol, due to its physicochemical properties, would destabilize the ordered phospholipid structures (Goni and Alonso, 1999).

Alternatively, TIP47 may function as a fatty acid transport protein, directly participating in delivery and incorporation of fatty acids into the LDs. The hypothesis is supported by the fact that TIP47 is abundant in the cytoplasm and that its recruitment to LD is efficiently induced by addition of fatty acids. However it is not clear yet whether its association with LD surface is transient and whether the protein is continuously recycling to and from the organelles. Similar proposals were made for adipophilin, the most closely related protein to TIP47. The authors suggested that adipophilin is a saturable fatty acid transport protein (Gao and Serrero, 1999; Serrero et al., 2000) or that it is important for cholesterol targeting to LD (Frolov et al., 2000), nevertheless, the function of adipophilin is not entirely clear yet (see 1.1.3.1.).

It was assumed earlier that TIP47 might play a role in LDs aggregation while it has coiled-coil structures, which could enable its dimerization or even oligomerization (Than et al., 2003), however, it could not explain the phenotype of the TIP47 RNAi cells.

\subsection{TIP47 recruitment to the LDs}

Proteins studied by now with respect of their association with LDs can be divided into four groups:

1) plant and viral proteins containing long hydrophobic stretches embedded into the phospholipid monolayer and, probably, the core of LDs, and requiring the "proline knot" motif as in oleosins and caleosins (Murphy, 2001). Hepatitis C virus (HCV) and GB virus-B core proteins (Hope et al., 2002) are examples;

2) proteins containing both long hydrophobic and palmitoylated sequences (caveolins) (van Deurs et al., 2003);

3) proteins recruited to LD surface via a docking protein (e.g. hormone sensitive lipase (Syu and Saltiel, 1999));

4) proteins containing neither long hydrophobic sequences nor known motifs, but demanding long targeting regions with a certain degree of redundancy and, probably, intact secondary structure (perilipin A (Garcia et al., 2003) and 
adipophilin (McManaman et al., 2003; Nakamura and Fujimoto, 2003; TargettAdams et al., 2003)).

The knowledge of the mechanisms of TIP47 recruitment to LDs as well as involved targeting determinants may give us an opportunity to better understand the function of the protein on LD. We could show that the N-terminal half of TIP47 is sufficient for LD targeting (Figure 4.26.).

In this context it is interesting to note that the interaction of perilipins, adipophilin and TIP47 with the LD surface was found to be hydrophobic, none of the proteins can be dislodged from the membranes by alkali carbonate (Londos et al., 1999; Wolins et al., 2001).

Furthermore, the distribution of our TIP47 deletion variants is remarkably similar to that observed for adipophilin mutants, suggesting that a conserved sequence is required. large deletions of either $\mathrm{C}$ - or N-termini of TIP47 yield proteins with intracellular distribution similar to that of human adipophilin truncation variants, e.g. HA-TIP471-239 (on LD surface in all transfected cells) and HA-TIP47 240-434 (diffuse in $100 \%$ transfected cells) (Figure 4.26.) have nearly the same distribution as GFP$\mathrm{ADRP}_{1-221}$ (mainly in association with LDs) and GFP-ADRP ${ }_{221-437}$ (diffuse in most of transfected cells), respectively (Targett-Adams et al., 2003) despite the difference in the tags introduced and other experimental conditions.

The mode of interaction of the PAT proteins with LDs is not entirely clear, however, it is worth to mention several proposals made.

In one of the early publications, it was suggested that human adipophilin could be anchored to membranes via covalently bound lipid moieties, while there were some evidence that the protein is acylated between residues 93-109 with 5-6 mol of acyl groups per 1 mol of protein (Heid et al., 1996). But the proposal seems unlikely, since others could show that the region of the protein is not required for association with LDs (Nakamura and Fujimoto, 2003; Targett-Adams et al., 2003) (Figure 5.2). 


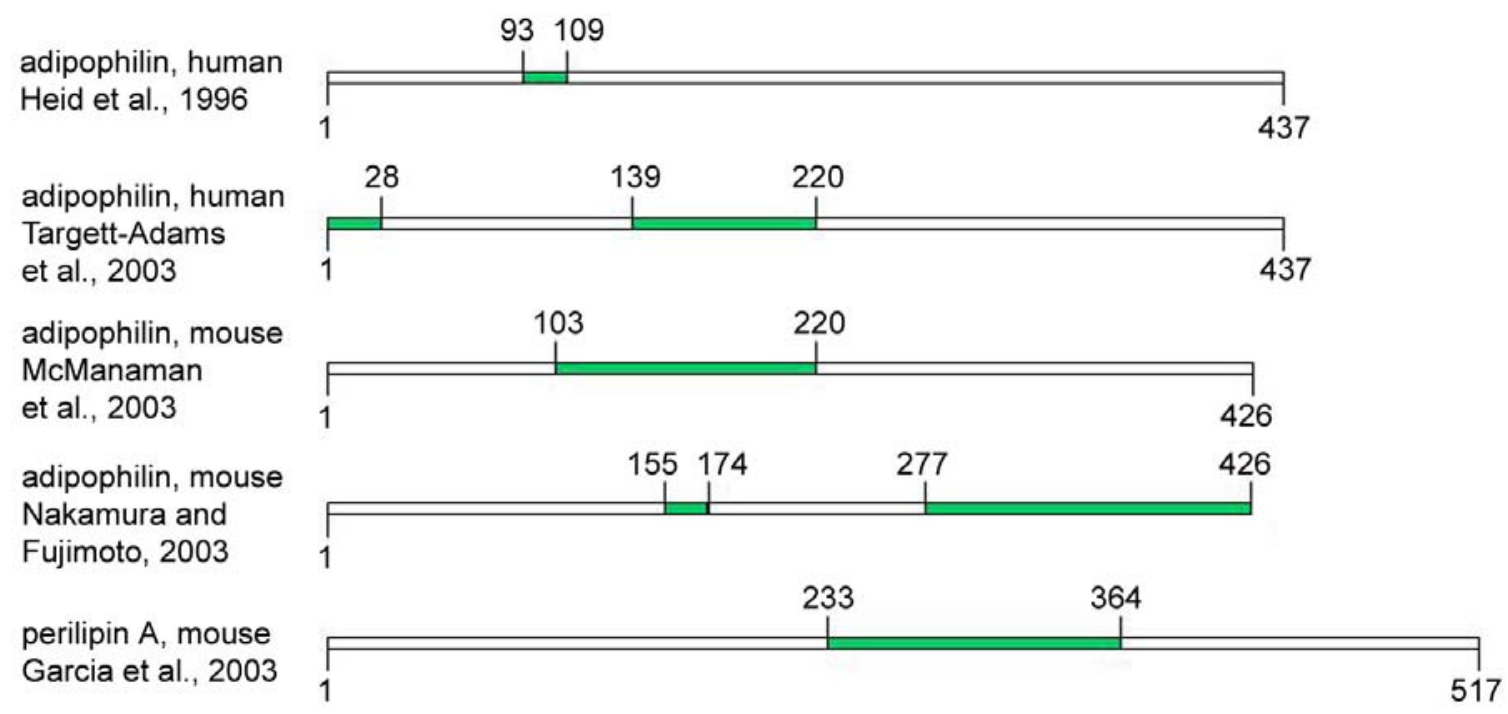

Figure 5.2. LD targeting regions of adipophilin and perilipin A. Regions found to be most important for LD targeting are shown in green.

Lu et al. (Lu et al., 2001) hypothesized that the N-terminal high homology PAT domain is essential for function and/or subcellular localization, perhaps as a targeting moiety to the surface of LD. However, till now no proof for importance of the PAT domain or its common function in the protein family was obtained. Remarkably, the PAT domain turned out to be of no special significance for LD targeting of different PAT family members: human (Heid et al., 1996; Targett-Adams et al., 2003) or mouse (McManaman et al., 2003; Nakamura and Fujimoto, 2003) adipophilin, perilipin A (Garcia et al., 2003) and it is not sufficient for LD association of TIP47 according to our data (Figure 4.26).

Most recently it was suggested that the key feature which determines the interaction of adipophilin with LDs are amphipathic a-helices (McManaman et al., 2003) found also in apolipoproteins (Segrest et al., 1992). Furthermore, it would be interesting to investigate whether TIP47 and other PAT proteins behave similar to $\alpha$-synuclein, the Parkinson's disease protein which is also able to associate with LDs (Cole et al., 2002). $\alpha$-synuclein is unfolded in solution (Weinreb et al., 1996) but adopt a structure with strong $\alpha$-helical character in the presence of membranes containing acidic phospholipids (Davidson et al., 1998; Jo et al., 2000). Interestingly, a-synuclein binding to membranes can be stabilized by cross-linking, which results in isolation of dimers and trimers (Cole et al., 2002). 
In conclusion further work is required to characterized the exact sequence(s)/motif that target PAT family proteins to LDs. In addition it awaits further poof whether the association with LDs also requires the help of a certain post-translational modification. 


\section{$6 \quad$ Future directions}

The work performed here is the basis for the future investigation of TIP47 function and early stages of LD biogenesis. We are going to analyse early stages of LD formation using fluorescently labeled fatty acids and cholesterol as well as GFP-tagged TIP47;

to test whether TIP47 is directly involved in incorporation of fatty acids into LDs and whether its function on LDs is tissue specific;

to search for putative TIP47 partners using various chemical cross-linkers;

to check whether TIP47 phosphorylation/dephosphorylation cycles regulate recruitment of the protein to LDs;

to further analyse LD targeting determinants of TIP47;

to create a single adipophilin and a double adipophilin/TIP47 RNAi cell lines to get an insite into ADRP function and to elucidate whether adipophilin could compensate for lack of TIP47 and to study the effect of RNAi of TIP47 and/or adipophilin in specialized cell types such as adipocytes. 


\section{$7 \quad$ Literature}

1. Almahbobi, G., and Hall, P. F. (1990). The role of intermediate filaments in adrenal steroidogenesis., Journal of Cell Science 97 ( Pt 4), 679-87.

2. Almahbobi, G., Williams, L. J., and Hall, P. F. (1992). Attachment of steroidogenic lipid droplets to intermediate filaments in adrenal cells., Journal of Cell Science 101 ( Pt 2), 383-93.

3. Artelt, P., Morelle, C., Ausmeier, M., Fitzek, M., and Hauser, H. (1988). Vectors for efficient expression in mammalian fibroblastoid, myeloid and lymphoid cells via transfection or infection., Gene 68, 213-9.

4. Athenstaedt, K., Zweytick, D., Jandrositz, A., Kohlwein, S. D., and Daum, G. (1999). Identification and characterization of major lipid particle proteins of the yeast Saccharomyces cerevisiae., Journal of Bacteriology 181, 6441-8.

5. Barbero, P., Bittova, L., and Pfeffer, S. R. (2002). Visualization of Rab9-mediated vesicle transport from endosomes to the trans-Golgi in living cells., The Journal of Cell Biology 156, 511-8.

6. Barbero, P., Buell, E., Zulley, S., and Pfeffer, S. R. (2001). TIP47 Is Not a Component of Lipid Droplets, J Biol Chem 276, 24348-24351.

7. Blanchette-Mackie, E. J., Dwyer, N. K., Barber, T., Coxey, R. A., Takeda, T., Rondinone, C. M., Theodorakis, J. L., Greenberg, A. S., and Londos, C. (1995). Perilipin is located on the surface layer of intracellular lipid droplets in adipocytes., Journal of Lipid Research 36, 1211-26.

8. Boord, J. B., Fazio, S., and Linton, M. F. (2002). Cytoplasmic fatty acid-binding proteins: emerging roles in metabolism and atherosclerosis., Current Opinion in Lipidology 13, 141-7.

9. Bozza, P. T., Yu, W., Penrose, J. F., Morgan, E. S., Dvorak, A. M., and Weller, P. F. (1997). Eosinophil lipid bodies: specific, inducible intracellular sites for enhanced eicosanoid formation., The Journal of Experimental Medicine 186, 909-20.

10.Brasaemle, D. L., Barber, T., Kimmel, A. R., and Londos, C. (1997a). Posttranslational regulation of perilipin expression. Stabilization by stored intracellular neutral lipids., The Journal of Biological Chemistry 272, 9378-87.

11.Brasaemle, D. L., Barber, T., Wolins, N. E., Serrero, G., Blanchette Mackie, E. J., and Londos, C. (1997b). Adipose differentiation-related protein is an ubiquitously expressed lipid storage droplet-associated protein., Journal of Lipid Research 38, 2249-63.

12. Brasaemle, D. L., Levin, D. M., Adler-Wailes, D. C., and Londos, C. (2000a). The lipolytic stimulation of 3T3-L1 adipocytes promotes the translocation of hormone-sensitive lipase to the surfaces of lipid storage droplets., Biochimica Et Biophysica Acta 1483, 251-62.

13. Brasaemle, D. L., Rubin, B., Harten, I. A., Gruia-Gray, J., Kimmel, A. R., and Londos, C. (2000b). Perilipin A increases triacylglycerol storage by 
decreasing the rate of triacylglycerol hydrolysis., The Journal of Biological Chemistry 275, 38486-93.

14. Brummelkamp, T. R., Bernards, R., and Agami, R. (2002). A system for stable expression of short interfering RNAs in mammalian cells., Science 296, $550-$ 3.

15. Buhman, K. K., Chen, H. C., and Farese, R. V. (2001). The enzymes of neutral lipid synthesis., The Journal of Biological Chemistry 276, 40369-72.

16. Cao, Y., Pearman, A. T., Zimmerman, G. A., Mclntyre, T. M., and Prescott, S. M. (2000). Intracellular unesterified arachidonic acid signals apoptosis., Proceedings of the National Academy of Sciences of the United States of America 97, 11280-5.

17. Carroll, K. S., Hanna, J., Simon, I., Krise, J., Barbero, P., and Pfeffer, S. R. (2001). Role of Rab9 GTPase in facilitating receptor recruitment by TIP47., Science 292, 1373-6.

18. Cases, S., Smith, S. J., Zheng, Y. W., Myers, H. M., Lear, S. R., Sande, E., Novak, S., Collins, C., Welch, C. B., Lusis, A. J., et al. (1998). Identification of a gene encoding an acyl CoA:diacylglycerol acyltransferase, a key enzyme in triacylglycerol synthesis., Proceedings of the National Academy of Sciences of the United States of America 95, 13018-23.

19. Chanarin, I., Patel, A., Slavin, G., Wills, E. J., Andrews, T. M., and Stewart, G. (1975). Neutral-lipid storage disease: a new disorder of lipid metabolism., British Medical Journal 1, 553-5.

20. Chao, F. F., Stiers, D. L., and Ontko, J. A. (1986). Hepatocellular triglyceride synthesis and transfer to lipid droplets and nascent very low density lipoproteins., Journal of Lipid Research 27, 1174-81.

21. Christiansen, K., and Jensen, P. K. (1972). Membrane-bound lipid particles from beef heart. Chemical composition and structure., Biochimica Et Biophysica Acta 260, 449-59.

22. Claussen, M., Buergisser, D., Schuller, A. G., Matzner, U., and Braulke, T. (1995). Regulation of insulin-like growth factor (IGF)-binding protein-6 and mannose 6-phosphate/IGF-II receptor expression in IGF-IL-overexpressing NIH 3T3 cells., Molecular Endocrinology (Baltimore, Md) 9, 902-12.

23. Clifford, G. M., Londos, C., Kraemer, F. B., Vernon, R. G., and Yeaman, S. J. (2000). Translocation of hormone-sensitive lipase and perilipin upon lipolytic stimulation of rat adipocytes., The Journal of Biological Chemistry 275, 50115.

24. Cole, N. B., Murphy, D. D., Grider, T., Rueter, S., Brasaemle, D., and Nussbaum, R. L. (2002). Lipid droplet binding and oligomerization properties of the Parkinson's disease protein alpha-synuclein., The Journal of Biological Chemistry 277, 6344-52.

25. Cousin, B., Casteilla, L., Dani, C., Muzzin, P., Revelli, J. P., and Penicaud, L. (1993). Adipose tissues from various anatomical sites are characterized by different patterns of gene expression and regulation., The Biochemical Journal 292 ( Pt 3), 873-6. 
26. Davidson, W. S., Jonas, A., Clayton, D. F., and George, J. M. (1998). Stabilization of alpha-synuclein secondary structure upon binding to synthetic membranes., The Journal of Biological Chemistry 273, 9443-9.

27. Di Mauro, S., Trevisan, C., and Hays, A. (1980). Disorders of lipid metabolism in muscle., Muscle \& Nerve 3, 369-88.

28. Diaz, E., and Pfeffer, S. R. (1998). TIP47: a cargo selection device for mannose 6-phosphate receptor trafficking., Cell 93, 433-43.

29. DiDonato, D., and Brasaemle, D. L. (2003). Fixation methods for the study of lipid droplets by immunofluorescence microscopy., The Journal of Histochemistry and Cytochemistry : Official Journal of the Histochemistry Society 51, 77380.

30.Dodds, P. F. (1995). Xenobiotic lipids: the inclusion of xenobiotic compounds in pathways of lipid biosynthesis., Progress in Lipid Research 34, $219-47$.

31.Dvorak, A. M., Dvorak, H. F., Peters, S. P., Shulman, E. S., MacGlashan, D. W., Pyne, K., Harvey, V. S., Galli, S. J., and Lichtenstein, L. M. (1983). Lipid bodies: cytoplasmic organelles important to arachidonate metabolism in macrophages and mast cells., Journal of Immunology (Baltimore, Md : 1950) 131, 2965-76.

32.Dvorak, A. M., Morgan, E. S., Tzizik, D. M., and Weller, P. F. (1994). Prostaglandin endoperoxide synthase (cyclooxygenase): ultrastructural localization to nonmembrane-bound cytoplasmic lipid bodies in human eosinophils and $3 \mathrm{~T} 3$ fibroblasts., International Archives of Allergy and Immunology 105, 245-50.

33.Egan, J. J., Greenberg, A. S., Chang, M. K., and Londos, C. (1990). Control of endogenous phosphorylation of the major CAMP-dependent protein kinase substrate in adipocytes by insulin and beta-adrenergic stimulation., The Journal of Biological Chemistry 265, 18769-75.

34.Egan, J. J., Greenberg, A. S., Chang, M. K., Wek, S. A., Moos, M. C., and Londos, C. (1992). Mechanism of hormone-stimulated lipolysis in adipocytes: translocation of hormone-sensitive lipase to the lipid storage droplet., Proceedings of the National Academy of Sciences of the United States of America 89, 8537-41.

35. Elbashir, S. M., Harborth, J., Lendeckel, W., Yalcin, A., Weber, K., and Tuschl, T. (2001). Duplexes of 21-nucleotide RNAs mediate RNA interference in cultured mammalian cells., Nature 411, 494-8.

36. Feng, B., and Tabas, I. (2002). ABCA1-mediated cholesterol efflux is defective in free cholesterol-loaded macrophages. Mechanism involves enhanced ABCA1 degradation in a process requiring full NPC1 activity., The Journal of Biological Chemistry 277, 43271-80.

37.Fong, T. H., and Wang, S. M. (1997). Dissection of the signaling mechanism for capsule detachment of lipid droplets in rat adrenocortical cells., Journal of Cellular Biochemistry 65, 67-74.

38. Fong, T. H., Wang, S. M., and Lin, H. S. (1996). Immunocytochemical demonstration of a lipid droplet-specific capsule in cultured Leydig cells of the golden hamsters., Journal of Cellular Biochemistry 63, 366-73. 
39. Fong, T. H., Yang, C. C., Greenberg, A. S., and Wang, S. M. (2002). Immunocytochemical studies on lipid droplet-surface proteins in adrenal cells., Journal of Cellular Biochemistry 86, 432-9.

40. Franke, W. W., Hergt, M., and Grund, C. (1987). Rearrangement of the vimentin cytoskeleton during adipose conversion: formation of an intermediate filament cage around lipid globules., Cell 49, 131-41.

41.Frolov, A., Petrescu, A., Atshaves, B. P., So, P. T., Gratton, E., Serrero, G., and Schroeder, F. (2000). High density lipoprotein-mediated cholesterol uptake and targeting to lipid droplets in intact L-cell fibroblasts. A single- and multiphoton fluorescence approach., The Journal of Biological Chemistry 275, 12769-80.

42. Fujimoto, T., Kogo, H., Ishiguro, K., Tauchi, K., and Nomura, R. (2001). Caveolin2 is targeted to lipid droplets, a new "membrane domain" in the cell., The Journal of Cell Biology 152, 1079-85.

43. Fujino, T., Kang, M. J., Suzuki, H., lijima, H., and Yamamoto, T. (1996). Molecular characterization and expression of rat acyl-CoA synthetase 3., The Journal of Biological Chemistry 271, 16748-52.

44. Gao, J., and Serrero, G. (1999). Adipose differentiation related protein (ADRP) expressed in transfected COS-7 cells selectively stimulates long chain fatty acid uptake., The Journal of Biological Chemistry 274, 16825-30.

45. Gao, J., Ye, H., and Serrero, G. (2000). Stimulation of adipose differentiation related protein (ADRP) expression in adipocyte precursors by long-chain fatty acids., Journal of Cellular Physiology 182, 297-302.

46. Garcia, A., Sekowski, A., Subramanian, V., and Brasaemle, D. L. (2003). The central domain is required to target and anchor perilipin A to lipid droplets., The Journal of Biological Chemistry 278, 625-35.

47. Ghosal, D., Shappell, N. W., and Keenan, T. W. (1994). Endoplasmic reticulum lumenal proteins of rat mammary gland. Potential involvement in lipid droplet assembly during lactation., Biochimica Et Biophysica Acta 1200, 175-81.

48. Gibbons, G. F., Islam, K., and Pease, R. J. (2000). Mobilisation of triacylglycerol stores., Biochimica Et Biophysica Acta 1483, 37-57.

49. Gibbons, G. F., and Wiggins, D. (1995). Intracellular triacylglycerol lipase: its role in the assembly of hepatic very-low-density lipoprotein (VLDL)., Advances in Enzyme Regulation 35, 179-98.

50. Goldstein, J. L., Ho, Y. K., Basu, S. K., and Brown, M. S. (1979). Binding site on macrophages that mediates uptake and degradation of acetylated low density lipoprotein, producing massive cholesterol deposition., Proceedings of the National Academy of Sciences of the United States of America 76, 333-7.

51. Goni, F. M., and Alonso, A. (1999). Structure and functional properties of diacylglycerols in membranes., Progress in Lipid Research 38, 1-48.

52. Greenberg, A. S., Egan, J. J., Wek, S. A., Garty, N. B., Blanchette-Mackie, E. J., and Londos, C. (1991). Perilipin, a major hormonally regulated adipocytespecific phosphoprotein associated with the periphery of lipid storage droplets., The Journal of Biological Chemistry 266, 11341-6. 
53. Greenberg, A. S., Egan, J. J., Wek, S. A., Moos, M. C., Londos, C., and Kimmel, A. R. (1993). Isolation of cDNAs for perilipins A and B: sequence and expression of lipid droplet-associated proteins of adipocytes., Proceedings of the National Academy of Sciences of the United States of America 90, 12035-9.

54. Greenspan, P., Mayer, E. P., and Fowler, S. D. (1985a). Nile red: a selective fluorescent stain for intracellular lipid droplets., The Journal of Cell Biology 100, 965-73.

55. Greenspan, and Fowler, S.D. (1985b). Spectrofluorometric studies of the lipid probe, Nile red., The Journal of Lipid Research 26, 781-9.

56. Guo, W., Choi, J. K., Kirkland, J. L., Corkey, B. E., and Hamilton, J. A. (2000). Esterification of free fatty acids in adipocytes: a comparison between octanoate and oleate., The Biochemical Journal 349, 463-71.

57. Hanna, J., Carroll, K., and Pfeffer, S. R. (2002). Identification of residues in TIP47 essential for Rab9 binding., Proceedings of the National Academy of Sciences of the United States of America 99, 7450-4.

58. Hay, J. C., Hirling, H., and Scheller, R. H. (1996). Mammalian vesicle trafficking proteins of the endoplasmic reticulum and Golgi apparatus., The Journal of Biological Chemistry 271, 5671-9.

59. Heid, H. W., Moll, R., Schwetlick, I., Rackwitz, H. R., and Keenan, T. W. (1998). Adipophilin is a specific marker of lipid accumulation in diverse cell types and diseases., Cell and Tissue Research 294, 309-21.

60. Heid, H. W., Schnolzer, M., and Keenan, T. W. (1996). Adipocyte differentiationrelated protein is secreted into milk as a constituent of milk lipid globule membrane., The Biochemical Journal 320 ( Pt 3), 1025-30.

61. Hope, R. G., Murphy, D. J., and McLauchlan, J. (2002). The domains required to direct core proteins of hepatitis $C$ virus and $G B$ virus-B to lipid droplets share common features with plant oleosin proteins., The Journal of Biological Chemistry 277, 4261-70.

62. Hulbert, W. C., Guppy, M., Murphy, B., and Hochachka, P. W. (1979). Metabolic sources of heat and power in tuna muscles. I. Muscle fine structure., The Journal of Experimental Biology 82, 289-301.

63. Igal, R. A., and Coleman, R. A. (1996). Acylglycerol recycling from triacylglycerol to phospholipid, not lipase activity, is defective in neutral lipid storage disease fibroblasts., The Journal of Biological Chemistry 271, 16644-51.

64. Igal, R. A., and Coleman, R. A. (1998). Neutral lipid storage disease: a genetic disorder with abnormalities in the regulation of phospholipid metabolism., Journal of Lipid Research 39, 31-43.

65. Imamura, M., Inoguchi, T., Ikuyama, S., Taniguchi, S., Kobayashi, K., Nakashima, N., and Nawata, H. (2002). ADRP stimulates lipid accumulation and lipid droplet formation in murine fibroblasts., American Journal of Physiology Endocrinology and Metabolism 283, E775-83.

66. Jiang, H. P., Harris, S. E., and Serrero, G. (1992). Molecular cloning of a differentiation-related mRNA in the adipogenic cell line 1246., Cell Growth \& 
Differentiation : the Molecular Biology Journal of the American Association For Cancer Research 3, 21-30.

67. Jo, E., McLaurin, J., Yip, C. M., St George-Hyslop, P., and Fraser, P. E. (2000). alpha-Synuclein membrane interactions and lipid specificity., The Journal of Biological Chemistry 275, 34328-34.

68. Kamisaka, Y., Mishra, S., and Nakahara, T. (1997). Purification and characterization of diacylglycerol acyltransferase from the lipid body fraction of an oleaginous fungus., 121, 1107-14.

69. Klumperman, J., Hille, A., Veenendaal, T., Oorschot, V., Stoorvogel, W., von Figura, K., and Geuze, H. J. (1993). Differences in the endosomal distributions of the two mannose 6-phosphate receptors., The Journal of Cell Biology 121, 997-1010.

70. Krise, J. P., Sincock, P. M., Orsel, J. G., and Pfeffer, S. R. (2000). Quantitative analysis of TIP47-receptor cytoplasmic domain interactions: implications for endosome-to-trans Golgi network trafficking., Journal of Biological Chemistry 275, 25188-93.

71. Leber, R., Landl, K., Zinser, E., Ahorn, H., Spok, A., Kohlwein, S. D., Turnowsky, F., and Daum, G. (1998). Dual localization of squalene epoxidase, Erg1p, in yeast reflects a relationship between the endoplasmic reticulum and lipid particles., Molecular Biology of the Cell 9, 375-86.

72. Leber, R., Zinser, E., Zellnig, G., Paltauf, F., and Daum, G. (1994). Characterization of lipid particles of the yeast, Saccharomyces cerevisiae., Yeast (Chichester, West Sussex) 10, 1421-8.

73. Lefevre, C., Jobard, F., Caux, F., Bouadjar, B., Karaduman, A., Heilig, R., Lakhdar, H., Wollenberg, A., Verret, J. L., Weissenbach, J., et al. (2001). Mutations in CGI-58, the gene encoding a new protein of the esterase/lipase/thioesterase subfamily, in Chanarin-Dorfman syndrome., American Journal of Human Genetics 69, 1002-12.

74. Lehner, R., Cui, Z., and Vance, D. E. (1999). Subcellullar localization, developmental expression and characterization of a liver triacylglycerol hydrolase., The Biochemical Journal 338 ( Pt 3), 761-8.

75. Lehner, R., and Kuksis, A. (1996). Biosynthesis of triacylglycerols., Progress in Lipid Research 35, 169-201.

76. Lieber, J. G., and Evans, R. M. (1996). Disruption of the vimentin intermediate filament system during adipose conversion of 3T3-L1 cells inhibits lipid droplet accumulation., Journal of Cell Science 109 ( Pt 13), 3047-58.

77. Liu, P., Ying, Y., Zhao, Y., Mundy, D. I., Zhu, M., and Anderson, R. G. W. (2003). $\mathrm{CHO} \mathrm{K} 2$ cell lipid droplets appear to be metabolic organelles involved in membrane traffic, J Biol Chem, M311945200.

78. Londos, C., Brasaemle, D. L., Schultz, C. J., Segrest, J. P., and Kimmel, A. R. (1999). Perilipins, ADRP, and other proteins that associate with intracellular neutral lipid droplets in animal cells., Seminars in Cell \& Developmental Biology 10, 51-8.

79.Lu, X., Gruia_Gray, J., Copeland, N. G., Gilbert, D. J., Jenkins, N. A., Londos, C., and Kimmel, A. R. (2001). The murine perilipin gene: the lipid droplet- 
associated perilipins derive from tissue-specific, mRNA splice variants and define a gene family of ancient origin., Mammalian Genome : Official Journal of the International Mammalian Genome Society 12, 741-9.

80.Luiken, J. J., Schaap, F. G., van_Nieuwenhoven, F. A., van_der_Vusse, G. J., Bonen, A., and Glatz, J. F. (1999). Cellular fatty acid transport in heart and skeletal muscle as facilitated by proteins., Lipids 34 Suppl, S169-75.

81. Mallard, F., Antony, C., Tenza, D., Salamero, J., Goud, B., and Johannes, L. (1998). Direct pathway from early/recycling endosomes to the Golgi apparatus revealed through the study of shiga toxin B-fragment transport., The Journal of Cell Biology 143, 973-90.

82. Martinez-Botas, J., Anderson, J. B., Tessier, D., Lapillonne, A., Chang, B. H., Quast, M. J., Gorenstein, D., Chen, K. H., and Chan, L. (2000). Absence of perilipin results in leanness and reverses obesity in $\operatorname{Lepr}(\mathrm{db} / \mathrm{db})$ mice., Nature Genetics 26, 474-9.

83. Marz, K. E., Lauer, J. M., and Hanson, P. I. (2003). Defining the SNARE complex binding surface of alpha-SNAP: implications for snare complex disassembly., The Journal of Biological Chemistry 278, 27000-8.

84. Mather, I. H. (2000). A review and proposed nomenclature for major proteins of the milk-fat globule membrane., Journal of Dairy Science 83, 203-47.

85. May, C., Preisig_Muller, R., Hohne, M., Gnau, P., and Kindl, H. (1998). A phospholipase A2 is transiently synthesized during seed germination and localized to lipid bodies., Biochimica Et Biophysica Acta 1393, 267-76.

86. McManaman, J. L., Zabaronick, W., Schaack, J., and Orlicky, D. J. (2003). Lipid droplet targeting domains of adipophilin., Journal of Lipid Research 44, 66873.

87. Medigeshi, G. R., and Schu, P. (2003). Characterization of the in vitro retrograde transport of MPR46., 4, 802-11.

88. Meyer, C., Zizioli, D., Lausmann, S., Eskelinen, E. L., Hamann, J., Saftig, P., von_Figura, K., and Schu, P. (2000). mu1A-adaptin-deficient mice: lethality, loss of AP-1 binding and rerouting of mannose 6-phosphate receptors., The Embo Journal 19, 2193-203.

89. Milla, P., Athenstaedt, K., Viola, F., Oliaro_Bosso, S., Kohlwein, S. D., Daum, G., and Balliano, G. (2002). Yeast oxidosqualene cyclase (Erg7p) is a major component of lipid particles., The Journal of Biological Chemistry 277, 240612.

90. Miura, S., Gan, J. W., Brzostowski, J., Parisi, M. J., Schultz, C. J., Londos, C., Oliver, B., and Kimmel, A. R. (2002). Functional conservation for lipid storage droplet association among Perilipin, ADRP, and TIP47 (PAT)-related proteins in mammals, Drosophila, and Dictyostelium., The Journal of Biological Chemistry 277, 32253-7.

91. Moskalenko, S., Henry, D. O., Rosse, C., Mirey, G., Camonis, J. H., and White, M. A. (2002). The exocyst is a Ral effector complex., Nature Cell Biology 4 , 66-72.

92. Murphy, D. J. (2001). The biogenesis and functions of lipid bodies in animals, plants and microorganisms., Progress in Lipid Research 40, 325-438. 
93. Nakamura, N., and Fujimoto, T. (2003). Adipose differentiation-related protein has two independent domains for targeting to lipid droplets., Biochemical and Biophysical Research Communications 306, 333-8.

94. Noll, F., May, C., and Kindl, H. (2000). Phospholipid monolayer of plant lipid bodies attacked by phospholipase A2 shows $80 \mathrm{~nm}$ holes analyzed by atomic force microscopy., Biophysical Chemistry 86, 29-35.

95. Ohashi, M., Mizushima, N., Kabeya, Y., and Yoshimori, T. (2003). Localization of mammalian $\mathrm{NAD}(\mathrm{P}) \mathrm{H}$ steroid dehydrogenase-like protein on lipid droplets., The Journal of Biological Chemistry 278, 36819-29.

96. Orsel, J. G., Sincock, P. M., Krise, J. P., and Pfeffer, S. R. (2000). Recognition of the $300-k D a$ mannose 6 -phosphate receptor cytoplasmic domain by $47-k D a$ tail-interacting protein., Proceedings of the National Academy of Sciences of the United States of America 97, 9047-51.

97. Ostermeyer, A. G., Paci, J. M., Zeng, Y., Lublin, D. M., Munro, S., and Brown, D. A. (2001). Accumulation of caveolin in the endoplasmic reticulum redirects the protein to lipid storage droplets., The Journal of Cell Biology 152, 1071-8.

98. Osuga, J., Ishibashi, S., Oka, T., Yagyu, H., Tozawa, R., Fujimoto, A., Shionoiri, F., Yahagi, N., Kraemer, F. B., Tsutsumi, O., and Yamada, N. (2000). Targeted disruption of hormone-sensitive lipase results in male sterility and adipocyte hypertrophy, but not in obesity., Proceedings of the National Academy of Sciences of the United States of America 97, 787-92.

99. Pillai, M. G., Certik, M., Nakahara, T., and Kamisaka, Y. (1998). Characterization of triacylglycerol biosynthesis in subcellular fractions of an oleaginous fungus, Mortierella ramanniana var. angulispora., Biochimica Et Biophysica Acta 1393, 128-36.

100. Pol, A., Luetterforst, R., Lindsay, M., Heino, S., Ikonen, E., and Parton, R. G. (2001). A caveolin dominant negative mutant associates with lipid bodies and induces intracellular cholesterol imbalance., The Journal of Cell Biology 152, 1057-70.

101. Prattes, S., Horl, G., Hammer, A., Blaschitz, A., Graier, W. F., Sattler, W., Zechner, R., and Steyrer, E. (2000). Intracellular distribution and mobilization of unesterified cholesterol in adipocytes: triglyceride droplets are surrounded by cholesterol-rich ER-like surface layer structures., Journal of Cell Science 113 (Pt 17), 2977-89.

102. Rohrer, J., Schweizer, A., Johnson, K. F., and Kornfeld, S. (1995). A determinant in the cytoplasmic tail of the cation-dependent mannose 6phosphate receptor prevents trafficking to lysosomes., The Journal of Cell Biology 130, 1297-306.

103. Ross, R. (1995). Cell biology of atherosclerosis., Annual Review of Physiology 57, 791-804.

104. Saltiel, A. R. (2000). Another hormone-sensitive triglyceride lipase in fat cells?, Proceedings of the National Academy of Sciences of the United States of America 97, 535-7.

105. Saltiel, A. R., and Kahn, C. R. (2001). Insulin signalling and the regulation of glucose and lipid metabolism., Nature 414, 799-806. 
106. Scherer, P. E., Bickel, P. E., Kotler, M., and Lodish, H. F. (1998). Cloning of cell-specific secreted and surface proteins by subtractive antibody screening., Nature Biotechnology 16, 581-6.

107. Schweizer, A., Kornfeld, S., and Rohrer, J. (1997). Proper sorting of the cation-dependent mannose 6-phosphate receptor in endosomes depends on a pair of aromatic amino acids in its cytoplasmic tail., Proceedings of the National Academy of Sciences of the United States of America 94, 14471-6.

108. Segrest, J. P., Jones, M. K., De Loof, H., Brouillette, C. G., Venkatachalapathi, Y. V., and Anantharamaiah, G. M. (1992). The amphipathic helix in the exchangeable apolipoproteins: a review of secondary structure and function., Journal of Lipid Research 33, 141-66.

109. Serrero, G., Frolov, A., Schroeder, F., Tanaka, K., and Gelhaar, L. (2000). Adipose differentiation related protein: expression, purification of recombinant protein in Escherichia coli and characterization of its fatty acid binding properties., Biochimica Et Biophysica Acta 1488, 245-54.

110. Servetnick, D. A., Brasaemle, D. L., Gruia Gray, J., Kimmel, A. R., Wolff, J., and Londos, C. (1995). Perilipins are associated with cholesteryl ester droplets in steroidogenic adrenal cortical and Leydig cells., The Journal of Biological Chemistry 270, 16970-3.

111. Sincock, P. M., Ganley, I. G., Krise, J. P., Diederichs, S., Sivars, U., O Connor, B., Ding, L., and Pfeffer, S. R. (2003). Self-Assembly Is Important for TIP47 Function in Mannose 6-Phosphate Receptor Transport., 4, 18-25.

112. Small, D. M. (1988). George Lyman Duff memorial lecture. Progression and regression of atherosclerotic lesions. Insights from lipid physical biochemistry., Arteriosclerosis (Dallas, Tex) 8, 103-29.

113. Soldati, T., Rancano, C., Geissler, H., and Pfeffer, S. R. (1995). Rab7 and Rab9 are recruited onto late endosomes by biochemically distinguishable processes., Journal of Biological Chemistry 270, 25541-8.

114. Sorger, D., and Daum, G. (2002). Synthesis of triacylglycerols by the acylcoenzyme A:diacyl-glycerol acyltransferase Dga1p in lipid particles of the yeast Saccharomyces cerevisiae., Journal of Bacteriology 184, 519-24.

115. Sorger, D., and Daum, G. (2003). Triacylglycerol biosynthesis in yeast., Applied Microbiology and Biotechnology 61, 289-99.

116. Souza, S. C., de Vargas, L. M., Yamamoto, M. T., Lien, P., Franciosa, M. D., Moss, L. G., and Greenberg, A. S. (1998). Overexpression of perilipin A and $B$ blocks the ability of tumor necrosis factor alpha to increase lipolysis in 3T3-L1 adipocytes., The Journal of Biological Chemistry 273, 24665-9.

117. Stahl, A., Evans, J. G., Pattel, S., Hirsch, D., and Lodish, H. F. (2002). Insulin causes fatty acid transport protein translocation and enhanced fatty acid uptake in adipocytes., 2, 477-88.

118. Storch, S., and Braulke, T. (2001). Multiple C-terminal motifs of the 46-kDa mannose 6-phosphate receptor tail contribute to efficient binding of medium chains of AP-2 and AP-3., The Journal of Biological Chemistry 276, 4298303. 
119. Stremmel, W., Pohl, L., Ring, A., and Herrmann, T. (2001). A new concept of cellular uptake and intracellular trafficking of long-chain fatty acids., Lipids $36,981-9$.

120. Syu, L. J., and Saltiel, A. R. (1999). Lipotransin: a novel docking protein for hormone-sensitive lipase., Molecular Cell 4, 109-15.

121. Tansey, J. T., Huml, A. M., Vogt, R., Davis, K. E., Jones, J. M., Fraser, K. A., Brasaemle, D. L., Kimmel, A. R., and Londos, C. (2003). Functional studies on native and mutated forms of perilipins. A role in protein kinase Amediated lipolysis of triacylglycerols., The Journal of Biological Chemistry 278, 8401-6.

122. Tansey, J. T., Sztalryd, C., Gruia_Gray, J., Roush, D. L., Zee, J. V., Gavrilova, O., Reitman, M. L., Deng, C. X., Li, C., Kimmel, A. R., and Londos, C. (2001). Perilipin ablation results in a lean mouse with aberrant adipocyte lipolysis, enhanced leptin production, and resistance to diet-induced obesity., Proceedings of the National Academy of Sciences of the United States of America 98, 6494-9.

123. Targett-Adams, P., Chambers, D., Gledhill, S., Hope, R. G., Coy, J. F., Girod, A., and McLauchlan, J. (2003). Live cell analysis and targeting of the lipid droplet-binding adipocyte differentiation-related protein., The Journal of Biological Chemistry 278, 15998-6007.

124. Tauchi-Sato, K., Ozeki, S., Houjou, T., Taguchi, R., and Fujimoto, T. (2002). The surface of lipid droplets is a phospholipid monolayer with a unique Fatty Acid composition., The Journal of Biological Chemistry 277, 44507-12.

125. Than, N. G., Sumegi, B., Bellyei, S., Berki, T., Szekeres, G., Janaky, T., Szigeti, A., Bohn, H., and Than, G. N. (2003). Lipid droplet and milk lipid globule membrane associated placental protein 17b (PP17b) is involved in apoptotic and differentiation processes of human epithelial cervical carcinoma cells., European Journal of Biochemistry 270, 1176-88.

126. Than, N. G., Sumegi, B., Than, G. N., Kispal, G., and Bohn, H. (1998). Cloning and sequence analysis of cDNAs encoding human placental tissue protein 17 (PP17) variants., European Journal of Biochemistry 258, 752-7.

127. Than, N. G., Sumegi, B., Than, G. N., Kispal, G., and Bohn, H. (1999). Is placental tissue protein 17b/TIP47 a new factor in cervical cancer genesis?, Anticancer Research 19, 5255-8.

128. van Deurs, B., Roepstorff, K., Hommelgaard, A. M., and Sandvig, K. (2003). Caveolae: anchored, multifunctional platforms in the lipid ocean., Trends in Cell Biology 13, 92-100.

129. van Meer, G. (2001). Caveolin, cholesterol, and lipid droplets?, The Journal of Cell Biology 152, F29-34.

130. Vance, J. E. (1998). Eukaryotic lipid-biosynthetic enzymes: the same but not the same., Trends in Biochemical Sciences 23, 423-8.

131. Wan, L., Molloy, S. S., Thomas, L., Liu, G., Xiang, Y., Rybak, S. L., and Thomas, G. (1998). PACS-1 defines a novel gene family of cytosolic sorting proteins required for trans-Golgi network localization., Cell 94, 205-16. 
132. Wang, S. M., and Fong, T. H. (1995). A lipid droplet-specific capsule is present in rat adrenal cells: evidence from a monoclonal antibody., Biochemical and Biophysical Research Communications 217, 81-8.

133. Wang, S. M., Fong, T. H., Hsu, S. Y., Chien, C. L., and Wu, J. C. (1997). Reorganization of a novel vimentin-associated protein in 3T3-L1 cells during adipose conversion., Journal of Cellular Biochemistry 67, 84-91.

134. Weinreb, P. H., Zhen, W., Poon, A. W., Conway, K. A., and Lansbury, P. T. (1996). NACP, a protein implicated in Alzheimer's disease and learning, is natively unfolded., Biochemistry 35, 13709-15.

135. Weller, P. F., Bozza, P. T., Yu, W., and Dvorak, A. M. (1999). Cytoplasmic lipid bodies in eosinophils: central roles in eicosanoid generation., International Archives of Allergy and Immunology 118, 450-2.

136. Wiggins, D., and Gibbons, G. F. (1992). The lipolysis/esterification cycle of hepatic triacylglycerol. Its role in the secretion of very-low-density lipoprotein and its response to hormones and sulphonylureas., The Biochemical Journal 284 ( Pt 2), 457-62.

137. Wolins, N. E., Rubin, B., and Brasaemle, D. L. (2001). TIP47 Associates with Lipid Droplets, J Biol Chem 276, 5101-5108.

138. Wolins, N. E., Skinner, J. R., Schoenfish, M. J., Tzekov, A., Bensch, K. G., and Bickel, P. E. (2003). Adipocyte protein S3-12 coats nascent lipid droplets., The Journal of Biological Chemistry 278, 37713-21.

139. Wu, C. C., Howell, K. E., Neville, M. C., Yates, J. R., and McManaman, J. L. (2000). Proteomics reveal a link between the endoplasmic reticulum and lipid secretory mechanisms in mammary epithelial cells., Electrophoresis 21, 3470-82.

140. Yao, P. M., and Tabas, I. (2001). Free cholesterol loading of macrophages is associated with widespread mitochondrial dysfunction and activation of the mitochondrial apoptosis pathway., The Journal of Biological Chemistry 276, 42468-76.

141. Yu, W., Bozza, P. T., Tzizik, D. M., Gray, J. P., Cassara, J., Dvorak, A. M., and Weller, P. F. (1998). Co-compartmentalization of MAP kinases and cytosolic phospholipase A2 at cytoplasmic arachidonate-rich lipid bodies., American Journal of Pathology 152, 759-69.

142. Yu, W., Cassara, J., and Weller, P. F. (2000). Phosphatidylinositide 3-kinase localizes to cytoplasmic lipid bodies in human polymorphonuclear leukocytes and other myeloid-derived cells., Blood 95, 1078-85.

143. Zweytick, D., Athenstaedt, K., and Daum, G. (2000). Intracellular lipid particles of eukaryotic cells., Biochimica Et Biophysica Acta 1469, 101-20. 


\section{Acknowledgement}

I want to express my gratitude to Prof. Dr. Kurt von Figura for giving me the opportunity to work in this institute and his encouragement to perform the study.

I thank Prof. Dr. G. Braus for being my co-referee.

My special thanks go to Dr. Stefan Höning for his amazing patience, constant support and for organizing my work in a proper scientific way. Discussing with him generated many fruitful ideas and I deeply appreciate the time he spend teaching me.

Andrea and Christina thank you very much for your cooperation. Thank you for always being there to answer my questions and to help me in the work.

I am grateful to Kirill Demchenko for the time he spent with my computer and for all the software support without which it would have been impossible to finish this thesis.

I thank Kudzai Mutenda and Nicole Eiselt for performing MS-analysis of my samples.

Thank you Constance, Kira, Vera, Vadim, Rekha, Annegret, Subbulakshmi, Björn, Olaf, Magdalena, and Jens for many lively interactions, the opportunity to discuss our work during the lunchbreak.

I acknowledge gratefully the support of Dr. Peter Schu, Dr. Thomas Dierks Dr. Christian Körner, Dr. Gabriele Fischer von Mollard and Dr. Bernhard Schmidt with reagents and equipments for the experiments.

I thank all my colleagues in the institute with who created a familiar working atmosphere, with whom I picked up my "Deutsch" and who helped me with many day to day to things.

Without my friend, Dr. Meik Dilcher's help during the whole period and until the end in organizing, printing, binding etc etc. not to forget botanical excursions to enlighten my mind, this time would not have been so pleasant. 


\section{Curriculum Vitae}

Name:

Date of Birth:

Place of Birth:

$1982-1988$

$1988-1992$

$1993-1997$

1997-1999

M.Sc Project

Jan.-Mai 2000

June 2000 - present
Bulankina Anna Valentinovna

06.08.1975

Rjazan, Russia

Secondary education in Rjazan, Russia.

Secondary education in Tashkent, Uzbekistan.

Bachelor of Science in Biology, at the Department of Cytology and Histology, St.-Petersburg State University, Russia.

Master of Science in Biology at the Department of Embryology, Diploma with Honors, St.-Petersburg State University, Russia

"The interaction of a new hematopoietic progenitor cells marker Ac133 and retinoblastoma gene product".

At Laboratory of Regulation of Gene Expression, Institute of Cytology of Russian Academy of Sciences, St.-Petersburg, Russia.

Worked as Project Assistant with Dr. Popov B.V, Institute of Cytology of Russian Academy of Sciences, St.-Petersburg, Russia.

Joined the "Zentrum für Biochemie und Molekulare Zell Biologie" (Biochemie II) to pursue doctoral studies under the supervision of Dr. Stefan Höning to work on the research project "TIP47 recruitment to lipid droplets and function on the organelle biogenesis" 